\title{
ADVANCED SPECTRAL METHODS FOR CLIMATIC TIME SERIES
}

M. Ghil, ${ }^{1,2}$ M. R. Allen, ${ }^{3}$ M. D. Dettinger, ${ }^{4}$ K. Ide, ${ }^{1}$

D. Kondrashov, ${ }^{1}$ M. E. Mann, ${ }^{5}$ A. W. Robertson, ${ }^{1,6}$

A. Saunders, ${ }^{1}$ Y. Tian, ${ }^{1,7}$ F. Varadi, ${ }^{1}$ and P. Yiou ${ }^{8}$

R eceived 28 A ugust 2000; revised 3 J uly 2001; accepted 18 September 2001; published 13 September 2002.

[1] The analysis of univariate or multivariate time series provides crucial information to describe, understand, and predict climatic variability. The discovery and implementation of a number of novel methods for extracting useful information from time series has recently revitalized this classical field of study. Considerable progress has also been made in interpreting the information so obtained in terms of dynamical systems theory. In this review we describe the connections between time series analysis and nonlinear dynamics, discuss signal-to-noise enhancement, and present some of the novel methods for spectral analysis. The various steps, as well as the advantages and disadvantages of these methods, are illustrated by their application to an important climatic time series, the Southern O scillation Index. This index captures major features of interannual climate variability and is used extensively in its prediction. R egional and global sea surface temperature data sets are used to illustrate multivariate spectral methods. Open questions and further prospects conclude the review. INDEX TERMS: 1620 Climate dynamics (3309); 3220 Nonlinear dynamics; 4522 EI Niño; 9820 Techniques applicable in three or more fields; KEYWORDS: climate; dynamical systems; EI Niño; prediction; spectral analysis; time series

Citation: M. G hil, M . R . Allen, M . D. Dettinger, K. I de, D. Kondrashov, M. E. M ann, A. W. Robertson, A. Saunders, Y . Tian, F. Varadi, and P. Y iou, A dvanced spectral methods for climatic time series, Rev. Geophys., 40(1), 1003, doi:10.1029/2000R G 000092, 2002.

\section{CONTENTS}

1. Introduction and M otivation

1.1. A nalysis in the time domain versus the spectral domain ....................... 3

1.2. Time series and nonlinear dynamics .... 4

2. Enhancing the Signal-to-Noise $(S / N)$ R atio $\ldots 6$

2.1. M otivation for singular spectrum analysis (SSA)

\footnotetext{
${ }^{1}$ Department of A tmospheric Sciences and Institute of Geophysics and Planetary Physics, U niversity of California, Los A ngeles, Los A ngeles, California, USA.

${ }^{2} \mathrm{~A}$ Iso at $D$ épartement Terre-A tmosphère- $O$ céan and $L$ aboratoire de Météorologie Dynamique, Ecole Normale Supérieure, Paris, France.

${ }^{3}$ Space Science and Technology Department, R utherford A ppleton Laboratory, Chilton, Didcot, England.

${ }^{4}$ U.S. G eological Survey, San Diego, California, U SA.

${ }^{5}$ D epartment of E nvironmental Sciences, U niversity of V irginia, Charlottesville, Virginia, USA .

${ }^{6} \mathrm{~N}$ ow at International R esearch Institute for Climate Prediction (IRI), Palisades, N ew Y ork, U SA .

${ }^{7}$ Now at NA SA Goddard Space Flight Center, Greenbelt, $M$ aryland, U SA .

${ }^{8}$ Laboratoire des Sciences du Climat et de l'E nvironnement, UMR CEA-CNRS, G if-sur-Y vette, France.
}

2.2. Decomposition and reconstruction ..... 7

2.3. M onte Carlo SSA ..................... 11

2.4. M ultiscale SSA and wavelet analysis .... 13

3. Spectral A nalysis M ethods ................... 16

3.1. Generalities and definitions ............ 16

3.2. Classical spectral estimates ............. 17

3.3. M aximum entropy method (M E M ) .... 18

3.4. Multitaper method (M TM) ............ 20

4. M ultivariate M ethods ...................... 25

4.1. Principal oscillation patterns (POPs) ... 26

4.2. M ultichannel SSA (M-SSA) ........... 26

5. Summary and Perspectives ................. 30

5.1. Summary ........................... 30

5.2. I mplications for understanding ......... 32

5.3. I mplications for prediction ............ 33

A ppendix A: SSA, Spatial EOFs, and the Karhunen-Lò̀ve Theorems ...................... 34

A 1. Spatial and temporal EOFs ........... 34

A 2. Complementary windows for M-SSA .... 35

\section{INTRODUCTION AND MOTIVATION}

[2] A time series provides useful information about the physical, biological, or socioeconomic system that produced it. The purpose of time series analysis is to determine some of the system's key properties by quan- 
TABLE 1. Glossary of the Principal Symbols ${ }^{a}$

\begin{tabular}{|c|c|c|c|}
\hline Symbol & Definition & Method & Section \\
\hline$A_{k}(t)$ & $k$ th principal component (PC) of $\{X(t)\}$ & SSA & 2.2 \\
\hline$A_{k}^{b}(t)$ & $k$ th local PC of $\{X(t)\}$ at time $b$ & SSA & 2.4 \\
\hline$A(t), A_{n}$ & continuous- and discrete-time envelope function & M TM & 3.4 \\
\hline $\mathbf{A}^{k}$ & $k$ th multichannel PC & SSA & 4.2 \\
\hline$a$ & dilation factor of $\psi(x) \rightarrow \psi(x / a)$ & WLTS & 2.4 \\
\hline$a_{1}$ & lag-one autoregression coefficient & SSA & 2.3 \\
\hline$\left\{a_{j}\right\}$ & true regression coefficients with order $M$ & & 1 \\
\hline$\left\{\hat{a}_{j}\right\}$ & estimates of $a_{j}$ with order $M^{\prime}$ & MEM & 3.3 \\
\hline$B, \hat{B}\left(f_{0}\right)$ & true and estimated amplitude of oscillation (at frequency $f_{0}$ ) & M TM & 3.4 \\
\hline $\mathbf{B}, \mathbf{B}_{\tau}$ & true and estimated dynamics matrix (with $\operatorname{lag} \tau$ ) & POP & 4.1 \\
\hline$b$ & translation of $\psi(x) \rightarrow \psi(x-b)$ & WLTs & 2.4 \\
\hline$b_{k}(f)$ & weighting function & MTM & 3.4 \\
\hline $\mathrm{C}_{R}$ & covariance of surrogate data for $\{\mathbf{X}(t)\}$ & SSA & 2.3 \\
\hline $\mathbf{C}^{(\mathbf{R})}$ & reduced covariance matrix & SSA & Appendix \\
\hline $\mathbf{C}(\tau)$ & lag-covariance matrix of $\{\mathbf{X}(t)\}$ with $\operatorname{lag} \tau$ & POP & 4.1 \\
\hline${\underset{\sim}{X}}_{X}$ & covariance of $\{\mathbf{X}(t)\}$ in the univariate case & SSA & 2.2 \\
\hline$\tilde{\mathbf{C}}_{\mathbf{X}}$ & grand covariance matrix in the multivariate case & SSA & 4.2 \\
\hline$\underset{\sim}{\mathbf{D}}$ & trajectory matrix of $\{\underset{\sim}{\tilde{\mathbf{X}}}(t)\}$ in the univariate case & & 2.2 \\
\hline$\tilde{\mathbf{D}}$ & trajectory matrix of $\left\{\tilde{\mathbf{X}}_{l}(t)\right\}$ in the multivariate case & SSA & 4.2 \\
\hline$d$ & dimension of underlying attractor & & 2.1 \\
\hline$d \xi(t)$ & white noise vector in continuous time & POP & 4.1 \\
\hline $\mathbf{E}^{k}$ & eigenvector matrix of $\tilde{\mathbf{C}}_{\mathbf{X}}$ or $\tilde{\mathbf{T}}_{\mathbf{X}}$ & SSA & 4.2 \\
\hline$F_{i}$ & right-hand sides of ODES & & 2.1 \\
\hline$F(f)$ & $F$ test ratio & M TM & 3.4 \\
\hline$f$ & frequency & SSA & 2.3 \\
\hline$\left\{f_{k}\right\}$ & discrete sequence of frequencies & BT & 3.2 \\
\hline$f_{N}$ & N yquist frequency & & 2.3 \\
\hline$f_{R}$ & R ayleigh frequency & M TM & 3.4 \\
\hline$f_{0}$ & fixed frequency of pure sinusoid & MTM & 3.4 \\
\hline $\mathbf{G}(\tau)$ & Green's function associated with $\mathbf{B}$ at $\operatorname{lag} \tau$ & POP & 4.1 \\
\hline$\left\{g_{j}\right\}$ & smoothing weights for $\bar{S}_{X}\left(f_{k}\right)$ & BT & 3.2 \\
\hline$I$ & interval for dilations $a$ & WLTs & 2.4 \\
\hline$i$ & time index & SSA & 2.4 \\
\hline$i$ & imaginary unit & BT & 3.4 \\
\hline$K$ & number of tapers & M TM & 3.4 \\
\hline $\mathscr{K}$ & a set of indices used in reconstruction & SSA & 2.2 \\
\hline$L$ & number of channels & POP & 4.1 \\
\hline$M$ & order of autoregression & & 1 \\
\hline$M$ & embedding dimension & & 2.1 \\
\hline$M \Delta t$ & window width & SSA & 2.2 \\
\hline$M_{t}$ & normalization factor for $R_{\mathscr{K}}(t)$ & SSA & 2.2 \\
\hline$M^{\prime}$ & order of MEM & MEM & 3.3 \\
\hline$N$ & length of $\{\underset{\sim}{X}(n \Delta t)\}$ & & 1 \\
\hline$N_{\sim}^{\prime}$ & length of $\{\tilde{\mathbf{X}}(n \Delta t)\}, N^{\prime}=N-M+1$ & & 2.1 \\
\hline$\tilde{N}$ & normalization factor for $\tilde{\mathbf{T}}_{\mathbf{x}}$ & SSA & 4.2 \\
\hline$P(f)$ & cumulative power spectrum & & 3.1 \\
\hline$p$ & integer bandwidth parameter & M TM & 3.4 \\
\hline $\mathbf{Q}$ & lag-zero covariance matrix of $d \xi(t)$ & POP & 4.1 \\
\hline$R_{\mathscr{Y}}(t)$ & reconstructed component (RC) of $\{X(t)\}$ for a set $\mathscr{K}$ & SSA & 2.2 \\
\hline$R_{\mathscr{Y}}^{b}(t)$ & $\mathscr{K}$ th local RC of $\{X(t)\}$ at time $b$ & SSA & 2.4 \\
\hline $\mathbf{R}^{k}$ & $k$ th multichannel $\mathrm{RC}$ & SSA & 4.2 \\
\hline$r$ & lag-one autocorrelation & SSA & 2.3 \\
\hline$S(f)$ & power spectrum of $A R(1)$ process & SSA & 2.3 \\
\hline$S_{0}$ & average of $S(f)$ & SSA & 2.3 \\
\hline${\underset{\sim}{X}}_{X}(f), \hat{S}_{X}(f)$ & true and estimated periodogram & BT & 3.2 \\
\hline$\tilde{S}_{X}(f)=$ & correlogram & BT & 3.2 \\
\hline $\bar{S}_{X}\left(f_{k}\right), \overline{\bar{S}}_{X}(f)$ & direct and indirect estimate of $S_{X}(f)$ & BT & 3.2 \\
\hline$S_{X}(f), \hat{S}_{X}(f)$ & true and estimated (by $\left\{\hat{a}_{j}\right\}$ ) power spectrum & BT & 3.2 \\
\hline$\hat{S}_{k}(f)$ & estimated $k$ th eigenspectrum & M TM & 3.4 \\
\hline$S_{\sim}(f), S_{w}(f)$ & high-resolution and adaptively weighted multitaper spectrum & M TM & 3.4 \\
\hline$\tilde{\mathbf{T}}_{\mathbf{X}}$ & grand block matrix for covariances & SSA & 4.2 \\
\hline$t^{-x}$ & continuous time $(t \in \mathbb{R})$ or discrete time $\left(t \in \mathbb{Z}^{+}\right)$ & & 1 \\
\hline$\Delta t$ & sampling rate & & 1 \\
\hline
\end{tabular}


TABLE 1. (continued)

\begin{tabular}{|c|c|c|c|}
\hline Symbol & Definition & Method & Section \\
\hline$n \Delta t$ & discretely sampled time & & 1 \\
\hline$U_{k}(f)$ & discrete Fourier transform of $w_{k}(t)$ & MTM & 3.4 \\
\hline$W$ & sliding-window length of wavelet & SSA & 2.4 \\
\hline$W_{\psi}(a, b)$ & wavelet transform of $\{X(t)\}$ using $b$-translated and $a$-dilated $\psi((t-b) / a)$ & SSA & 2.4 \\
\hline$W_{\mu}^{\mu}(k)$ & lag window for $\overline{\bar{S}}_{X}(f)$ with smoothing parameter $\mu$ & BT & 3.2 \\
\hline$W_{m}(k)$ & B artlett window for $\overline{\bar{S}}_{X}(f)$ with window length $m$ & BT & 3.2 \\
\hline$w_{k}(t)$ & $k$ th taper & MTM & 3.4 \\
\hline$\hat{X}(t)$ & observed time series & & 1 \\
\hline$X_{n}$ & $X(n \Delta t)$ & & 1 \\
\hline$X^{(p)}$ & $p$ th-order differentiation of $X(t)$ with respect to time $t, d^{p} X / d t^{p}$ & & 1 \\
\hline$\{X(t)\}$ & univariate time series in $t \in \mathbb{R}$ or $t \in \mathbb{Z}^{+}$ & & 1 \\
\hline $\mathbf{X}(t)$ & $M$-dimensional augmented vector of $X(t)$ & & 2.1 \\
\hline$X_{0}$ & mean of $\{\mathbf{X}(t)\}$ & SSA & 2.3 \\
\hline$\underset{\sim}{X}(t)$ & reconstructed $X(t)$ & WLTS & 2.4 \\
\hline$\tilde{X}(t), \tilde{X}(n \Delta t)$ & continuous- and discrete-time reconstructed signal & MTM & 3.4 \\
\hline$\{\underset{\sim}{\mathbf{X}}(t)\}$ & multivariate time series in $t \in \mathbb{R}$ or $\underset{\sim}{t} \in \mathbb{Z}^{+}$ & POP & 4.1 \\
\hline$\left\{\tilde{\mathbf{X}}_{l}\right\}$ & multichannel augmented vector of $\{\tilde{\mathbf{X}}(t)\}$ & SSA & 4.2 \\
\hline$\hat{\hat{Y}}_{k}(f)$ & discrete Fourier transform of $\left\{X(t) w_{k}(t)\right\}$ & MTM & 3.4 \\
\hline$\alpha$ & time-scale ratio & SSA & 2.4 \\
\hline$\eta(t)$ & additive noise & MTM & 3.4 \\
\hline$\theta$ & explained contribution to variance & MTM & 3.4 \\
\hline$\lambda_{k}, \Lambda_{X}$ & $k$ th eigenvalue and eigenvalue matrix of $\mathbf{C}_{X}$ & SSA & 2.2 \\
\hline$\Lambda_{R}$ & projection of $\mathbf{C}_{R}$ onto $\mathbf{E}_{X}$ & SSA & 2.3 \\
\hline$\mu_{k}$ & weight of $k$ th taper & MTM & 3.4 \\
\hline$v$ & number of degrees of freedom of spectrum & MTM & 3.4 \\
\hline$\xi(t)$ & random forcing & & 1 \\
\hline$\rho_{k}, \mathbf{E}_{X}$ & $k$ th eigenvector and eigenvector matrix of $\mathbf{C}_{X}$ & SSA & 2.2 \\
\hline$\sigma^{2}$ & variance of random forcing & & 1 \\
\hline$\tau$ & characteristic delay time & SSA & 2.3 \\
\hline$\phi_{X}(k), \hat{\phi}_{X}(k)$ & true and estimated lag $k$ autocorrelation function of $X(t)$ & BT & 3.2 \\
\hline$\psi(x)$ & mother wavelet of variable $x$ & WLTS & 2.4 \\
\hline$\psi$ & unexplained contribution to variance & M T M & 3.4 \\
\hline
\end{tabular}

aM ethod and section number correspond to where the symbol appears first or is used differently from previous sections.

tifying certain features of the time series. These properties can then help understand the system's behavior and predict its future.

[3] To illustrate the ideas and methods reviewed here, we shall turn to one of the best known climatic time series. This time series is made up of monthly values of the Southern Oscillation Index (SOI). It will be introduced in section 2.2 and is shown in Figure 2 there.

[4] At this point we merely note that physical processes usually operate in continuous time. Most measurements, though, are done and recorded in discrete time. Thus the SOI time series, as well as most climatic and other geophysical time series, are available in discrete time.

\subsection{Analysis in the Time Domain Versus the Spectral Domain}

[5] Two basic approaches to time series analysis are associated with the time domain or the spectral domain. We present them at first in the linear context in which the physical sciences have operated for most of the last two centuries. In this context the physical system can be described by a linear ordinary differential equation
(ODE), or a system of such equations, subject to additive random forcing.

[6] It goes well beyond the scope of this review paper to introduce the concepts of random variables, stochastic processes, and stochastic differential equations. We refer the interested reader to Feller $[1968,1971]$ for the former two concepts and to Arnold [1974] and Schuss [1980] for the latter. M any of the standard books on classical spectral methods that are cited in sections 3.1 and 3.2 also contain good elementary introductions to stochastic processes in discrete and, sometimes, continuous time.

[7] We concentrate here on time series in discrete time and consider therefore first the simple case of a scalar, linear ordinary difference equation with random forcing,

$$
X(t+1)=\sum_{j=1}^{M} a_{j} X(t-M+j)+\sigma \xi(t) .
$$

Its constant coefficients $a_{j}$ determine the solutions $X(t)$ at discrete times $t=0,1, \ldots, n, \ldots . \operatorname{In}(1)$ the random forcing $\xi(t)$ is assumed to be white in time, i.e., uncor- 
related from $t$ to $t+1$, and Gaussian at each $t$, with constant variance equal to unity. In the Yule [1927] and Walker [1931] method for the time domain approach, one computes the coefficients $a_{j}$ and the variance $\sigma^{2}$ from a realization of $X$ having length $N,\{X(t): 1 \leq t \leq$ $N\}$.

[8] This method is discussed further in section 3.3 below, where (1) is treated as an autoregressive (AR) process of order $M$, which we denote by $\mathrm{AR}(M)$. The notation used in the present paper is summarized in Table 1. The table lists the main symbols in al phabetical order and indicates the section where each symbol is introduced first. This facilitates the comparison between the methods we review, since the literature of each method tends to use its own notation.

[9] The spectral domain approach is motivated by the observation that the most regular, and hence predictable, behavior of a time series is to be periodic. This approach then proceeds to determine the periodic components embedded in the time series by computing the associated periods, amplitudes, and phases, in this order.

[10] The classical implementation of the spectral domain approach is based on the Bochner-K hinchin-W iener theorem [Box and Jenkins, 1970], which states that the lag autocorrelation function of a time series and its spectral density are Fourier transforms of each other. Hannan's [1960] introduction to this approach and its implementation excels by its brevity and clarity; the so-called Blackman-Tukey implementation is presented in section 3.2 below. We shall use here both the more mathematical term of spectral density and the term of power spectrum, often encountered in the scientific and engineering literature, according to whether the context is more theoretical or more applied.

[11] The remainder of this review is organized as follows. Section 2 deals mainly with signal-to-noise (S/N) ratio enhancement and introduces singular spectrum analysis (SSA) as an important and flexible tool for this enhancement. Connections between SSA and empirical orthogonal functions (EOFs) are outlined in Appendix A. Statistical tests for the reliability of SSA results are also discussed in this section, along with connections to wavelet analysis.

[12] In section 3 we present, in succession, three methods of spectral analysis: Fourier transform based, maximum entropy, and multitaper. B oth sections 2 and 3 use the SOI time series for the purposes of illustrating the methods "in action." In section 4 the multivariate extensions of the maximum entropy method and of single-channel SSA are introduced, and a few additional applications are mentioned or illustrated. The review concludes with a section on open questions, from the point of view of both the methodology and its applications.

\subsection{Time Series and Nonlinear Dynamics}

[13] Before proceeding with the technical details, we give in this section a quick perspective on the "nonlinear revolution" in time series analysis. I rregularity in observed time series had traditionally been attributed to the above mentioned random "pumping" of a linear system by infinitely many (independent) degrees of freedom (D OF). In the 1960s and 1970s the scientific community found out that much of this irregularity could be generated by the nonlinear interaction of a few DOF [Lorenz, 1963; Smale, 1967; Ruelle and Takens, 1971]. This realization of the possibility of deterministic aperiodicity or "chaos" [Gleick, 1987] created quite a stir.

[14] The purpose of this review is to describe briefly some of the implications of this change in outlook for time series analysis, with a special emphasis on climatic time series. $M$ any general aspects of nonlinear time series analysis are reviewed by Drazin and King [1992], Ott et al. [1994], and Abarbanel [1996]. We concentrate here on those aspects that deal with regularities and have proven most useful in studying climatic variability.

[15] A connection between deterministically chaotic time series and the nonlinear dynamics generating them was attempted fairly early in the young history of "chaos theory." The basic idea was to consider specifically a scalar, or univariate, time series with apparently irregular behavior, generated by a deterministic or stochastic system. This time series could be exploited, so the thinking went, in order to ascertain, first, whether the underlying system has a finite number of DOF. An upper bound on this number would imply that the system is deterministic, rather than stochastic, in nature. N ext, we might be able to verify that the observed irregularity arises from the fractal nature of the deterministic system's invariant set, which would yield a fractional, rather than integer, value of this set's dimension. Finally, one could maybe reconstruct the invariant set or even the equations governing the dynamics from the data.

[16] This ambitious program [Packard et al., 1980; Roux et al., 1980; Ruelle, 1981] relied essentially on the method of delays, based in turn on the Whitney [1936] embedding lemma and the Mañé [1981] and Takens [1981] theorems. We first describe an easy connection between a univariate and a multivariate time series.

[17] Let us assume that the univariate time series is the solution of a scalar nonlinear ODE of order $p$,

$$
X^{(p)}=G\left(X^{(p-1)}, \ldots, X\right) .
$$

This scalar higher-dimensional equation is equivalent to the following system of first-order ODEs:

$$
\dot{X}_{i}=F_{i}\left(X_{1}, \ldots, X_{j}, \ldots, X_{p}\right), \quad 1 \leq i, j \leq p ;
$$

here $\dot{X} \equiv d X / d t \equiv X^{(1)}$ and $X^{(p)} \equiv d^{p} X / d t^{p}$. It suffices to write

$X \equiv X_{1}, \dot{X}_{1}=X_{2}, \ldots, \dot{X}_{p-1}=X_{p}, \dot{X}_{p}=G\left(X_{1}, \ldots, X_{p}\right)$,

so that $F_{1}=X_{2}, F_{2}=X_{3}, \ldots, F_{p}=G$. In other words, the successive derivatives of $X(t)$ can be thought of as the components of a vector $\mathbf{X} \equiv\left(X_{1}, \ldots, X_{p}\right)$. The 
E uclidean space $\mathbb{R}^{p}$ in which the vector $\mathbf{X}=\mathbf{X}(t)$ evolves is called the phase space of the first-order system (3). The graph of this evolution in $\mathbb{R}^{p}$ is called an orbit or a trajectory of equation (3).

[18] Let us now change the point of view and consider system (3) for arbitrary right-hand sides $F_{i}(\mathbf{X})$, rather than for the specific $F_{i}$ given by (4). Such an ODE system represents a fairly general description of a differentiable dynamical system in continuous time [ $\mathrm{Ar}$ nold, 1973, 1983]. We are interested at first in the case in which only a single time series $\hat{X}(t)$ is known, where $\hat{X}=$ $X_{i}$ for some fixed component $i=i_{0}$ or $\hat{X}$ is some sufficiently smooth function of all the components $X_{i}$. For the solutions of such a system to be irregular, i.e., other than (asymptotically) steady or periodic, three or more DOF are necessary. Can one then go from (3) to (2) just as easily as in the opposite direction? The answer, in general is no; hence a slightly more sophisticated procedure needs to be applied.

[19] This procedure is called the method of delays, and it tries, in some sense, to imitate the $Y$ ule-Walker inference of (1) from the time series $\{X(t): t=1, \ldots$, $N\}$. First of all, one acknowledges that the data $X(t)$ are typically given at discrete times $t=n \Delta t$ only. N ext, one admits that it is hard to actually get the right-hand sides $F_{i}$; instead, one attempts to reconstruct the invariant set on which the solutions of (3) that satisfy certain constraints lie.

[20] In the case of conservative, $\mathrm{H}$ amiltonian systems [Lichtenberg and Lieberman, 1992], there are typically unique solutions through every point in phase space. The irregularity in the solutions' behavior is associated with the intricate structure of cantori [Wiggins, 1988], complicated sets of folded tori characterized by a given energy of the solutions lying on them. These cantori have, in particular, finite and fractional dimension, being self-similar fractals [Mandelbrot, 1982].

[21] M athematically speaking, however, $\mathrm{H}$ amiltonian systems are structurally unstable [Smale, 1967] in the function space of all differentiable dynamical systems. Physically speaking, on the other hand, "open" systems, in which energy is gained externally and dissipated internally, abound in nature. Therefore climatic time series, as well as most other time series from nature or the laboratory, are more likely to be generated by forced dissipative systems [Lorenz, 1963; Ghil and Childress, 1987, chapter 5]. The invariant sets associated with irregularity here are "strange attractors" [Ruelle and Takens, 1971], toward which all solutions tend asymptotically; that is, long-term irregular behavior in such systems is associated with these attractors. These objects are also fractal, although rigorous proofs to this effect have been much harder to give than in the case of Hamiltonian cantori [Guckenheimer and Holmes, 1983; Lasota and Mackey, 1994].

[22] Mañé [1981], Ruelle [1981], and Takens [1981] had the idea, developed further by Sauer et al. [1991], that a single observed time series $\hat{X}(t)$ (where $\hat{X}=X_{i_{0}}$ or, more generally, $\left.\hat{X}=\phi\left(X_{1}(t), \ldots, X_{p}(t)\right)\right)$ could be used to reconstruct the attractor of a forced dissipative system. The basis for this reconstruction idea is essentially the fact that the solution that generates $X(t)$ covers the attractor densely; that is, as time increases, this solution will pass arbitrarily close to any point on the attractor. Time series observed in the natural environment, however, have finite length and sampling rate, as well as significant measurement noise.

[23] The embedding idea has been applied therefore most successfully to time series generated numerically or by laboratory experiments in which sufficiently long series could be obtained and noise was controlled better than in nature. Broomhead and King [1986a], for instance, successfully applied SSA to the reconstruction of the Lorenz [1963] attractor. A s we shall see, for climate and other geophysical time series, it might be possible to attain a more modest goal: to describe merely a "skeleton" of the attractor that is formed by a few robust periodic orbits.

[24] In the climate context, Lorenz [1969] had already pointed out a major stumbling block for applying the attractor reconstruction idea to large-scale atmospheric motions. While using more classical statistical methods, he showed that the recurrence time of sufficiently good analogs for weather maps was of the order of hundreds of years, at the spatial resolution of the observational network then available for the Northern $\mathrm{Hemisphere.}$

[25] The next best target for demonstrating from an observed time series the deterministic cause of its irregularity was to show that the presumed system's attractor had a finite and fractional dimension. $V$ arious dimensions, metric and topological, can be defined [Kaplan and Yorke, 1979; Farmer et al., 1983]; among them, the one that became the most popular, since easiest to compute, was the correlation dimension [Grassberger and Procaccia, 1983]. In several applications, its computation proved rather reliable and hence useful. Climatic time series, however, tended again to be rather too short and noisy for comfort (see, for instance, Ruelle [1990] and Ghil et al. [1991] for a review of this controversial topic).

[26] A more robust connection between classical spectral analysis and nonlinear dynamics seems to be provided by the concept of "ghost limit cycles." The road to chaos [Eckmann, 1981] proceeds from stable equilibria, or fixed points, through stable periodic solutions, or limit cycles, and on through quasiperiodic solutions lying on tori, to strange attractors. The fixed points and limit cycles are road posts on this highway from the simple to the complex. That is, even after having lost their stability to successively more complex and realistic solutions, these simple attractors still play a role in the observed spatial patterns and the time series generated by the system.

[27] A "ghost fixed point" is a fixed point that has become unstable in one or a few directions in phase space. Still, the system's trajectories will linger near it for 
(a)

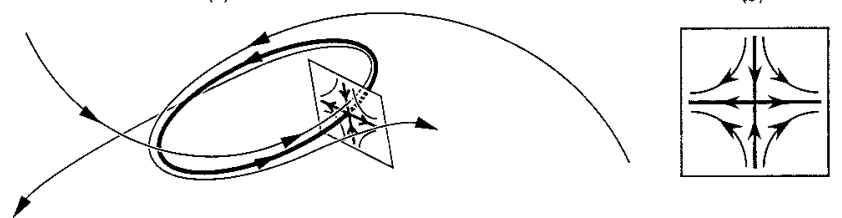

Figure 1. Schematic diagram of a ghost limit cycle. Figure 1a is a perspective sketch of the limit cycle (bold curve) in a three-dimensional E uclidean space. Light solid curves indicate two distinct trajectories that approach the limit cycle in a direction in which it is stable and leave its neighborhood in an unstable direction. Figure $1 \mathrm{~b}$ is a sketch of the projection of four representative trajectories (light curves) onto a Poincaré section (i.e., a plane intersecting transversally the limit cycle) in a neighborhood of the limit cycle. The figure's mutually perpendicular directions (bold lines) of stability (inward pointing arrows) and instability (outward pointing arrows) can form, in general, an arbitrary angle with each other. The shape of such a limit cycle need not be elliptic, as shown in the figure, except near the Hopf bifurcation point where it arises. Reprinted from Ghil and Yiou [1996] with permission of Springer-V erlag.

extended time intervals [Legras and Ghil, 1985]. Likewise, a ghost limit cycle is a closed orbit that has become slightly unstable but is visited, again and again, by the system's trajectories [Kimoto and Ghil, 1993].

[28] Consider the periodic solution shown in Figure la as embedded in Euclidean three-dimensional phase space. It is neutrally stable in the direction tangent to itself, while in the plane perpendicular to this tangent it is asymptotically stable in one direction and unstable in the other, as shown in the Poincaré section of F igure $1 b$. In a multidimensional phase space it is plausible that the directions of stability are numerous or even infinite in number. The directions of instability, however, would still be few in number, for parameter values not too far from those at which the $\mathrm{H}$ opf bifurcation that gave rise to the limit cycle in the first place occurs. Hence solutions of the full system would easily be attracted to this barely unstable limit cycle, follow it closely for one or a few turns, be ejected from its neighborhood, only to return later, again and again.

[29] The analogous picture for a ghost fixed point was illustrated in detail for an atmospheric model with 25 D OF by Legras and Ghil [1985; see also Ghil and Childress, 1987, Figures 6.12 and 6.18]. Thus the "ghosts" of fixed points and limit cycles leave their imprint on the system's observed spatiotemporal behavior.

[30] The episodes during which the system trajectory circles near a ghost limit cycle result in nearly periodic segments of the time series and hence contribute to a spectral peak with that period. This concept was illustrated using 40 years of an atmospheric multivariate time series by Kimoto and Ghil [1993] for the so-called intraseasonal oscillations of the Northern Hemisphere [see also Ghil and Mo, 1991a]. We shall show in the subsequent sections of the present review, in particular section 2.2, how this concept can be generalized to associate multiple spectral peaks with a robust skeleton of the attractor, as proposed by Vautard and Ghil [1989].

\section{ENHANCING THE SIGNAL-TO-NOISE (S/N) RATIO}

\subsection{Motivation for Singular Spectrum Analysis (SSA)}

[31] SSA is designed to extract information from short and noisy time series and thus provide insight into the unknown or only partially known dynamics of the underlying system that generated the series [Broomhead and King, 1986a; Fraedrich, 1986; Vautard and Ghil, 1989]. We outline here the method for univariate time series and generalize for multivariate ones in section 4.2.

[32] The analogies between SSA and spatial E OF s are summarized in A ppendix $A$, along with the basis of both in the Karhunen-Loève theory of random fields and of stationary random processes. Multichannel SSA (see section 4.2) is numerically analogous to the extended E OF (EE OF) algorithm of Weare and Nasstrom [1982]. The two different names arise from the origin of the former in the dynamical systems analysis of univariate time series, while the latter had its origins in the principal component analysis of meteorological fields. The two approaches lead to different methods for the choice of key parameters, such as the fixed or variable window width, and hence to differences in the way of interpreting results.

[33] The starting point of SSA is to embed a time series $\{X(t): t=1, \ldots, N\}$ in a vector space of dimension $M$, i.e., to represent it as a trajectory in the phase space of the hypothetical system that generated $\{X(t)\}$. In concrete terms this is equivalent to representing the behavior of the system by a succession of overlapping "views" of the series through a sliding $M$-point window.

[34] Let us assume, for the moment, that $X(t)$ is an observable function $\hat{X}(t)$ of a noise-free system's dependent variables $X_{i}(t)$, as defined in (4), and that the function $\phi$ that maps the $p$ variables $\left\{X_{i}(t): i=1, \ldots\right.$, $p\}$ into the single variable $X(t)$ has certain properties that make it generic in the dynamical systems sense of Smale [1967]. A ssume, moreover, that $M>2 d+1$, where $d$ is the dimension of the underlying attractor on which the system evolves, and that $d$ is known and finite. If so, then the representation of the system in the "delay coordinates" described in (5) below will share key topological properties with a representation in any coordinate system. This is a consequence of Whitney's [1936] embedding lemma and indicates the potential value of SSA in the qualitative analysis of the dynamics of nonlinear systems [Broomhead and King, 1986a, 1986b; Sauer et al., 1991]. The quantitative interpretation of SSA results in terms of attractor dimensions is fraught with difficulties, however, as pointed out by a number of authors [Broomhead et al., 1987; Vautard and Ghil, 1989; Paluš and Dvorák, 1992]. 
[35] We therefore use SSA here mainly (1) for dataadaptive signal-to-noise ( $\mathrm{S} / \mathrm{N})$ enhancement and associated data compression and (2) to find the attractor's skeleton, given by its least unstable limit cycles (see again Figure 1 above). The embedding procedure applied to do so constructs a sequence $\{\tilde{\mathbf{X}}(t)\}$ of $M$ dimensional vectors from the original time series $X$, by using lagged copies of the scalar data $\{X(t): 1 \leq t \leq$ $N\}$,

$$
\tilde{\mathbf{X}}(t)=(X(t), X(t+1), \ldots, X(t+M-1)) ;
$$

the vectors $\tilde{\mathbf{X}}(t)$ are indexed by $t=1, \ldots, N^{\prime}$, where $N^{\prime}$ $=N-M+1$.

[36] SSA allows one to unravel the information embedded in the delay-coordinate phase space by decomposing the sequence of augmented vectors thus obtained into elementary patterns of behavior. It does so by providing data-adaptive filters that help separate the time series into components that are statistically independent, at zero lag, in the augmented vector space of interest. These components can be classified essentially into trends, oscillatory patterns, and noise. As we shall see, it is an important feature of SSA that the trends need not be linear and that the oscillations can be amplitude and phase modulated.

[37] SSA has been applied extensively to the study of climate variability, as well as to other areas in the physical and life sciences. The climatic applications include the analysis of paleoclimatic time series [Vautard and Ghil, 1989; Yiou et al., 1994, 1995], interdecadal climate variability [Ghil and Vautard, 1991; Allen and Smith, 1994; Plaut et al., 1995; Robertson and Mechoso, 1998], as well as interannual [Rasmusson et al., 1990; Keppenne and Ghil, 1992] and intraseasonal [Ghil and Mo, 1991a, 1991b] oscillations. SSA algorithms and their properties have been investigated further by Penland et al. [1991], Allen [1992], Vautard et al. [1992], and Yiou et al. [2000]. The SSA Toolkit first documented by Dettinger et al. [1995a] was built, largely but not exclusively, around this technique.

\subsection{Decomposition and Reconstruction}

[38] In this section we illustrate the fundamental SSA formulae with the classical example of a climatic time series, the Southern Oscillation Index (SOI). SOI is a climatic index connected with the recurring EI Niño conditions in the tropical Pacific. It is defined usually as the difference between the monthly means of the sea level pressures at Tahiti and at Darwin (A ustralia). We use this definition and the monthly data given in the archive http://tao.atmos.washington.edu/pacs/additional analyses/soi.html.

[39] The SOI data in this archive are based on the time series at each of the two stations being deseasonalized and normalized [Ropelewski and Jones, 1987]. The seasonal cycle is removed by subtracting the average of the values for each calendar month over a reference

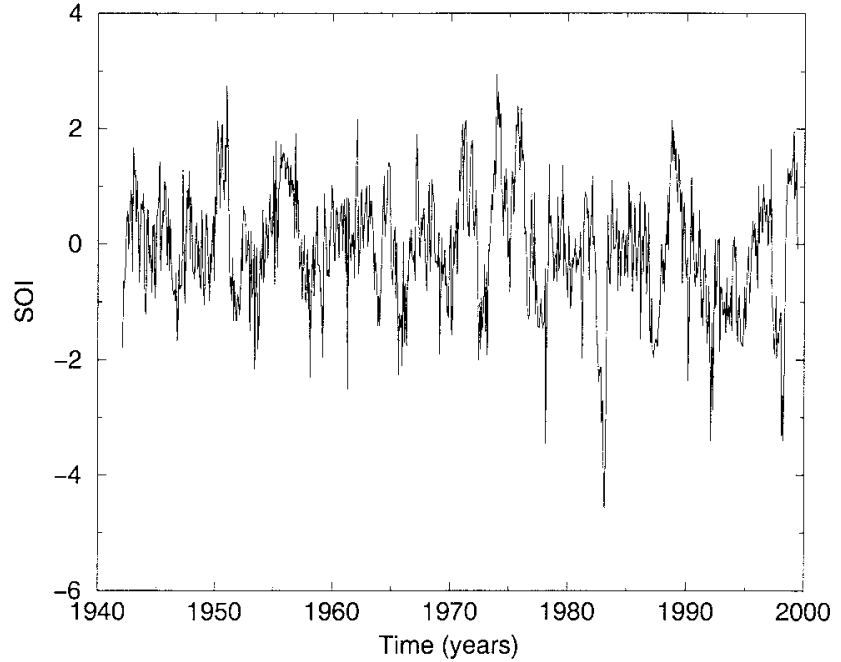

Figure 2. V ariations of the Southern O scillation Index (SOI) between J anuary 1942 and J une 1999. Time on the abscissa is in calendar years, and SOI on the ordinate is normalized by its standard deviation.

interval, in this case 1951-1980. The residues from this operation, called monthly anomalies in the climatological literature, are then normalized with respect to the standard deviation computed over the same interval.

[40] The archived SOI data for 1866-1997 are from the Climate Research $U$ nit of the U niversity of $E$ ast Anglia, and those for 1998-1999 are from the Climate Prediction Center of the U.S. N ational Centers for $\mathrm{En}$ vironmental Prediction (NCEP). They are obtained by taking the difference between the anomalies at Tahiti and those at $\mathrm{D}$ arwin and dividing by the standard deviation of this difference over the same 30-year reference interval. The time interval we consider here goes from J anuary 1942 to J une 1999, during which no observations are missing at either station; this yields $N=690$ raw data points. Note that this raw SOI time series is centered and normalized over the reference interval 19511980, but not over the entire interval of interest. We show in Figure 2 the SOI obtained from this raw data set. It actually has mean -0.0761 and standard deviation equal to 1.0677. A ll subsequent figures, however, use a version of the series that has been correctly centered over the interval J anuary 1942 to J une 1999.

[41] SSA is based on calculating the principal directions of extension of the sequence of augmented vectors $\left\{\tilde{\mathbf{X}}(t): t=1, \ldots, N^{\prime}\right\}$ in phase space. The $M \times M$ covariance matrix $\mathbf{C}_{X}$ can be estimated directly from the data as a Toeplitz matrix with constant diagonals; that is, its entries $c_{i j}$ depend only on the lag $|i-j|$ [cf. Vautard and Ghil, 1989]:

$$
c_{i j}=\frac{1}{N-|i-j|} \sum_{t=1}^{N-|i-j|} X(t) X(t+|i-j|) .
$$

The eigenelements $\left\{\left(\lambda_{k}, \rho_{k}\right): k=1, \ldots, M\right\}$ of $\mathbf{C}_{X}$ are then obtained by solving 


$$
\mathbf{C}_{X} \rho_{k}=\lambda_{k} \rho_{k}
$$

The eigenvalue $\lambda_{k}$ equals the partial variance in the direction $\rho_{k}$, and the sum of the $\lambda_{k}$, i.e., the trace of $\mathbf{C}_{X}$, gives the total variance of the original time series $X(t)$.

[42] A $n$ equivalent formulation of (7), which will prove useful further on, is given by forming the $M \times M$ matrix $\mathbf{E}_{X}$ that has the eigenvectors $\rho_{k}$ as its columns and the diagonal matrix $\Lambda_{X}$ whose elements are the eigenvalues $\lambda_{k}$, in decreasing order:

$$
\mathbf{E}_{X}^{\mathrm{t}} \mathbf{C}_{X} \mathbf{E}_{X}=\Lambda_{X} ;
$$

here $\mathbf{E}_{X}^{\mathrm{t}}$ is the transpose of $\mathbf{E}_{X}$. E ach eigenvalue $\lambda_{k}$ gives the degree of extension, and hence the variance, of the time series in the direction of the orthogonal eigenvector $\rho_{k}$.

[43] A slightly different approach to computing the eigenelements of $\mathbf{C}_{X}$ was originally proposed by Broomhead and King [1986a]. They constructed the $N^{\prime} \times M$ trajectory matrix $\mathbf{D}$ that has the $N^{\prime}$ augmented vectors $\tilde{\mathbf{X}}(t)$ as its rows and used singular-value decomposition (SV D) [see, for instance, Golub and Van Loan, 1996] of

$$
\mathbf{C}_{X}=\frac{1}{N^{\prime}} \mathbf{D}^{\mathrm{t}} \mathbf{D}
$$

to obtain the square roots of $\lambda_{k}$. The latter are called the singular values of $\mathbf{D}$ and have given SSA its name.

[44] Allen and Smith [1996] and Ghil and Taricco [1997] have discussed the similarities and differences between the approaches of Broomhead and King [1986a] and Vautard and Ghil [1989] in computing the eigenelements associated in SSA with a given time series $X(t)$. Both the Toeplitz estimate (6) and the SV D estimate (9) lead to a symmetric covariance matrix $\mathbf{C}_{X}$. In addition, the eigenvectors $\rho_{k}$ of a Toeplitz matrix are necessarily odd and even, like the sines and cosines of classical Fourier analysis. The Toeplitz approach has the advantage of better noise reduction when applied to short time series, as compared with the SVD approach. This advantage comes at the price of a slightly larger bias when the time series is strongly nonstationary over the interval of observation $1 \leq t \leq N$ [Allen, 1992]. Such biasversus-variance trade-offs are common in estimation problems.

[45] To obtain $\mathrm{S} / \mathrm{N}$ separation, one plots the eigenvalue spectrum illustrated in Figure 3 . In this plot an initial plateau that contains most of the signal is separated by a steep slope from the noise; the latter is characterized by much lower values that form a flat floor or a mild slope [Kumaresan and Tufts, 1980; Pike et al., 1984; Vautard and Ghil, 1989].

[46] As the $M \times M$ matrix $\mathbf{C}_{X}$ is symmetric, standard algorithms [Press et al., 1988] will perform its spectral decomposition efficiently, as long as $M$ is not too large. The choice of $M$ is based on a trade-off between two considerations: quantity of information extracted versus the degree of statistical confidence in that information.

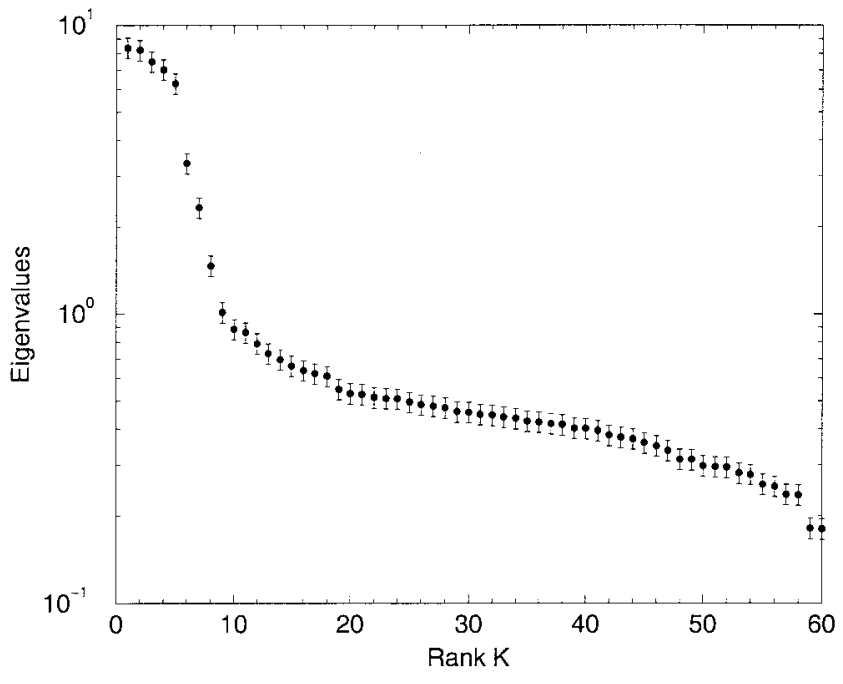

Figure 3. Singular spectrum of the SOI time series. The eigenvalues are plotted, as usual, in decreasing order. The embedding dimension for Figures $3-7$ is $M=60$, which represents a time window of 5 years. The error bars are based on the default option of the singular spectrum analysis (SSA) multitaper method (MTM) Toolkit (http://www.atmos.ucla.edu/tcd/ssa/). This option uses the ad hoc estimate of variance $\sigma_{k}$ of an eigenvalue $\lambda_{k}$ given by $\sigma_{k}=(2 / \hat{N}) \lambda_{k}$. The key element of this estimate is the number $\hat{N}$ of independent degrees of freedom (DOF) in the time series. Vautard and Ghil [1989], who first proposed this estimate in SSA, used $\hat{N}=N$, which is very optimistic, while Ghil and Mo [1991a] used $\hat{N}=$ $N / M$, which is too conservative. The Toolkit's current V ersion 4.0, following Unal and Ghil [1995], uses $\hat{N}=N / \kappa \tau$, where $\tau$ is the time series' estimated decorrelation time (see equations (15) and (16)) and $\kappa$ is an empirical constant, $\kappa=1.5$.

The former requires as wide a window as possible, i.e., a large $M$, while the latter requires as many repetitions of the features of interest as possible, i.e., as large a ratio $N / M$ as possible. The choice of $M=60$ in Figure 3 allows us to capture periodicities as long as 5 years, since $\Delta t=1$ month, and thus the dimensional window width is $M \Delta t=5$ years; on the other hand, $N / M \cong 11$ is fairly safe, and the diagonalization of $\mathbf{C}_{X}$ for this moderate value of $M$ does not introduce large numerical errors either.

[47] In Figure 3, there is a clear grouping of the first five eigenvalues, followed by a very steep slope of three additional eigenvalues. The latter are well separated from the first five, as well as from the remaining 52 eigenvalues, which form the mildly sloping and flattening out "tail" of the SSA spectrum.

[48] The S/N separation obtained by merely inspecting the slope break in a "scree diagram" of eigenvalues $\lambda_{k}$ or singular values $\lambda_{k}^{1 / 2}$ versus $k$ works well when the intrinsic noise that perturbs the underlying deterministic system and the extrinsic noise that affects the observations are both white, i.e., uncorrelated from one time step to the next (see definition of $\xi(t)$ in equation (1)). This rudimentary separation works less well when either 


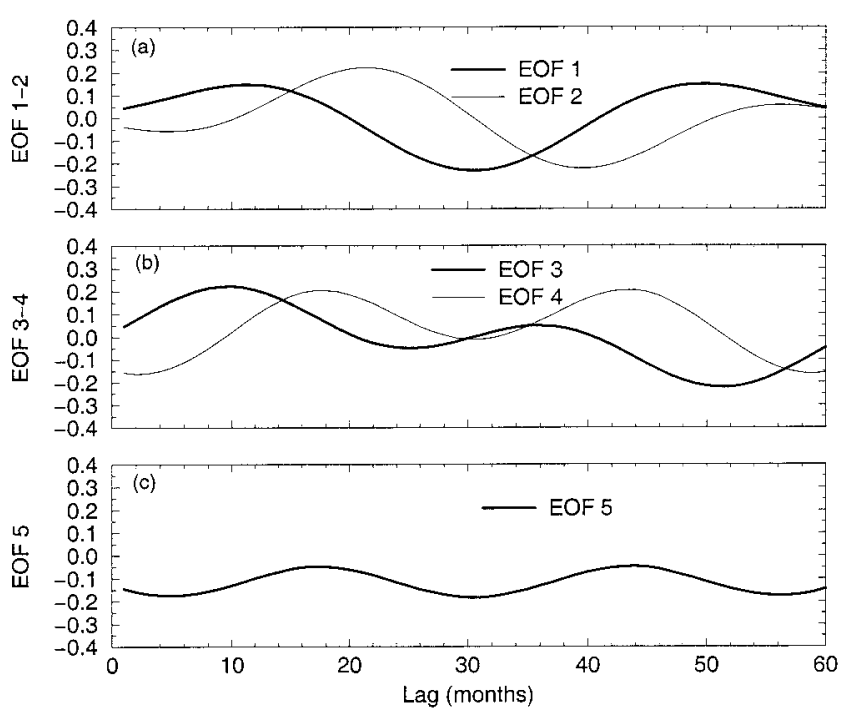

Figure 4. First five empirical orthogonal functions (EOFs) of the SOI time series. The leading four EOFs are grouped into two pairs, $(1,2)$ and $(3,4)$, in Figures $4 a$ and $4 b$.

noise is red, i.e., when it is given by an A R (1) process (see section 3.3) or is otherwise correlated between time steps [Vautard and Ghil, 1989]. The difficulties that arise with correlated noise led Allen [1992] and Allen and Smith [1994] to develop M onte Carlo SSA (see section 2.3).

[49] When the noise properties can be estimated reliably from the available data, the application of a socalled "prewhitening operator" can significantly enhance the signal separation capabilities of SSA [Allen and Smith, 1997]. The idea is to preprocess the time series itself or, equivalently but often more efficiently, the lag covariance matrix $\mathbf{C}_{X}$, such that the noise becomes uncorrelated in this new representation. SSA is then performed on the transformed data or covariance matrix and the results are transformed back to the original representation for inspection.

[50] By analogy with the meteorological literature, the eigenvectors $\rho_{k}$ of the lag covariance matrix $\mathbf{C}_{X}$ have been called empirical orthogonal functions (EOFs) [see Preisendorfer, 1988, and references therein] by Fraedrich [1986] and by Vautard and Ghil [1989]. The EOF s corresponding to the first five eigenvalues are shown in Figure 4. N ote that the two EOF s in each one of the two leading pairs, i.e., EOFs 1 and 2 (Figure 4a) as well as EOFs 3 and 4 (Figure $4 \mathrm{~b}$ ), are in quadrature and that each pair of EOFs corresponds in Figure 3 to a pair of eigenvalues that are approximately equal and whose error bars overlap. Vautard and Ghil [1989] argued that subject to certain statistical significance tests discussed further below, such pairs correspond to the nonlinear counterpart of a sine-cosine pair in the standard Fourier analysis of linear problems.

[51] In the terminology of section 1 here, such a pair gives a handy representation of a ghost limit cycle. The advantage over sines and cosines is that the EOFS

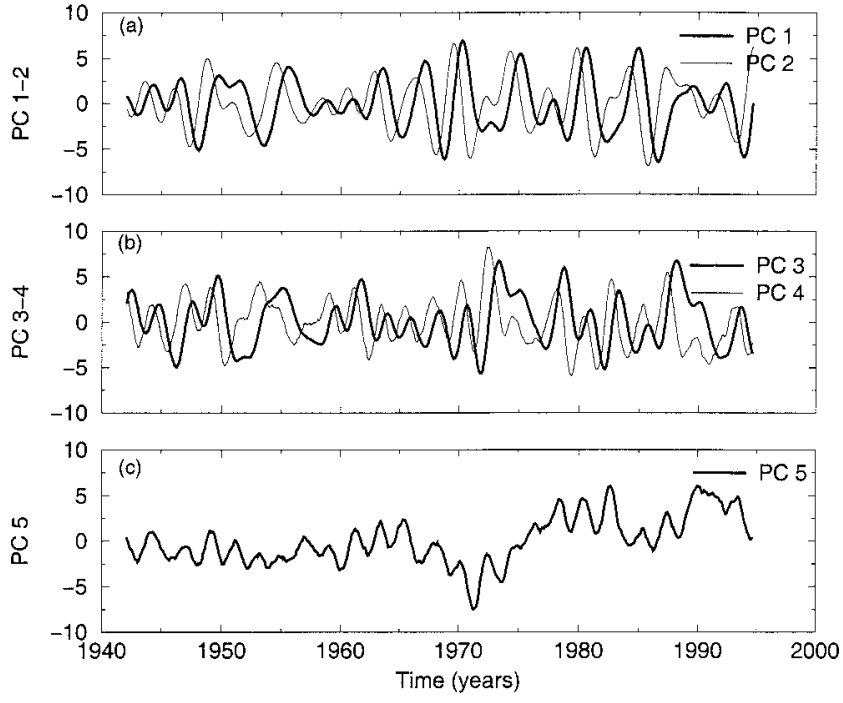

Figure 5. First five principal components (PCS) of the SOI time series. Note phase quadrature in Figures $5 \mathrm{a}$ and $5 \mathrm{~b}$. The maximal cross correlation in Figure $5 \mathrm{a}$ is reached when $\mathrm{PC}-2$ leads PC -1 by 10 months and equals 0.89 . In Figure $5 b$ the maximum cross correlation equals 0.71 and is obtained when PC -4 leads PC -3 by 9 months.

obtained from SSA are not necessarily harmonic functions and, being data adaptive, can capture highly anharmonic oscillation shapes. Indeed, relaxation oscillations [Van der Pol, 1940] and other types of nonlinear oscillations [Stoker, 1950], albeit purely periodic, are usually not sinusoidal; that is, they are anharmonic. Such nonlinear oscillations often require therefore the use of many harmonics or subharmonics of the fundamental period when carrying out classical Fourier analysis, while a single pair of SSA eigenmodes might suffice. Capturing the shape of an anharmonic oscillation, such as a seesaw or boxcar, albeit slightly rounded or smoothed, is easiest when the SSA window is exactly equal to the single period being analyzed.

[52] Projecting the time series onto each E OF yields the corresponding principal components (PCS) $\mathbf{A}_{k}$ :

$$
A_{k}(t)=\sum_{j=1}^{M} X(t+j-1) \rho_{k}(j) .
$$

Figure 5 shows the variations of the five leading PCs. A gain, the two PCs in each of the pairs $(1,2)$ and $(3,4)$ are in quadrature, two by two (see Figures $5 a$ and $5 b$ ). They strongly suggest periodic variability at two different periods, of about 4 and 2 years, respectively. Substantial amplitude modulation at both periodicities is present, too.

[53] The fifth PC, shown in Figure $5 c$, contains both a long-term, highly nonlinear trend and an oscillatory component. We shall discuss the trend of the SOI series in connection with Figures $6 \mathrm{~b}$ and 16a further below.

[54] We can reconstruct that part of a time series that 

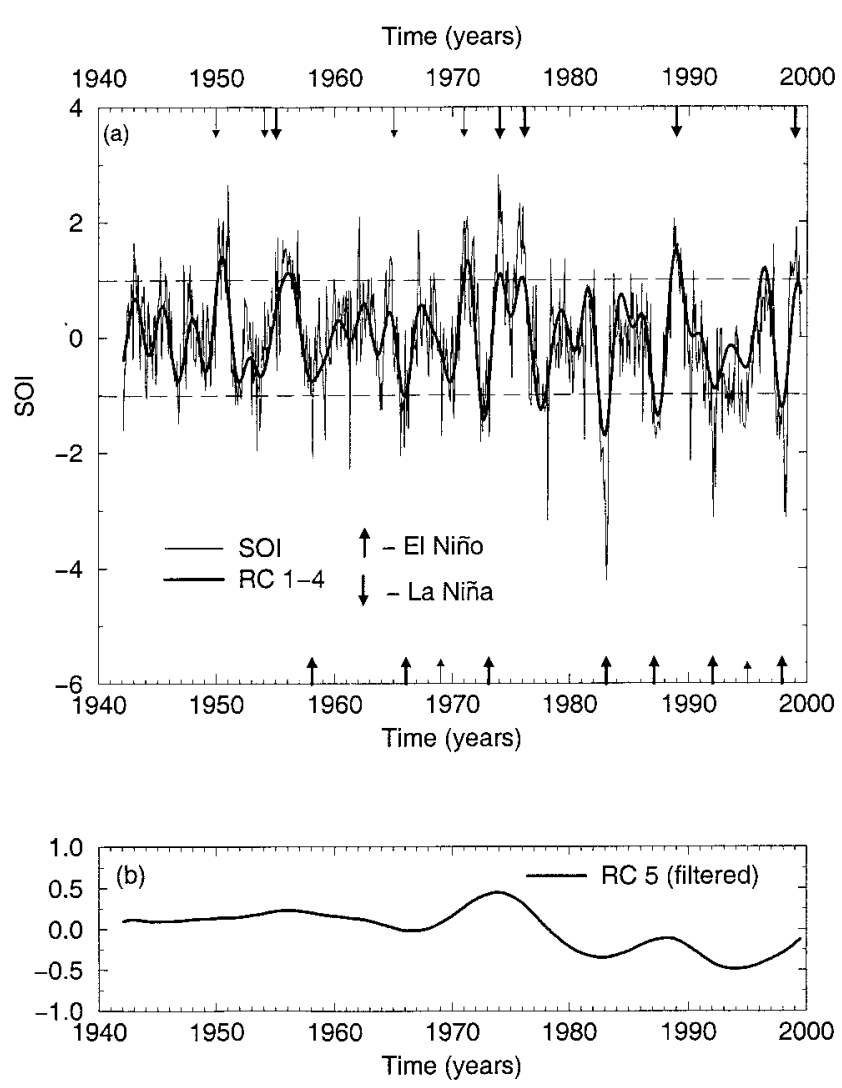

Figure 6. Partial reconstructions of the SOI time series. Figure $6 a$ is a reconstruction based on EOFs 1-4 (bold curve). The raw SOI series is shown as the light curve. EI N iños (warm events) are shown as upward pointing arrows on the lower abscissa, while La Niñas (cold events) are shown as downward pointing arrows on the upper abscissa; the arrows are aligned with the January closest to the event maximum. Figure $6 b$ shows the trend of reconstructed component (RC) number 5 (R C-5); see text for details.

is associated with a single EOF or several by combining the associated PCs:

$$
R_{\mathscr{K}}(t)=\frac{1}{M_{t}} \sum_{k \in \mathscr{K}} \sum_{j=L_{t}}^{U_{t}} A_{k}(t-j+1) \rho_{k}(j) ;
$$

here $\mathscr{K}$ is the set of EOF s on which the reconstruction is based. The values of the normalization factor $M_{t}$, as well as of the lower and upper bound of summation $L_{t}$ and $U_{t}$, differ between the central part of the time series and its end points [Ghil and Vautard, 1991; Vautard et al., 1992]:

$$
\begin{aligned}
& \left(M_{t}, L_{t}, U_{t}\right)= \\
& \left\{\begin{array}{cc}
\left(\frac{1}{t}, 1, t\right), & 1 \leq t \leq M-1, \\
\left(\frac{1}{M}, 1, M\right), & M \leq t \leq N^{\prime}, \\
\left(\frac{1}{N-t+1}, t-N+M, M\right), & N^{\prime}+1 \leq t \leq N .
\end{array}\right.
\end{aligned}
$$

[55] The reconstructed components (RCS) have the property of capturing the phase of the time series in a well-defined least squares sense, so that $X(t)$ and $R_{\mathscr{K}}(t)$ can be superimposed on the same timescale, $1 \leq t \leq N$. This is an advantage of the RCs over the PCs, which have length $N-M$ and do not contain direct phase information within the window width $M$.

[56] No information is lost in the reconstruction process, since the sum of all individual R Cs gives back the original time series. Partial reconstruction is illustrated in Figure 6 by summing the variability of PCS 1-4, associated with the two leading pairs of eigenelements; it is common to refer to such a reconstruction (equation (11)), with $\mathscr{K}=\{1,2,3,4\}$, as R Cs $1-4$. The portion of the SOI variability thus reconstructed contains $43 \%$ of the total variance. It captures the quasi-oscillatory behavior isolated by these two leading pairs, with its two distinct near-periodicities.

[57] It is clear that the partial SOI reconstruction (bold curve in Figure 6a) is smooth and represents the essential part of the interannual variability in the monthly SOI data (thin curve). E ach of the two pairs of R Cs, 1-2 and 3-4, can be thought of as retracing a ghost limit cycle in the phase space of the tropical climate system. These two limit cycles can then be said to form the robust skeleton of the attractor. It is unlikely that a time series of a few hundred points, like the SO I or other typical climatic time series, will suffice to capture the attractor's fine structure [Vautard and Ghil, 1989].

[58] This robust skeleton, however, provides sufficient information for most practical purposes. In particular, warm events (EI Niños) and cold ones (La Niñas) over the eastern tropical Pacific are captured quite well, during the 57.5 years of record, as minima and maxima of the partially reconstructed $\mathrm{SO}$ I. W e check this statement by comparing the bold curve in Figure $6 a$ with the vertical arrows along the figure's upper and lower abscissae.

[59] These arrows correspond to strong (bold arrows) or moderate (light arrows) EI Niño-Southern O scillation (ENSO) events. The events are defined subjectively, calendar quarter by calendar quarter, from reanalyzed surface temperature data produced at NCEP and the U.K. M et. Office for the years 1950-1999 (see http:// www.cpc.noaa.gov/products/analysis_monitoring/ensostuff/ ensoyears.html).

[60] Large positive peaks in the partial SOI reconstruction, i.e., those that exceed one standard deviation, match the strong La Niñas quite well (downward pointing arrows on the upper abscissa). The only exceptions are the 1950 and 1971 cold events, which were of moderate strength, and the weak 1996 La Niña.

[61] The same good match obtains between the large negative peaks in the figure's bold curve, i.e., those that exceed one standard deviation, and the strong $\mathrm{EI} \mathrm{N}$ iños (upward pointing arrows on the lower abscissa). The only notable exception is the large peak in 1977-1978, which was classified subjectively as a weak warm event. 
The 1957-1958 and 1991-1992 events appear as moderate-size minima in the partial SOI reconstruction. They are included in the NCE P list as strong EI Niños for one (J anuary-M arch 1958) or two (J anuary-J une 1992) seasons, but neither was strong during the second half of the calendar year. Thus the only discrepancies among the oscillatory part of the SOI, based on R C S 1-4, and the subjective NCEP classification are in the intensities (moderate versus strong, or vice versa) of a few events.

[62] Earlier SSA results do support the present emphasis on the doubly periodic character of ENSO phenomena. They include the analyses of Rasmusson et al. [1990] for sea surface temperatures and near-surface zonal winds in the tropical Indo-Pacific belt, those of Keppenne and Ghil [1992] for a slightly different treatment of the SOI, as well as those of Jiang et al. [1995a] for sea surface temperatures and of Unal and Ghil [1995] for sea level heights in the tropical Pacific. In all these data sets and SSA analyses, a quasi-biennial and a lowerfrequency, quasi-quadrennial oscillatory pair were reliably identified among the leading SSA eigenelements.

[63] Shown in Figure $6 \mathrm{~b}$ is also a filtered version of R C -5, which captures well the small but significant longterm trend of the SOI time series in Figure 2. To eliminate the oscillatory component apparent in PC-5 (Figure 5c), we applied SSA with the same 60-month window to the full RC-5. The two leading eigenmodes correspond to a pure trend, shown in Figure $6 \mathrm{~b}$, while the second eigenpair corresponds to a quasi-biennial oscillation (not shown). The SOI trend in Figure $6 \mathrm{~b}$ agrees, up to a point, with the one captured by the multitaper reconstruction in section 3.4 .2 (see Figure 16 a there). Given the recent interest in the interdecadal variability of ENSO, we postpone further discussion of this result for the moment when its multitaper version is also in hand.

[64] R eliable S/N separation and identification of oscillatory pairs is not always as easy as in the case of interannual climate variability in the tropical Pacific. G lobal surface-air temperatures, for instance, present a considerably more difficult challenge for identifying interannual and interdecadal oscillations. Elsner and Tsonis's [1991] excessive reliance on eigenvalue rank order as a criterion of significance in SSA has led to considerable confusion in this case [see Allen et al., 1992a, 1992b].

[65] R eliable identification of the true signal conveyed by a short, noisy time series and of the oscillatory components within this signal requires effective criteria for statistical significance, which are treated in the next section. Subject to these caveats, a clean signal, obtained by partial reconstruction over the correct set of indices $\mathscr{K}$, provides very useful information on the underlying system, which is often poorly or incompletely known.

[66] Such a signal can then be analyzed further, both visually and by using other spectral analysis tools that are described in section 3. The maximum entropy method (MEM), which we describe in section 3.3, works partic- ularly well on signals so enhanced by SSA [Penland et al., 1991].

\subsection{Monte Carlo SSA}

[67] In the process of developing a methodology for applying SSA to climatic time series, a number of heuristic [Vautard and Ghil, 1989; Ghil and Mo, 1991a; Unal and Ghil, 1995] or M onte Carlo [Ghil and Vautard, 1991; Vautard et al., 1992] methods have been devised for $S / N$ separation or the reliable identification of oscillatory pairs of eigenelements. They are all essentially attempts to discriminate between the significant signal as a whole, or individual pairs, and white noise, which has a flat spectrum. A more stringent "null hypothesis" [Allen, 1992] is that of red noise, since most climatic and other geophysical time series tend to have larger power at lower frequencies [Hasselmann, 1976; Mitchell, 1976; Ghil and Childress, 1987].

[68] For definiteness, we shall use here the term red noise exclusively in its narrow sense, of an A R (1) process given by (1) with $M=1$ and $0<a_{1}<1$, as required by weak or wide-sense stationarity (see Appendix A for an exact definition). Other stochastic processes that have a continuous spectral density $S(f)$ which decreases monotonically with frequency $f$ will be called "warm colored."

[69] The power spectrum $S(f)$ of the AR (1) process is given by [e.g., Chatfield, 1984]

$$
S(f)=S_{0} \frac{1-r^{2}}{1-2 r \cos \left(2 \pi f / f_{N}\right)+r^{2}} .
$$

Here $0<S_{0}<\infty$ is the average value of the power spectrum, related to the white-noise variance $\sigma^{2}$ in (1) by

$$
S_{0}=\frac{\sigma^{2}}{1-r^{2}},
$$

while $r$ is the lag-one autocorrelation, $r=a_{1}$, and the $N$ yquist frequency $f_{N}=1 /(2 \Delta t)$ is the highest frequency that can be resolved for the sampling rate $\Delta t$. N ote that in (1) and (5) we have used $\Delta t=1$ for simplicity and without loss of generality, since $\Delta t$ can always be redefined as the time unit. It is useful at this point to recall, for clarity's sake, that it is not necessary to do so. The characteristic decay timescale $\tau$ of the AR (1) noise can be estimated by

$$
\tau=-\frac{\Delta t}{\log r} .
$$

[70] In general, straightforward tests can be devised to compare a given time series with an idealized noise process: The continuous spectrum of such a process is known to have a particular shape, and if a particular feature of the data spectrum lies well above this theoretical noise spectrum, it is often considered to be statistically "significant." A single realization of a noise process can, however, have a spectrum that differs greatly from the theoretical one, even when the number of data points is large. It is only the (suitably weighted) 
average of such sample spectra over many realizations that will tend to the theoretical spectrum of the ideal noise process. Indeed, the Fourier transform of a single realization of a red-noise process can yield arbitrarily high peaks at arbitrarily low frequencies; such peaks could be attributed, quite erroneously, to periodic components.

[71] M ore stringent tests have to be used therefore to establish whether a time series can be distinguished from red noise or not. Allen [1992] devised such a test that compares the statistics of simulated red-noise time series with those of a given climatic time series. The principle of this test is implicit in some of Broomhead and King's [1986a] ideas. The application of SSA in combination with this particular M onte Carlo test against red noise has become known as "M onte Carlo SSA" (M C-SSA) [see Allen and Smith, 1994, 1996].

[72] M C-SSA can be used, more generally, to establish whether a given time series can be distinguished from other well-defined processes. We only present here, for the sake of brevity and clarity, the original test against an A R (1) process. Allen [1992] proposes, in fact, to estimate the mean $X_{0}$ of the process at the same time as the other parameters. We therefore rewrite (1) here for the particular case at hand as

$$
X(t)=a_{1}\left[X(t-1)-X_{0}\right]+\sigma \xi(t)+X_{0} ;
$$

here, as in (1), $\xi(t)$ is a $\mathrm{G}$ aussian-distributed white-noise process with zero mean and unit variance.

[73] When testing against the process (16), the first step in MC-SSA is to estimate the mean $X_{0}$ and the coefficients $a_{1}$ and $\sigma$ from the time series $X(t)$ by using a maximum likelihood criterion. Allen and Smith [1996] provide low-bias estimators that are asymptotically unbiased in the limit of large $N$ and close to unbiased for series whose length $N$ is at least an order of magnitude longer than the decorrelation time $\tau=-1 / \log r$. Cochrane and Orcutt [1949] have shown that when $\tau$ is not very small relative to $N$, the use of a crude estimate for the mean $X_{0}$, i.e., "centering" the time series first, can lead to severe biases in the subsequent estimation of $a_{1}$. This is not the case for the SOI time series used here, as $\tau \ll N$ for it. Hence we have used an SOI time series, based on the data in Figure 2, that has been centered.

[74] On the basis of estimated values $\hat{X}_{0}, \hat{a}_{1}$, and $\hat{\sigma}$ of these parameters, an ensemble of simulated red-noise data is generated and, for each realization, a covariance matrix $\mathbf{C}_{R}$ is computed. In the nonlinear dynamics literature, such simulated realizations of a noise process are often called surrogate data [Drazin and King, 1992; Ott et al., 1994].

[75] The covariance matrices of the surrogate data are then projected onto the eigenvector basis $\mathbf{E}_{X}$ of the original data by using (8) for their SVD,

$$
\Lambda_{R}=\mathbf{E}_{X}^{t} \mathbf{C}_{R} \mathbf{E}_{X} .
$$

[76] Since (17) is not the SVD of the particular realization $\mathbf{C}_{R}$, the matrix $\Lambda_{R}$ is not necessarily diagonal, as

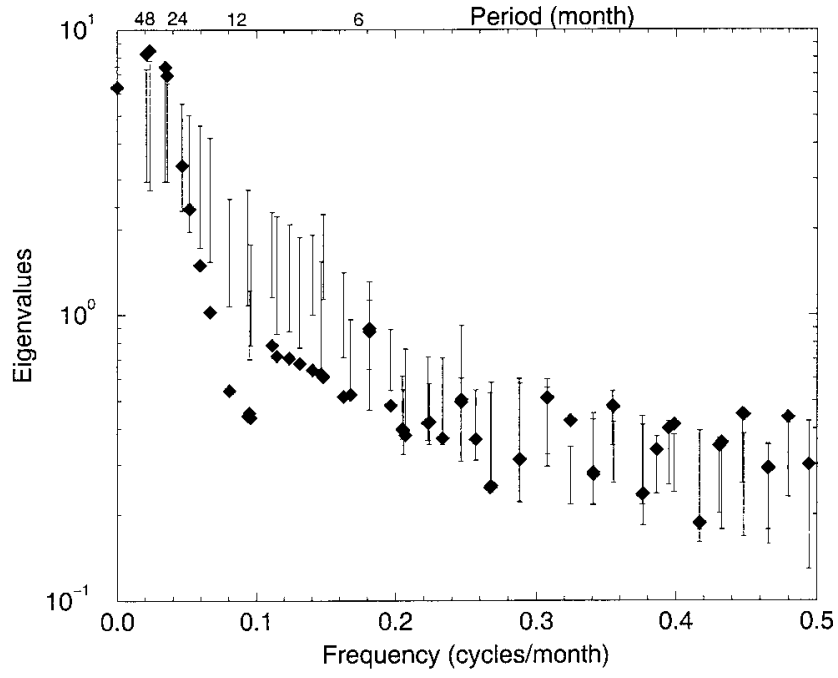

Figure 7. M onte Carlo singular spectrum of the SOI time series. The diamonds indicate projections of the noise eigenvectors onto the data correlation matrix (see equation (17)); the lower and upper ticks on the error bars indicate the 5th and 95th noise percentiles. For each EOF, a characteristic frequency was estimated by maximizing its correlation with a sinusoid; therefore the frequency at which each diamond is plotted is just an average estimate. The frequencies in this figure and subsequent ones are given in cycles per month.

it is in (8). Instead, $\boldsymbol{\Lambda}_{R}$ measures the resemblance of a given surrogate set with the original data set of interest. The degree of resemblance can be quantified by computing the statistics of the diagonal elements of $\Lambda_{R}$. The statistical distribution of these elements, determined from the ensemble of M onte Carlo simulations, gives confidence intervals outside which a time series can be considered to be significantly different from a random realization of the process (16). For instance, if an eigenvalue $\lambda_{k}$ lies outside a $90 \%$ noise percentile, then the red-noise null hypothesis for the associated EOF (and PC) can be rejected with this level of confidence. 0 therwise, that particular SSA component of the time series cannot be considered as significantly different from red noise. A dditional problems posed by the multiplicity of SSA eigenvalues and other finer points are also discussed by Allen [1992] and Allen and Smith [1996].

[77] As the next step in the analysis of our SOI time series, we apply an M C-SSA noise test to it. In order to enhance the readability of the diagrams for the SSA spectra in the presence of M C-SSA error bars, we associate a dominant frequency with each EOF detected by SSA, as suggested by Vautard et al. [1992], and plot in Figure 7 the eigenvalues (diamonds) versus frequency, following Allen and Smith [1996].

[78] Such a plot is often easier to interpret, with respect to the M C-SSA error bars, than plotting versus the eigenvalue's rank $k$ as in Figure 3. Care needs to be exercised, however, since the dominant-frequency estimate may be ambiguous or uncertain, due to the possible anharmonicity of the EOFs, especially for low fre- 
quencies. This is patently the case for the fifth mode in Figures 3-5. This mode appears at zero frequency in Figure 7, while we know very well that it has a quasibiennial oscillatory component besides its capturing the SOI's nonlinear trend.

[79] The error bars shown in Figure 7 for each EOF represent $90 \%$ of the range of variance found in the state-space direction defined by that EOF in an ensemble of 1000 red-noise realizations; that is, it denotes the interval between the 5th and 95th percentile. Hence eigenvalues lying outside this interval are relatively unlikely (at the $10 \%$ level) to be due merely to the rednoise process (equation (16)) against which they are being tested.

[80] The high values in Figure 7 exhibit a significant quasi-biennial oscillation and an oscillatory component with a period of about 50 months. The low values near 1 cycle $\mathrm{yr}^{-1}$ are due to the fact that the seasonal cycle has been removed prior to the analysis, and the consequent suppression of power near annual-cycle periods has not been taken into account in the noise parameter estimation. Allen and Smith [1996] recommend that as far as possible, seasonal cycles should not be removed prior to the analysis, but that their presence should be taken into account explicitly in the parameter estimation [see also Jiang et al., 1995a; Unal and Ghil, 1995]. This recommendation has to be weighted against two related considerations. First, the reliable identification of a given periodicity becomes harder as the number of periodicities to be estimated increases, and second, the seasonal cycle and its physical causes are fairly well known and understood.

[81] The M C-SSA algorithm described above can be adapted to eliminate known periodic components and test the residual against noise. This adaptation can provide better insight into the dynamics captured by the data. Indeed, known periodicities, like orbital forcing on the Quaternary timescale or seasonal forcing on the intraseasonal-to-interannual one, often generate much of the variance at the lower frequencies manifest in a time series and alter the rest of the spectrum. Allen [1992] and Allen and Smith [1996] describe this refinement of M C-SSA which consists of restricting the projections given by (17) to the EOFs that do not account for known periodic behavior.

[82] M onte Carlo simulation is a robust, flexible, and nonparametric approach to assessing the significance of individual eigenmodes in SSA. Since it can be computationally intensive, Allen and Smith [1996] suggest a much faster, al beit parametric alternative. In an appendix they also clarify the relationship between confidence intervals from MC-SSA and earlier heuristic approaches [e.g., Vautard and Ghil, 1989; Ghil and Mo, 1991a; Unal and Ghil, 1995].

[83] M C-SSA provides, furthermore, the means of evaluating the significance of frequency separation between apparently distinct spectral peaks, without appealing to the application of additional spectral methods.
The associated "bandwidth" is $1 / M$ and is discussed further in a multichannel context in section 4.2. Given the fact that the assignment of frequency to an eigenpair is not entirely unique (see discussion of the zero-frequency component in Figure 7), we still recommend the application of other spectral methods for the detailed study of spectral content, in addition to SSA and M C-SSA.

\subsection{Multiscale SSA and Wavelet Analysis}

[84] Wavelet analysis has become a basic tool for the study of intermittent, complex, and self-similar signals, because it works as a mathematical microscope that can focus on a specific part of the signal to extract local structures and singularities [Strang, 1989; Meyer, 1992, 1993; Daubechies, 1992]. In climate dynamics [Meyers et al., 1993; Weng and Lau, 1994; Torrence and Compo, 1998], and geophysics [Kumar and Foufoula-Georgiou, 1997], wavelets have been used mostly to follow changes in frequency of one or more periodic signals. While SSA follows amplitude and phase modulation of a signal easily (see section 2.2, as well as Plaut and Vautard [1994] and Moron et al. [1998]), a narrow band of frequencies that vary in time, from one line in the band to another (see section 3.1), is captured typically by a single pair of SSA eigenmodes.

[85] A wavelet transform requires the choice of an analyzing function or "mother wavelet" $\psi$ that has general admissibility properties [Meyer, 1992; Daubechies, 1992], as well as the more specific property of time and frequency localization; that is, $\psi$ and its Fourier transform $\mathscr{F} \psi$ must decay rapidly outside a given interval. Functions $\psi$ based on a Gaussian, $\psi(x)=\exp \left(-x^{2}\right)$, first proposed in this context by Gabor [1946], possess the localization property even though they do not satisfy the admissibility condition that their integral over the real line $\mathbb{R}$ vanish [Delprat et al., 1992].

[86] A $\psi$-wavelet transform $W_{\psi}$ in continuous time and frequency is simply a projection of a signal $X(t),-\infty$ $<t<\infty$, onto $b$-translated and $a$-dilated versions of $\psi$ :

$$
W_{\psi}(a, b)=\frac{1}{\sqrt{a}} \int_{-\infty}^{\infty} X(t) \psi\left(\frac{t-b}{a}\right) d t .
$$

If most of $\psi$ is concentrated in the interval $[-1,1]$, say (up to a rescaling), then (18) is clearly an analysis of $X$ in the interval $[b-a, b+a]$. U sing the successive derivatives $\psi^{(n)}$ of a given mother wavelet $\psi$ in (18) is equivalent (up to a normalization factor) to a $\psi$ analysis of the successive derivatives of the time series $X$; this is easy to see through an integration by parts.

[87] The original signal, or a filtered version of it, can be reconstructed from the family of wavelet transforms. H ence for scale values $a$ in an interval $I$, a reconstructed version $X_{I}$ of the signal $X(t)$ is

$$
X_{I}(t)=A_{\psi} \int_{a \in I} \int_{b=-\infty}^{\infty} W_{\psi}(a, b) \psi\left(\frac{t-b}{a}\right) \frac{d a d b}{a^{2}} ;
$$


TABLE 2. Analogy Between SSA and Wavelet Analysis ${ }^{a}$

\begin{tabular}{lcc}
\hline \multicolumn{1}{c}{ Method } & $S S A$ & Wavelet Transform \\
\hline A nalyzing function & E OF $\rho_{k}$ & mother wavelet $\psi$ \\
Basic facts & $\rho_{k}$ eigenvectors of $\mathbf{C}_{X}$ & $\psi$ chosen a priori \\
Decomposition & $\sum_{t^{\prime}=1}^{M} X\left(t+t^{\prime}\right) \rho_{k}\left(t^{\prime}\right)$ & $\int X(t) \psi((t-b) / a)$ \\
Scale & $W=\alpha M \Delta t$ & $d t$ \\
E poch & $t$ & $a$ \\
A verage and trend & $\rho_{1}$ & $b$ \\
Derivative & $\rho_{2}$ & $\psi^{(0)}$ \\
\hline
\end{tabular}

asee text for notation.

$A_{\psi}$ is a normalization factor which only depends on the mother wavelet $\psi$. This formulation is essentially a bandpass filter of $X$ through $I$; if $I$ is the positive real line, $I=$ $\mathbb{R}^{+}$, then $X_{I}(t)=X(t)$. N ote that the G aussian $\psi(x)=$ $\exp \left(-x^{2} / 2\right)$ itself cannot be used in the reconstruction formula (19), because it does not satisfy $\int_{-\infty}^{\infty} \psi(x) d x=$ 0 , although its first derivative does. The forward transform of (18), however, is well defined, and the G aussian can be used as an analyzing tool [Arneodo et al., 1993; Yiou et al., 2000].

[88] A large number of wavelet bases have been introduced to satisfy the conflicting requirements of completeness, localization in both time and frequency, and orthogonality or, for nonorthogonal bases, limited redundancy. To provide an optimal multiscale decomposition of a given signal, an automatic time-varying adjustment of the mother wavelet's shape may be desirable. This could replace the current practice of searching through extensive "libraries" of mother wavelets (e.g., http://www.mathsoft.com/wavelets.html). To provide such a data-adaptive variation in basis with time, Yiou et al. [2000] have introduced multiscale SSA.

[89] Systematic comparisons between SSA, the wavelet transform, and other spectral analysis methods have been carried out by Yiou et al. [1996] and in Table 1 of Ghil and Taricco [1997]. Further analogies between certain mathematical features of SSA and wavelet analysis were mentioned by Yiou [1994]. Table 2 here summarizes the most useful mathematical parallels between the two time series analysis methods.

[90] In SSA the largest scale at which the signal $X$ is analyzed in (10) is approximately $N \Delta t$, the length of the time series, and the largest period is the window width $M \Delta t$. A s a consequence, the EOF $\rho_{k}$ contain information from the whole time series, as in the Fourier transform.

[91] In order to define a local SSA, the SSA methodology was extended by using a time-frequency analysis within a running time window whose size $W$ is proportional to the order $M$ of the covariance matrix. $V$ arying $M$, and thus $W$ in proportion, a multiscale representation of the data is obtained. A local SSA is performed by sliding windows of length $W \leq N \Delta t$, centered on times $b=W / 2, \ldots, N \Delta t-W / 2$, along the time series. This method is useful when the local variability, assumed to be the sum of a trend, statistically significant variability, and noise, changes in time.

[92] A priori, the two scales $W$ and $M$ can vary independently, as long as $W$ is larger than $M \Delta t, W /(M \Delta t) \geq$ $\alpha>1$, and $\alpha$ is large enough [Vautard et al., 1992]. In the wavelet transform, however, the number of oscillations of the mother wavelet is fixed and independent of the scale (width) of the analyzing wavelet. In this spirit, Yiou et al. [2000] fixed the ratio $W / M=\alpha \Delta t$ and relied therewith on the oscillation property of the EOFs to provide a fixed number of zeroes for the data-adaptive "wavelet" $\rho_{k}$ of each local SSA analysis. They used $\alpha=$ 3 in most of their calculations, as well as only the one or two leading E OFs, $\rho_{1}$ and/or $\rho_{2}$, on each $W$ interval. This provides an analysis at a fixed scale $W$ (see Table 2). Sampling a set of $W$ values that follow a geometrical sequence, for instance, in powers of 2 or 3 , provides a multiscale analysis very similar to the wavelet transform.

[93] For a given position $b$ and fixed $W$, we thus obtain local EOFs that are the direct analogs of analyzing wavelet functions. The number of EOF oscillations increases roughly with order, and the zeroes of $\rho_{k+1}$ separate those of $\rho_{k}$; this emulates an important property of successive analyzing wavelets. The first EOF thus corresponds approximately to an analyzing wavelet function with a single extremum and no zero inside the window, for instance, the Gaussian or the "M exican hat"; such a basic wavelet is denoted by $\psi=\psi^{(0)}$ in Table 2. The second EOF has a single zero and is reminiscent of the first derivative of the Gaussian, denoted by $\psi^{(1)}$ in Table 2, and so on. Vautard and Ghil [1989] demonstrated this oscillation property of the EOFs for red noise in continuous time, and Allen [1992] did so for the same type of noise process in discrete time. A ppendix B of Yiou et al. [2000] provides a more general proof that is based on the concept of total positivity for lag-covariance matrices.

[94] For each $b$ and each EOF $\rho_{k}$, it is possible to obtain local PCs $\mathbf{A}_{k}$ and R Cs $\mathbf{R}_{k}$ (see equations (10) and (11)). The $k$ th PC at time $b$ is

$$
A_{k}^{b}(t)=\sum_{j=1}^{M} X(t+j-1) \rho_{k}^{b}(j),
$$


and the corresponding $\mathrm{RC}$ is

$$
R_{k}^{b}(t)=\frac{1}{M_{t}} \sum_{j=1}^{M} A_{k}^{b}(t-j) \rho_{k}^{b}(j),
$$

with $b-W / 2 \leq t \leq b+W / 2$. The crucial difference between this local version and global SSA is that the $\mathrm{R} C \mathrm{~S}$ are obtained here from local lag-covariance matrices. A s $b$ varies from $W / 2$ to $N \Delta t-W / 2$, this implies that the R Cs will be truncated near the edges of the time series.

[95] We thus see that the local SSA method provides simultaneous "wavelet transforms" of the data by a set of analyzing wavelet functions, corresponding to the $M$ different E OF s of the lag-covariance matrix. When $W=$ $\alpha M \Delta t$ is small, local SSA provides a small-scale analysis of the signal with a few distinct analyzing functions, i.e., a small subset of EO Fs indexed by $k$. This is reasonable, as there are not many possible structures at scales that approach the sampling timescale. On the other hand, at large scales, local SSA can also provide the simultaneous analysis by many different analyzing mother wavelet functions, $\left\{\rho_{k}: 1 \leq k \leq M\right\}$, and thus reflect the large complexity of the structures that is possible over the entire time series.

[96] The most important property of this local SSA analysis is that the analyzing functions are data adaptive. In other words, the shape of these analyzing functions is not imposed a priori, like in a wavelet analysis, but explicitly depends on the time series itself. For instance, an oscillatory behavior could be followed in a given time series by white or colored noise and then by deterministically intermittent behavior. These changes in behavior could indicate regime transitions that the system which generates the signal underwent while under observation. If so, an analyzing wavelet which is adapted to each section of the signal will definitely help follow such regime transitions in time.

[97] Yiou et al. [2000] performed multiscale SSA on the monthly SOI data for the years 1933-1996 (see section 2.2). The parameters were $\alpha=3$ and geometric scale increments of 2 . They computed, moreover, an "instantaneous" frequency by least squares fitting a sine wave to each local EOF of interest, as done in Monte Carlo SSA for the global EOFs. The instantaneous frequency can also be obtained from a complex wavelet transform [Delprat et al., 1992; Farge, 1992], by using information on the phase of the transform.

[98] The analysis of $\mathrm{Y}$ iou and colleagues did not reveal any evidence of self-similarity or fractality in the SOI. Instead, they find a preferred scale of variability between 3 and 5 years (not shown), which corresponds to ENSO's low-frequency mode (see section 2.2 and citations therein). The first two local EOFs are consistently paired and in phase quadrature, which shows that the nonlinear oscillation associated with this mode is robust and persists throughout the 60 -odd years being examined.
SOI 1933-96

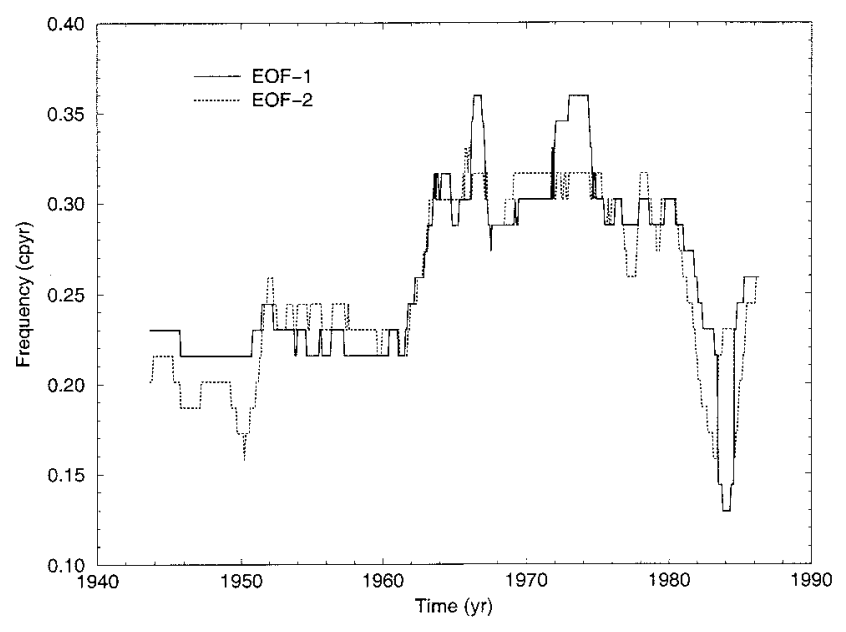

Figure 8. Instantaneous SOI frequencies, based on the two leading E O F s of multiscale SSA. These frequencies were computed independently for local EOFs 1 (solid curve) and 2 (dashed curve). The results agree well with each other and demonstrate a sharp jump in frequency between 1961 and 1962. Reprinted from Yiou et al. [2000] with permission of E Isevier Science.

[99] The computation of the instantaneous frequency in multiscale SSA allows one to detect an abrupt frequency shift of ENSO's low-frequency mode near 1960 (Figure 8). The characteristic periodicity goes from 57 months (between 1943 and 1961) to 39 months (between 1963 and 1980). A decrease in period in the early 1960s was observed already by Moron et al. [1998] in tropical Pacific sea surface temperatures, by using multichannel (global) SSA, and by Wang and Wang [1996] in a sea level pressure record at D arwin, using wavelet methods. Mann and Park [1996b, Figure 9] also observed a "pinch out" in the amplitude of the quasi-biennial oscillation in the 1960s.

[100] Moron et al. [1998] noticed, on the one hand, a change in the low-frequency mode's periodicity in the early 1960s by using multichannel SSA (see section 4.2) with different window widths ( $72 \leq M \leq 168$ months) on sea surface temperature fields for 1901-1994 (their Figure 2 and Table 4). On the other hand, these authors found that the trend of the sea surface temperatures in the tropical Pacific exhibited an increase from 1950 on (their Figure 4). They related this surface trend to a change in the parameters, such as the thermocline depth along the equator, of the coupled ocean-atmosphere oscillator responsible for E N SO [Ghil et al., 1991; Neelin et al., 1994, 1998].

[101] The frequency of a linear oscillator always changes smoothly as a function of the coefficients in the linear ODE that governs it. That is typically the case for nonlinear oscillators that depend on a single parameter as well [Stoker, 1950]. Two notable exceptions involve period doubling [Feigenbaum, 1978; Eckmann, 1981] and 
the D evil's staircase [Feigenbaum et al., 1982; Bak, 1986]. In both these routes to chaos, frequencies change abruptly as one parameter, in the former, or two, in the latter [Schuster, 1988], change gradually.

[102] Moron et al. [1998] hypothesized therefore that as one of the parameters of the EN SO oscillator crosses a threshold value in the early 1960s, the period of the seasonally forced climatic oscillator jumps from one step of the Devil's staircase to another. This would confirm an important aspect of recent theoretical work on ENSO's oscillatory regularity, as well as on the irregular occurrences of major warm and cold events [Chang et al., 1994; Jin et al., 1994, 1996; Tziperman et al., 1994].

[103] Yiou et al. [2000] compared the results in Figure 8 with those of Moron et al. [1998], using multichannel SSA (see section 4.2), and those of Wang and Wang [1996]. The latter authors used both the standard wavelet transform with a M orlet mother wavelet and Mallat and Zhang's [1993] waveform transform to analyze the $D$ arwin sea level pressures for 1872-1995. The frequency jump in Figure 8 is much sharper than when using either global SSA or wavelet-type methods (not shown here). It is also sharper than the changes in SOI variability described by Torrence and Compo [1998], who used both $M$ orlet and M exican-hat basis functions for their analysis. Multiscale SSA can thus provide sharper regime transitions in the evolution of a nonlinear system than either global SSA or a wavelet analysis that uses a fixed set of basis functions.

\section{SPECTRAL ANALYSIS METHODS}

\subsection{Generalities and Definitions}

[104] Both deterministic [Eckmann and Ruelle, 1985] and stochastic [Hannan, 1960] processes can, in principle, be characterized by a function of frequency $f$ (instead of time $t$ ). This function $S(f)$ is called the power spectrum in the engineering literature or the spectral density in the mathematical one (see section 1.1 for our use of the terms). Thus a very irregular motion possesses a smooth and continuous spectrum, which indicates that all frequencies in a given band are excited by such a process. On the other hand, a purely periodic or quasiperiodic process is described by a single line or a (finite) number of lines in the frequency domain. Between these two extremes, nonlinear deterministic but "chaotic" processes can have spectral peaks superimposed on a continuous and wiggly background [Ghil and Childress, 1987, section 12.6; Ghil and Jiang, 1998].

[105] In theory, for a spectral density $S(f)$ to exist and be well defined, the dynamics generating the time series has to be ergodic and allow the definition of an invariant measure, with respect to which the first and second moments of the generating process are computed as an ensemble average. The reason for the spectral theory of linear random processes being more familiar is simply that the construction of such an invariant measure is easier for such processes [Hannan, 1960; Priestley, 1981a]. In practice, the distinction between deterministically chaotic and truly random processes via spectral analysis can be as tricky as the attempted distinctions based on the dimension of the invariant set (see section 1.2 and citations therein). In both cases, the difficulty is due to the shortness and noisiness of climatic time series.

[106] Given the existence and invariance of an appropriate measure, the rigorous definition of the process's lag-correlation function involves a Lebesgue integral over that measure. $\mathrm{O} n$ the spectral side of the $\mathrm{W}$ iener$K$ hinchin equality (see equations (24) and (25) in the next section), one then has to use a Stieltjes integral, rather than the more familiar Riemann integral, with respect to the usual measure on $\mathbb{R}$ to compute the cumulative power $P(f)$ of the process over all frequencies between $-\infty$ and the given frequency $f$ [e.g., Hannan, 1960]. The spectrum is continuous at all points $f$ where the left and right limits of this integral are equal, i.e., $P(f-)=P(f+)$. At such points, $S(f)=d P / d f$. Where this is not the case, an upward jump in $P(f)$ occurs, i.e., $P(f+)=P(f-)+p(f)$, where $p(f)>0$. Such a discontinuity in $P(f)$ corresponds, loosely speaking, to a Dirac $\delta$ function in the "spectral density." The line spectrum is the sum of these $\delta$ functions that contributes to $S(f)$, along with the previously described continuous spectrum [e.g., Priestley, 1981a].

[107] In practice, spectral analysis methods attempt to estimate either the continuous part of the spectrum or the "lines" or both. The lines are often estimated from discrete and noisy data as more or less sharp "peaks." The estimation and dynamical interpretation of the latter, when present, are often more robust and easier to understand than the nature of the processes that might generate the broadband background, whether deterministic or stochastic.

[108] The numerical computation of the power spectrum of a random process is an ill-posed inverse problem [Jenkins and Watts, 1968; Thomson, 1982]. For example, a straightforward calculation of the discrete Fourier transform of a random time series, which has a continuous spectral density, will provide a spectral estimate whose variance is equal to the estimate itself [Jenkins and Watts, 1968; Box and Jenkins, 1970]. In the remainder of this section we outline three techniques to reduce this variance that are commonly used for the spectral analysis of climatic time series and point out their respective properties, advantages, and failings.

[109] E ach of the three techniques outlined in sections 3.2-3.4 below (Blackman-Tukey, maximum entropy, and multitaper) can provide confidence intervals for the estimates it produces. Still, these "error bars" are based on certain assumptions about the process generating the time series that is being analyzed. These assumptions are rarely, if ever, met in practice by the physical processes one wishes to examine. Therefore we highly recommend applying several independent techniques to a given time series before drawing any conclusion about its spectrum. 


\subsection{Classical Spectral Estimates}

[110] A generic problem of time series analysis is the finiteness of the time interval on which the series is known. Estimating the spectrum of $\{X(t): t=1, \ldots$, $N\}$ by a discrete Fourier transform yields its periodogram, which is the Fourier transform squared. D oing so corresponds to convolving the true spectrum with the spectrum of a boxcar function; this induces power leakage, due to the lobes of the Fourier transform of the boxcar. The resulting systematic distortion of the spectrum adds to the problem of the variance of the spectral estimate.

\subsubsection{The Periodogram and the Correlogram}

[111] The so-called periodogram estimate $\hat{S}_{X}(f)$ of $S(f)$ is given by

$$
\hat{S}_{X}(f)=\frac{\Delta t}{N}\left|\sum_{t=1}^{N} X(t) e^{-2 \pi i f t \Delta t}\right|^{2} ;
$$

the use of $i=\sqrt{-1}$ in this section should not be confused with the time index $i$ used in section 2 . The estimate $\hat{S}_{X}(f)$ is itself a random-valued function. Its mean leads to the correct $S_{X}$ as the length $N \Delta t$ of the sample series tends to infinity, but the standard deviation in this limit $N \rightarrow \infty$ is as large as the mean $S_{X}(f)$ being estimated [Hannan, 1960]. An estimate for which the variance does not tend to zero as the sample size tends to infinity is called statistically inconsistent. M oreover, for any finite $N$, the sample periodogram $\hat{S}_{X}$ is a biased estimate of $S_{X}$; that is, its mean over distinct realizations of the same process does not equal $S_{X}$ in general.

[112] Blackman and Tukey [1958] gave an alternative method to estimate the power spectrum of a given time series $\{X(t): t=1, \ldots, N\}$. Their method helps reduce the estimate's variance and bias and attenuate the leakage effects of the periodogram [Chatfield, 1984]. The method's starting point is the so-called W iener-K hinchin or Bochner-K hinchin-W iener theorem, which states that the spectral density $S_{X}$ is equal to the Fourier transform of the autocovariance function $\phi_{X}$ [Jenkins and Watts, 1968] (see also sections 1.1 and 3.1):

$$
\phi_{X}(k)=\mathscr{E}\{X(t+k) X(t)\},
$$

where $\mathscr{E}$ is the expectation operator.

[113] H ence the power spectrum $S_{X}(f)$ of $X(t)$ can be estimated by the Fourier transform $\tilde{S}_{X}(f)$ of an estimate $\hat{\phi}_{X}$ of the true $\phi_{X}$, called the correlogram:

$$
\tilde{S}_{X}(f)=\sum_{k=-(N-1)}^{N-1} \hat{\phi}_{X}(k) e^{-2 \pi i f k} .
$$

This estimate is also biased and inconsistent, as it stands. $M$ any methods have been devised to reduce the bias, variance, and leakage in the direct estimate (equation
(22)) or the indirect estimate (equation (24)). A succinct summary is provided by Ghil and Taricco [1997], and details are given in a number of textbooks and reference books [Chatfield, 1984; Kay, 1988].

[114] A simple way to reduce the bias and variance is to average the estimate over nonoverlapping bins in the frequency domain, in order to smooth the periodogram:

$$
\bar{S}_{X}\left(f_{k}\right)=\sum_{j=-M}^{M} g_{j} \hat{S}_{X}\left(f_{k-j}\right),
$$

where $\left\{f_{k}: k=1, \ldots, N_{f}\right\}$ is a discrete set of frequencies, with $N_{f} \leq N$, and $\left\{g_{j}\right\}$ is a set of $2 M+1$ smoothing weights, with $M<N / 2$. With well-chosen weights, the estimate $\bar{S}_{X}$ becomes consistent, i.e., its variance converges to 0 as $M$ and $N$ tend to infinity [Chatfield, 1984; Percival and Walden, 1993]. In (25) the choice of $M$ is dictated by a trade-off between frequency resolution (the larger $M$ the better) and the estimated variance, which is proportional to $M / N$ (hence the smaller $M$ the better) [Kay, 1988] (see al so the discussion of window width for SSA in section 2.2). Therefore a rule of thumb is to take $M$ no larger than $N / 5$ or $N / 10$, to avoid spurious results from high-variance estimates.

[115] The counterpart of this approach in the indirect estimation procedure is to use a lag window $\left\{W_{\mu}(k): k=-(N-1), \ldots, N-1\right\}$, with a smoothing parameter $\mu$. A n example of such a window is given in (28) below (see section 3.2.2). The power spectrum estimate then becomes

$$
\overline{\bar{S}}_{X}(f)=\sum_{k=-(N-1)}^{N-1} W_{\mu}(k) \hat{\phi}_{X}(k) e^{-2 \pi i f k} .
$$

With a careful choice of the shape of the window $\left\{W_{\mu}(k)\right\}$ for fixed $\mu$ and of the parameter value $\mu$, the estimate $\overline{\bar{S}}_{X}$ enjoys the same statistical properties as $\bar{S}_{X}$ in (25), i.e., consistency and lower bias. It turns out that the two procedures are formally equivalent and that a lag window $\left\{W_{\mu}(k)\right\}$ can be deduced from weights $\left\{g_{j}\right\}$ in (25), and vice versa [Percival and Walden, 1993]. Computationally, it is more efficient to calculate (23) and (26) than (22) and (25). Hence the indirect or correlogram method, associated with the names of Blackman and Tukey [1958], is most often the algorithm of choice.

[116] The windows traditionally have a compact support of length $m<N$, so that (26) becomes, with $\mu=$ $m$,

$$
\tilde{S}_{X}(f)=\sum_{k=-(m-1)}^{m-1} W_{m}(k) \hat{\phi}_{X}(k) e^{-2 \pi i f k} ;
$$

$m$ is called the truncation point or window width in this standard formula. Such lag windows are heuristically chosen as modified cosine functions, cubic functions, or tent functions [Chatfield, 1984]. Classical Blackman- 


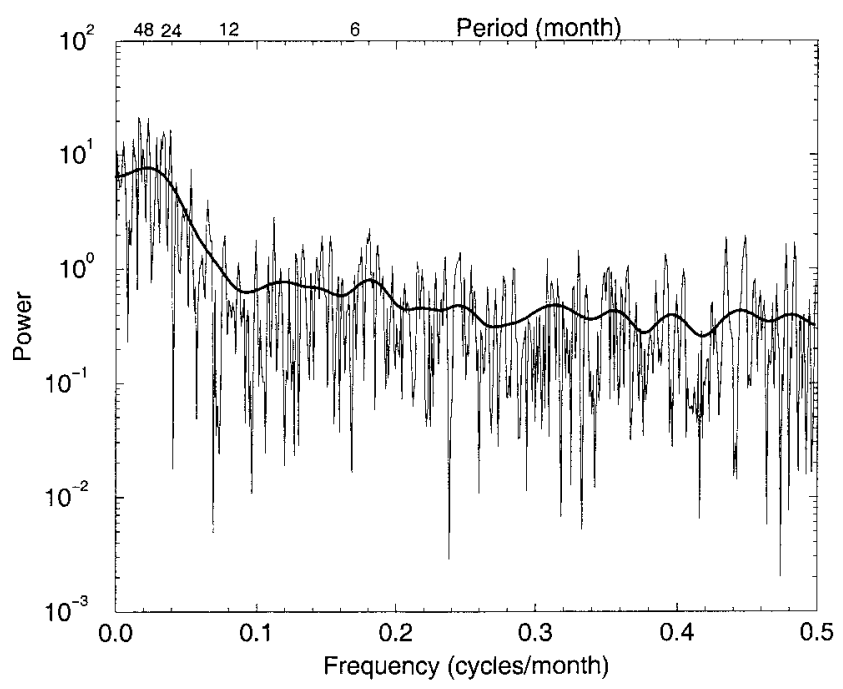

Figure 9. Classical spectral estimates of the SOI time series, from a raw correlogram (light curve) and lag-window estimate (bold curve), with a Bartlett window of width $m=40$.

Tukey estimation deals largely with the choice of window shape, often called "window carpentry," and of the window width $m$, often called "opening and closing" the window.

\subsubsection{Bias and Variance Reduction}

[117] We illustrate the variance reduction properties of the lag-window estimate in Figure 9 with a Bartlett window of varying width $m$. The Bartlett window is simply

$$
W_{m}(k)=1-\frac{|k|}{m},
$$

with $k=1, \ldots, m$. The smoothed estimate (bold curve) clearly has a much smaller variance than the raw periodogram estimate (light curve). Confidence levels relative to a pure red noise, or $A R(1)$ process, which has a spectral slope that behaves like $\left[1+(2 \pi f)^{2}\right]^{-1}$ (see equation (13)), can be calculated for each window shape [Jenkins and Watts, 1968], but the spectral resolution is generally poor if the number $N$ of data points, and hence $m$, is low.

[118] It turns out that the Blackman-Tukey, or windowed correlogram, method is quite efficient for estimating the continuous part of the spectrum, but is less useful for the detection of components of the signal that are purely sinusoidal or nearly so. The reason for this is twofold: the low resolution of this method and the fact that its estimated error bars are still essentially proportional to the estimated mean $\tilde{S}_{X}(f)$ at each frequency $f$. We shall see in section 3.4 how to choose, according to the multitaper method (MTM), a set of optimal tapers that maximize the resolution. M oreover, M TM also allows the formulation of statistical significance tests that are independent, in principle, of the amplitude of the sinusoidal component that is being estimated.

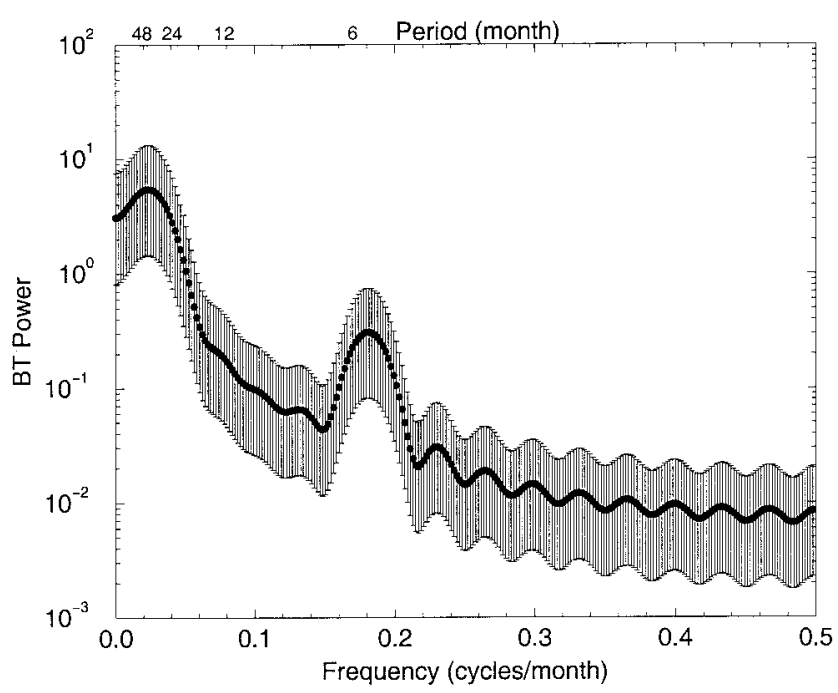

Figure 10. Blackman-Tukey correlogram estimate of the SSA -filtered SOI's spectral density. The embedding dimension for SSA is $M=60$, and RCs 1-4 and 10-11 were chosen as most significant. A Bartlett window of width $m=30$ was used for the spectral estimate of the prefiltered SOI. The error bars are based on a chi-square test and reach from the $2.5 \%$ to the $97.5 \%$ quantile.

[119] Vautard and Ghil [1989] showed the (data-adaptive) band-pass filtering effects of reconstructed components (RCS). In Figure 10 we illustrate the filtering properties of SSA on the SOI time series by removing the trend and "noise" components with SSA and computing the smoothed correlogram of the residual time series. This filter enhances the components with periodicities around 3.5 years and 5.6 months. These approximate periodicities will be confirmed and refined using the maximum entropy method (MEM) and M TM in the next two sections. Even with this SSA prefiltering, though, the lag-windowed correlogram is not able to distinguish a quasi-biennial from a quasi-quadrennial peak, as the MEM and MTM are able to do.

\subsection{Maximum Entropy Method (MEM)}

[120] This method is based on approximating the time series under study by a linear A R process (equation (1)) of order $M, \mathrm{AR}(M)$. It thus performs best when estimating line frequencies for a time series that is actually generated by such a process. Details are given by Burg [1967] and Childers [1978].

[121] Given a time series $\{X(t): t=1, \ldots, N\}$ that is assumed to be generated by a wide-sense stationary process with zero mean and variance $\sigma^{2}, M^{\prime}+1$ estimated autocovariance coefficients $\left\{\hat{\phi}_{X}(j): j=0, \ldots\right.$, $\left.M^{\prime}\right\}$ are computed from it:

$$
\hat{\phi}_{X}(j)=\frac{1}{N+1-j} \sum_{t=1}^{N-j} X(t) X(t+j) .
$$




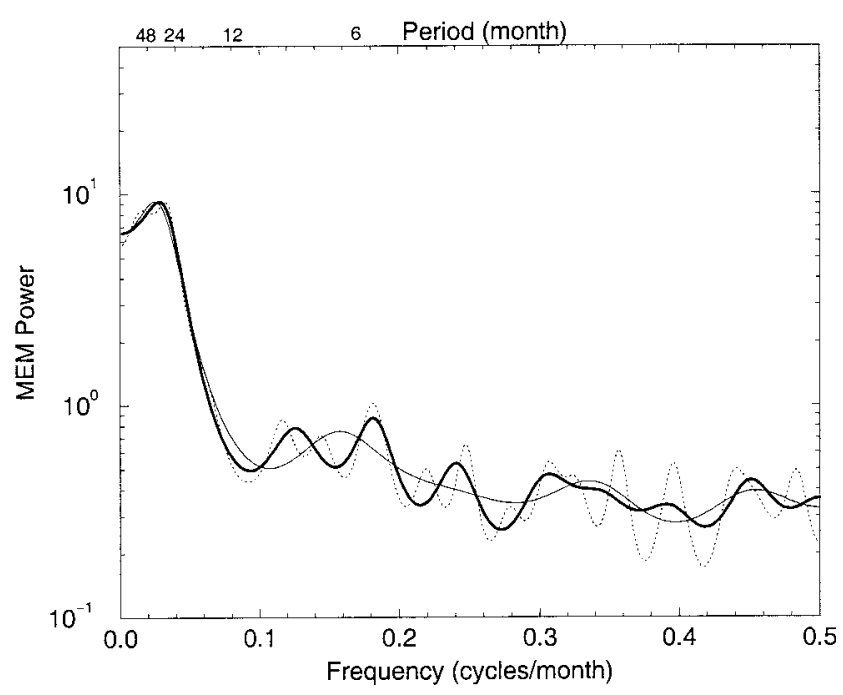

Figure 11. Spectral estimates of the SOI time series by the maximum entropy method (MEM). The autocorrelation orders are $M^{\prime}=10,20$, and 40 (light, bold, and dashed curves, respectively). The $A$ kaike information content (AIC) criterion [Haykin and Kessler, 1983] predicts an order of $M^{\prime} \approx 10$, which is obviously too low.

In the absence of prior knowledge about the process that generates the time series $X(t), M^{\prime}$ is arbitrary and has to be optimized. The purpose of evaluating (29) is to determine the spectral density $\hat{S}_{X}$ that is associated with the most random, or least predictable, process that has the same autocovariance coefficients $\hat{\phi}$. In terms of information theory [Shannon, 1949], this corresponds to the concept of maximal entropy, hence the name of the method.

[122] In practice, one obtains estimates $\left\{\hat{a}_{j}: j=\right.$ $\left.0, \ldots, M^{\prime}\right\}$ of the regression coefficients $a_{j}: j=0, \ldots$, $M$ \} from the time series $X(t)$ by assuming that the latter is generated by an A R $(M)$ process and that its order $M$ equals $M^{\prime}$. The autocorrelation coefficients $\hat{\phi}_{X}(j)$ are computed according to (29) and used to form the same Toeplitz matrix $\mathbf{C}_{X}$ as in SSA [Vautard and Ghil, 1989; Penland et al., 1991; Vautard et al., 1992]. This matrix is then inverted using standard numerical schemes [Press et $a l ., 1988]$ to yield the estimated $\left\{\hat{a}_{j}\right\}$. The spectral density $S_{X}$ of the true AR process with coefficients $\left\{a_{j}: j=\right.$ $0 \ldots, M\}$ is given by

$$
S_{X}(f)=\frac{a_{0}}{\left|1-\sum_{j=1}^{M} a_{j} e^{2 \pi i j f}\right|^{2}},
$$

where $a_{0}=\sigma^{2}$ is the variance of the residual noise $\xi$ in (1). Therefore the knowledge of the coefficients $\left\{\hat{a}_{j}: j=\right.$ $\left.0, \ldots, M^{\prime}\right\}$, determined from the time series $X(t)$, also yields an estimate $\hat{S}_{X}$ of the power spectrum.

[123] A $n$ example of MEM estimates is given for the SOI time series in Figure 11, using a number of lags $M^{\prime}$ $=10,20$, and 40 . It is clear that the number of peaks

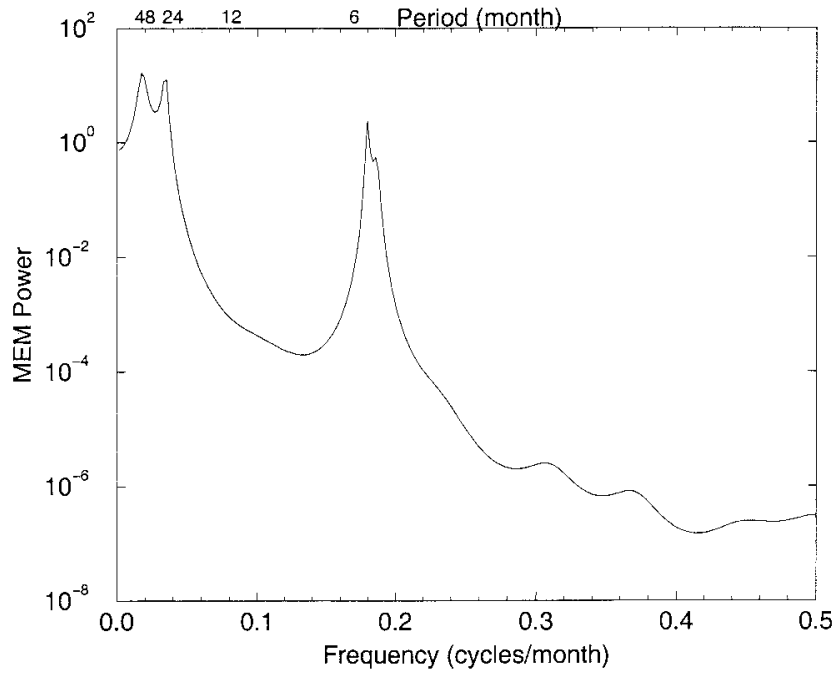

Figure 12. MEM analysis of the SOI time series after SSA prefiltering. The autoregression order used is $M^{\prime}=20$. The SSA window width is $M=60$, and RCs 1-4 and 10-11 were chosen, as in Figure 10.

increases with $M^{\prime}$. Two distinct interannual peaks appear only for $M^{\prime}=40$. This separation between a quasi-biennial and a low-frequency peak is accompanied, unfortunately, by many spurious peaks at higher frequencies.

[124] In general, if the time series is not stationary or otherwise not well approximated by an AR process (e.g., if it is generated by nonlinear dynamics), it is necessary to exercise great care in applying MEM. In such cases, cross testing with the application of other techniques is especially important.

[125] As the number of peaks in a MEM spectrum increases with $M^{\prime}$, regardless of the spectral content of the time series, an upper bound for $M^{\prime}$ is generally taken as $N / 2$. H euristic criteria have been devised to refine the choice of a reasonable $M^{\prime}$ [Haykin and Kessler, 1983; Benoist, 1986], based on minimizing the residual of a least squares fit between the A R approximation and the original time series [Akaike, 1969, 1974; Haykin and Kessler, 1983]. Such "information-content" criteria, however, often tend to either underestimate [Benoist, 1986] or overestimate [Penland et al., 1991] the order of regression of a time series, depending on its intrinsic characteristics.

[126] The effects of the $S / N$ enhancement performed by SSA decomposition (see section 2) on MEM analysis are illustrated in Figure 12, where the noise components identified by SSA were filtered out prior to MEM analysis. In this example, the power spectrum is much smoother than in Figure 11. The regression order $M^{\prime}=$ 20 suffices to separate a quasi-biennial peak at about 2.4 years from a quasi-quadrennial peak at roughly 4.8 years, while no spurious peaks appear at high frequencies. By contrast, Blackman-Tukey spectra of the same time series (see Figure 10 and Rasmusson et al. [1990]) 
fail to separate these two peaks with sufficient statistical confidence.

[127] The subannual peak at 5.6 months, which is associated with the EOFs $10-11$, is also much sharper here than in Figure 10. It can be distinguished therefore reliably from the known semiannual peak of tropical variability; in fact, the latter had been removed from the time series along with the seasonal cycle. A coupledmode solution with spatial features that resemble an oceanic K elvin wave and a period close to 6 months has been obtained in a hybrid ocean-atmosphere model with no seasonal cycle by Neelin [1990]. It provides a plausible physical mechanism for our 5.6-month peak.

\subsection{Multitaper Method (MTM)}

[128] This method provides useful tools for the spectral estimation [Thomson, 1982; Percival and Walden, 1993] and signal reconstruction [e.g., Park, 1992] of a time series whose spectrum may contain both broadband and line components. Like the classical periodogram and correlogram methods of section 3.2, M TM is nonparametric, since it does not use an a priori, parameterdependent model of the process that generated the time series under analysis, like M EM does. M TM reduces the variance of spectral estimates by using a small set of tapers [Thomson, 1982; Percival and Walden, 1993] rather than the unique data taper or spectral window used by the classical methods. The data are premultiplied by orthogonal tapers constructed to minimize the spectral leakage due to the finite length of the time series, and a set of independent estimates of the power spectrum is computed.

[129] The optimal tapers or "eigentapers" belong to a family of functions known as discrete prolate spheroidal sequences (DPSS). They are defined as the eigenvectors of a suitable $R$ ayleigh- $R$ itz minimization problem and were extensively studied by Slepian [1978]. More precisely, the tapers are the discrete set of eigenfunctions that solve the variational problem of minimizing leakage outside of a frequency band with half bandwidth equal to $p f_{R}$, where $f_{R}=1 /(N \Delta t)$ is the $\mathrm{R}$ ayleigh frequency, $\Delta t$ is the sampling interval, and $p$ is a suitably chosen integer. Because the windowing functions or eigentapers are the specific solution to an appropriate variational problem, this method is less heuristic than traditional nonparametric techniques (see section 3.2 as well as Box and Jenkins [1970] and Jenkins and Watts [1968]). A veraging over the (small) ensemble of spectra obtained by this procedure yields a better and more stable estimate, i.e., one with lower variance, than do single-taper methods [Thomson, 1990a].

[130] Detailed algorithms for the calculation of the eigentapers are readily available [Thomson, 1982; Percival and Walden, 1993]. In practice, only the first $2 p-$ 1 tapers provide usefully small spectral leakage [Slepian, 1978; Thomson, 1982; Park et al., 1987]. Thus the number $K$ of tapers used should be less than $2 p-1$ in any application of MTM .

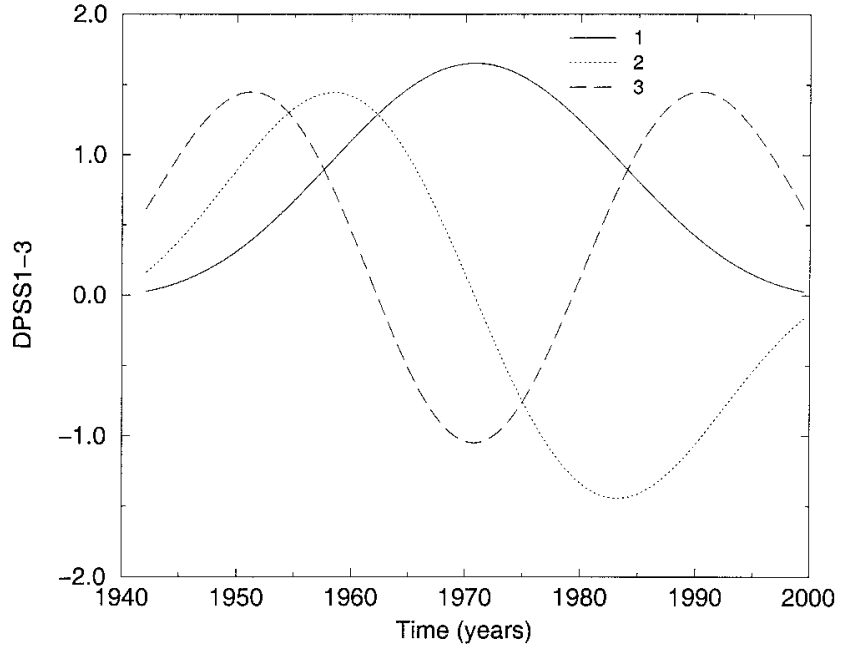

Figure 13. The first $K=3$ eigentapers for the case $p=2$, computed for the length $N=690$ of the SOI time series in Figure 2. Note that the third eigentaper or discrete prolate spheroidal sequence (DPSS) is the least leakage resistant since its values near the boundaries $t=0$ and $t=N \Delta t$ of the sampling interval are no longer negligible, like those of the first two DPSSs. The eigentapers shown have been normalized to unit power.

[131] The choice of the bandwidth $2 p f_{R}$ and number of tapers $K$ thus represents the classical trade-off between spectral resolution and the stability or variance reduction properties of the spectral estimate [Thomson, 1982]. The case $p=1$ and $K=1$ is simply the singletapered discrete Fourier transform (DFT) of Blackman and Tukey [1958]. For instrumental climate records, with a typical length of a few hundred points, the choice $p=$ 2 and $K=3$ offers a good compromise between the required frequency resolution for resolving distinct climate signals (e.g., ENSO and decadal-scale variability) and the benefit of multiple spectral degrees of freedom, i.e., of reduced variance [e.g., Mann and Park, 1993]. L onger data sets permit the use of a greater number $K$ of tapers while maintaining a desired frequency resolution. The optimal choice of $p$ and $K$ depends, in general, on the length and other properties of the time series under study.

[132] We show in Figure 13 the $K=3$ leakageresistant Slepian tapers for bandwidth parameter $p=2$. In this example, the effective half bandwidth is $2 f_{R}$ for the spectral estimate centered at any particular frequency $f_{0}$. This half bandwidth is a measure of spectral resolution and equals, in the present case, twice that of an unsmoothed DFT. Thus a spectral estimate at the 5 -year periodicity of $f=0.2$ cycle/yr for a time series with $N=690$ months (57.5 years) averages the raw periodogram over the frequency interval between $f \approx$ 0.165 and $f \approx 0.235$ cycle/yr. The variance of the spectral estimate at $f_{0}$ is decreased, on the other hand, threefold by averaging the three independent eigenspectra. This variance versus resolution trade-off is clearly 
superior to conventional periodogram smoothing. The process of MTM spectral estimation is described in greater detail in section 3.4.1.

[133] Because of the improvements in its spectral estimation properties over the classical methods of section 3.2, the MTM method has been widely applied to problems in geophysical signal analysis, including analyses of instrumental data on the atmosphere and oceans [Kuo et al., 1990; Ghil and Vautard, 1991; Mann and Park, 1993, 1994, 1996a, 1996b; Lall and Mann, 1995; Mann et al., 1995b; Thomson, 1995], paleoclimate proxy data [Chappellaz et al., 1990; Thomson, 1990a, 1990b; Berger et al., 1991; Mann et al., 1995a; Mann and Lees, 1996; Mommersteeg et al., 1995; Park and Maasch, 1993; Yiou et al., 1991, 1994, 1995, 1997], geochemical tracer data [Koch and Mann, 1996], and seismological data [Park et al., 1987; Lees, 1995]. Time-frequency "evolutive" analyses based on moving-window adaptations of M TM have also been applied to paleoclimatic records and model simulations [Yiou et al., 1991; Birchfield and Ghil, 1993; Mann et al., 1995a; Mann and Park, 1996b].

\subsubsection{Spectral Estimation}

[134] MTM can provide estimates of both the line components and the continuous background of the spectrum. Once the tapers $w_{k}(t)$ are computed for a chosen frequency bandwidth, the total power spectrum $S_{X}$ can be estimated by averaging the individual spectra given by each tapered version of the data set. We call $\hat{S}_{k}(f) \equiv$ $\left|Y_{k}(f)\right|^{2}$ the $k$ th eigenspectrum estimate, where $Y_{k}$ is the discrete Fourier transform (DFT) of $\left\{X(t) w_{k}(t): t=\right.$ $1, \ldots, N\}$. The high-resolution multitaper spectrum is a weighted sum of the $K$ eigenspectra,

$$
S_{r}(f)=\frac{\sum_{k=1}^{K} \mu_{k}\left|Y_{k}(f)\right|^{2}}{\sum_{k=1}^{K} \mu_{k}} ;
$$

for the choice of weights $\mu_{k}$ and other details, see Percival and Walden [1993].

[135] This spectral estimate's frequency resolution is $\pm p f_{R}$, which means that line components will actually be detected as peaks or bumps of width $2 p f_{R}$. The situation is thus similar, in principle, to that for the classical spectral estimate of section 3.2, except that the peaks can be identified with a higher resolution and greater confidence in M TM. For a white-noise process, or even one that has a locally flat spectrum near the line of interest, the high-resolution spectrum is chi-square distributed with $2 K$ degrees of freedom [Thomson, 1982].

[136] The relative weights on the contributions from each of the $K$ eigenspectra can be adjusted further to obtain a more leakage-resistant spectral estimate, termed the adaptively weighted multitaper spectrum,

$$
S_{w}(f)=\frac{\sum_{k=1}^{K} b_{k}^{2}(f) \mu_{k}\left|Y_{k}(f)\right|^{2}}{\sum_{k=1}^{K} b_{k}^{2}(f) \mu_{k}} .
$$

The weighting functions $b_{k}(f)$ further guard against broadband leakage for a "warm-colored" process (see section 2.3) that is locally white, i.e., that has a fairly flat spectrum in the frequency range of interest. The adaptive spectrum estimate has an effective number of degrees of freedom $v$ that generally departs only slightly from the nominal value $2 K$ of the high-resolution multitaper spectrum [Thomson, 1982].

[137] The purpose of harmonic analysis is to determine the line components in the spectrum that correspond to a purely periodic or multiply periodic signal in terms of their frequency, amplitude, and phase. The Fourier transform of a clean periodic signal in continuous time and of infinite length yields a D irac function at the frequency of the signal, namely, a line (or peak of zero width) with infinite magnitude. As described in section 3.1, it is the jump in the cumulative power at that frequency that is proportional to the periodic signal's amplitude squared.

[138] A spectral estimate based on the methods discussed so far gives indirect information on such a signal's amplitude at all frequencies. For a periodic signal sampled at discrete times over a finite time interval, the area under the peak centered at its true frequency is proportional to the signal's amplitude squared, while the peak's width is, roughly speaking, inversely proportional to the length $N$ of the time series. The area under the peak is nearly constant as $N$ changes, since the peak's height is proportional to $N$.

[139] $\mathrm{H}$ armonic analysis attempts to determine directly the (finite) amplitude of a (pure) line in the spectrum of a time series of finite length. We explain next how this is done within MTM. MacDonald [1989] described pure line estimation using the maximum likelihood approach for single-window periodogram estimators [Schuster, 1898; Whittle, 1952]. Foias et al. [1988] proved rigorous mathematical results on maximum likelihood estimation of sinusoids in noise of arbitrary color. The drawback of these single-window results is that they work well only when the $S / N$ ratio is fairly high. Thus SSA prefiltering, which enhances the S/N ratio, may help "classical" pure line estimation, as suggested by the results presented in sections 3.2 and 3.3 for standard peak detection. The MTM approach described below, on the other hand, can also ascertain the presence of pure sinusoids in a fairly high noise background.

[140] A ssume the time series $X(t)$ is the sum of a sinusoid of frequency $f_{0}$ and amplitude $B$, plus a "noise" $\eta(t)$ which is the sum of other sinusoids and white noise. One can then write 


$$
X(t)=B e^{2 \pi i f o t}+\eta(t) .
$$

If $\left\{w_{k}(t): k=0, \ldots, K-1\right\}$ are the first $K$ eigentapers and $U_{k}(f)$ is the DFT of $w_{k}$, a least squares fit in the frequency domain yields an estimate $\hat{B}$ of the amplitude $B$ :

$$
\hat{B}\left(f_{0}\right)=\frac{\sum_{k=1}^{K} U_{k}^{*}(0) Y_{k}\left(f_{0}\right)}{\sum_{k=1}^{K}\left|U_{k}(0)\right|^{2}},
$$

where the asterisk denotes complex conjugation.

[141] A statistical confidence interval for $\hat{B}$ can be given by a Fisher-Snedecor test of $F$ test [Kendall and Stuart, 1977]. This test is based roughly on the ratio of the variance captured by the filtered portion of the time series $X(t)$, using $K$ eigentapers, to the residual variance. By expanding the variance of the model, one finds that it is the sum of two terms,

$$
\begin{gathered}
\theta=\left|\hat{B}\left(f_{0}\right)\right|^{2} \sum_{k=1}^{K}\left|U_{k}(0)\right|^{2} \\
\psi=\sum_{k=1}^{K}\left|Y_{k}\left(f_{0}\right)-\hat{B}\left(f_{0}\right) U_{k}(0)\right|^{2},
\end{gathered}
$$

that are the "explained" and "unexplained" contributions, respectively, to the variance.

[142] The random variable $F(f)$ of the unknown frequency $f$ is defined by

$$
F(f)=(K-1) \frac{\theta}{\psi} .
$$

If the time series $X(t)$ were a pure white-noise realization, $F$ would obey a Fisher-Snedecor law with 2 and $2 K-2$ degrees of freedom. One can interpret its numerical value for given data by assuming that $B=0$, i.e., that $X(t)$ is white, and trying to reject the whitenoise null hypothesis. In practice, the spectrum need only be "locally white" in the sense that the $K$ eigenspectra which describe the local characteristics of the spectrum should be distributed as they would be for white noise [Thomson, 1982].

[143] This harmonic analysis application of M TM is able to detect low-amplitude harmonic oscillations in a relatively short time series with a high degree of statistical significance or to reject a large amplitude if it failed the $F$ test, because the $F$ value $F(f)$ does not depend, to first order, on the magnitude of $\hat{B}(f)$. This feature is an important advantage of M TM over the classical methods of section 3.2, in which the error bars scale with the amplitude of a peak [e.g., Jenkins and Watts, 1968].

[144] The key assumption in this harmonic analysis approach, however, is that the time series is produced by a process that consists of a finite superposition of separate, purely periodic, fixed-amplitude components and white or, at least, locally white noise. If not, a continuous spectrum, in the case of a colored noise or a chaotic system, will be broken down into spurious lines with arbitrary frequencies and possibly high $F$ values. In essence, the above procedure assumes that the signal is represented by lines in the spectrum corresponding to phase-coherent harmonic oscillations, while the noise is represented by the continuous component of the spectrum.

[145] In geophysical applications, signals are most often associated with narrowband variability that is not strictly harmonic, i.e., sinusoidal, or even purely periodic. Truly harmonic signals are, in fact, rarely detected in climatic and most other geophysical time series [Ghil and Childress, 1987; Park, 1992]. This is often because the period, amplitude, and phase of a limit cycle like that illustrated in Figure 1 are typically each a function of slowly changing parameters. Such signals are well captured by the SSA's pairs of eigenelements (see section 2.2). To analyze the precise spectral content of an SSA oscillatory pair, however, requires the application of additional spectral estimation tools (see sections 3.2 and 3.3).

[146] The complex nature of geophysical signals and noise outlined in the preceding paragraph has motivated Mann and Lees [1996] to modify the conventional M TM approach to line estimation. Their algorithm combines the harmonic signal detection procedure described above with a criterion for detecting significant narrowband, "quasi-oscillatory" signals which may exhibit phase and amplitude modulation as well as intermittently oscillatory behavior. It thus provides for the detection of both harmonic and narrowband signals while making use of a robust estimate of the background noise.

[147] Mann and Lees [1996] retain the pure line test of the traditional MTM procedure, as given by (34)-(37). A Il peaks, however, whether purely harmonic or narrowband, are tested for significance relative to the null hypothesis of a red-noise background. The spectral density of this background noise is assumed to be given by (13)-(15), and the parameters $S_{0}$ and $r$, the noise process's variance and its lag-one autocorrelation, are estimated empirically from the data.

[148] Discrimination against a red-noise background is particularly important in climate studies, where the system under investigation always contains longer timescales than those of immediate interest. This leads to greater power at lower frequencies and greater likelihood of prominent peaks in the spectrum there, even in the absence of any signals [e.g., Hasselmann, 1976; Mitchell, 1976]. Thomson [1990a] and Thomson and Chave [1990] have developed therefore quadratic inverse spectrum estimates aimed more particularly at the shape and properties of continuous spectra and cross spectra. 
[149] Mann and Lees [1996] provide a robust estimate of the spectral background (equation (13)) by minimizing, as a function of $r$, the misfit between an analytical $A R$ (1) red-noise spectrum and the adaptively weighted multitaper spectrum convolved with a median smoother. The median smoothing operation insures that the globally estimated noise background is insensitive to "outliers." These outliers may be due to peaks associated with significant signals or to spurious ones. The median smoother guards against inflated estimates of noise variance and noise autocorrelation; the latter arise in a conventional Box-J enkins approach due to the contamination of noise parameters by contributions from the signal, for example, from a significant trend or oscillatory component of the series. A median smoothing width of $\Delta f=\min \left(f_{N} / 4,2 p f_{R}\right)$ is a good compromise between describing the full variation of the background spectrum over the Nyquist interval, on the one hand, and insensitivity to narrow spectral features, on the other.

[150] Significance levels for harmonic or narrowband spectral features relative to the estimated noise background can be determined from the appropriate quantiles of the chi-square distribution, by assuming that the spectrum is distributed with $v=2 K$ degrees of freedom [Mann and Lees, 1996]. A reshaped spectrum is determined in which the contributions from harmonic signals are removed [Thomson, 1982], based on their passing a significance threshold for the $F$ test on the variance ratio, as described above. In this way, noise background, harmonic, and narrowband signals are isolated in two steps. The harmonic peak detection procedure provides information as to whether the signals are best approximated as harmonic or narrowband, i.e., as phase-coherent sinusoidal oscillations or as amplitude-and-phasemodulated, and possibly intermittent, oscillations. In either case, they must be found to be significant relative to a specified noise hypothesis, such as that of red noise (equation (13)) used above.

[151] To illustrate the revised MTM procedure of Mann and Lees [1996], we apply the approach to the SO I series discussed earlier. Consistent with the SSA -M EM results of section 3.3 (see Figure 12 ), the MTM analysis in Figure 14 recognizes two highly significant interannual peaks, one centered at $f=0.18$ cycle/yr (roughly a 5.5-year period) and another centered at $f=0.41$ cycle/yr (roughly a 2.5-year period).

[152] These two signals are significant well above the 99\% level: For $p=2$ and $N=690$ monthly samples, there are about seven statistically independent bandwidths in the spectral estimate within the subannual band ( $f<0.5$ cycle/yr); hence not even one interannual or lower-frequency peak is expected to arise by pure chance at the $99 \%$ level. A low-frequency variation that cannot be distinguished from a trend for the given data set is also isolated as significant at the $99 \%$ level relative to the estimated red-noise background.

[153] We associate the two interannual peaks with the low-frequency and quasi-biennial E NSO signals, respec-

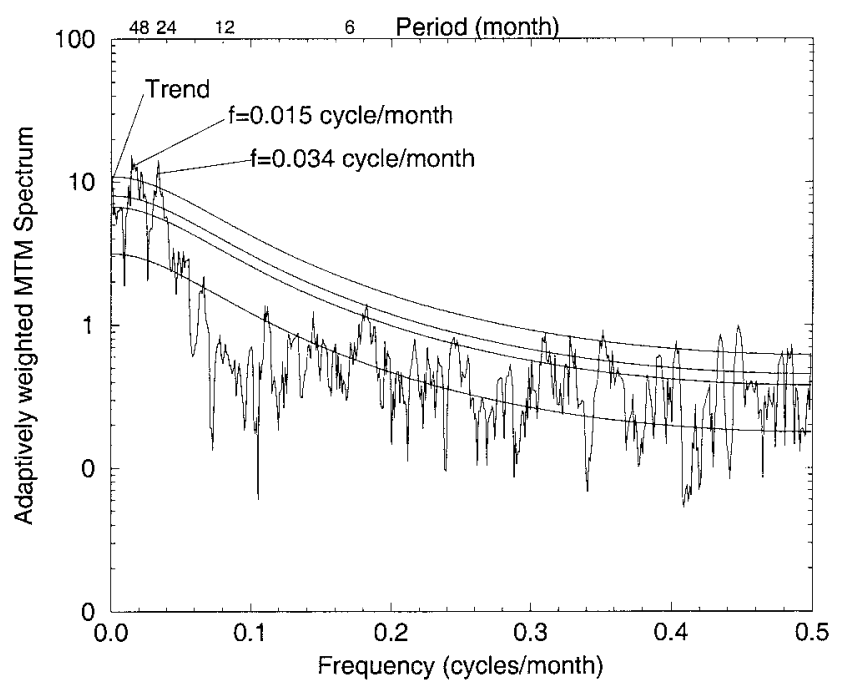

Figure 14. A daptively weighted MTM spectrum of the SOI time series. The estimated red-noise background and associated $90 \%, 95 \%$, and $99 \%$ significance levels are shown by the four smooth curves, in this order, from the lowest to the highest curve in the figure. Three signals with interannual and lower frequencies (the trend and ENSO's low-frequency and quasi-biennial signals) are significant at the $99 \%$ level. Several higher-frequency peaks appear also to be significant; see text for a more detailed assessment of their significance. The bandwidth parameter is $p=2$, and $K=3$ tapers were used.

tively, although a slight discrepancy exists with respect to the SSA-MEM results in Figure 12. The difference between the 2.5-year period here and the 2.4 years in Figure 12 is negligible. That between 5.5 years here and 4.8 years there is probably due to the relative shortness of the record and the width of the peak, both here and in Figure 12. SSA-M EM emphasizes the middle of the peak, while M TM selects a harmonic feature that might lie close to one of the peak's sides.

[154] It is interesting to note that the AR(1) model does not provide a good description of the noise background in a neighborhood of the spectrum surrounding $f=1.0$ cycle/yr (see also Figure 7 and its discussion in section 2.3). This discrepancy could be due, at least in part, to the prior removal of the seasonal cycle (see also Thomson [1995] and section 2.3). A way from $f=1.0$ cycle/yr the robustly estimated red-noise spectrum provides a good visual fit to the data set's spectral background. Mann and Lees's [1996] noise background estimation procedure thus appears to be fairly insensitive to the anomalous behavior of the spectrum near the annual cycle.

[155] H igher-frequency peaks between $f=0.3$ cycle/ month and $f=0.5$ cycle/month (periods of 2-3 months) may be associated with intraseasonal oscillations in the tropical atmosphere but raise some concerns through their large number and relatively poor match with results obtained using other atmospheric data sets. The estimate of the noise background in this intraseasonal band is probably lowered unduly by the attempt to fit a single 

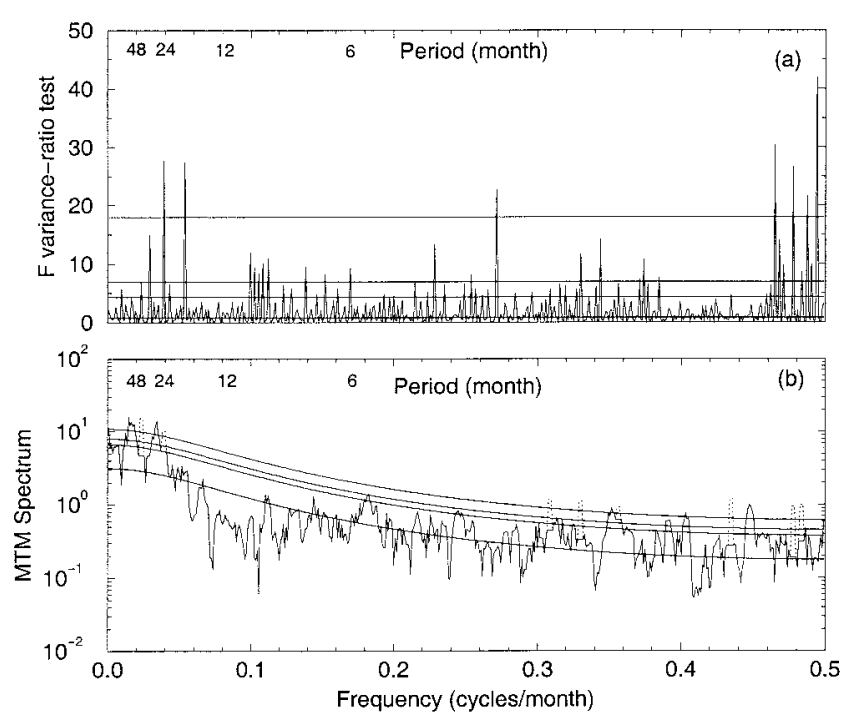

Figure 15. Comparison between MTM spectra obtained by harmonic analysis test and adaptively weighted estimation against red-noise background. Shown are (a) harmonic signal (variance ratio) $F$ test with median, $90 \%, 95 \%$, and $99 \%$ significance levels indicated and (b) reshaped versus unreshaped adaptively weighted MTM spectrum based on $p=2$ and $K=3$, and a $90 \% F$ test significance criterion for reshaping. The dashed line in Figure $15 \mathrm{~b}$ represents the discrepancy between reshaped spectrum (solid curve) and the unreshaped one; it thus provides a measure of the portion of the M TM spectrum associated with harmonic features. Significance levels for the reshaped spectrum are as in Figure 14.

red-noise spectrum to both this band and the interannual one. Gilman et al. [1963] al ready had recognized that multiple decorrelation timescales may characterize certain atmospheric and climate phenomena. Thus one might be better off in a case like this, when applying M TM , to use a more complex null hypothesis for significance testing. Such a hypothesis of a fairly general colored-noise background is available in the SSA-M TM Toolkit (http://www.atmos.ucla.edu/tcd/ssa), where it is described as "locally white." This composite noise model, however, has less of a physical justification than the straightforward red noise of (13)-(16) and raises questions of parsimony in estimating its parameters.

[156] To better understand the distinction between the inferences from the conventional MTM procedure of Thomson [1982] and the revised procedure of Mann and Lees [1996], we compare the results of the two in Figure 15. The $F$ test criterion for harmonic signals in Figure 15 a yields seven peaks at the $99 \%$ confidence level and 27 peaks at the $95 \%$ confidence level. M any of these peaks are associated with very weak power in the spectrum. Note that the harmonic peak test has the higher $\mathrm{R}$ ayleigh frequency resolution $\pm f_{R}$, and not the broader bandwidth $\pm p f_{R}$ of the adaptive multitaper spectral estimate (equation (32)). In a series of $N=690$ data points we would expect about seven and 35 peaks at the 99\% and $95 \%$ confidence levels, respectively, from chance alone. This does not differ significantly from the $F$ test results for the time series in Figure 2. The harmonic analysis approach alone therefore does not distinguish any significant features from random noise in our SOI data.

[157] The results of this approach are nonetheless useful in the reshaping procedure used by Mann and Lees [1996]. Figure 15b also shows the reshaped (solid curve) and adaptively weighted (dashed curve) multitaper spectrum along with the significance levels relative to the robustly estimated red-noise background. The dashed peaks satisfy the harmonic detection test at the $90 \%$ level and are also significant relative to red noise at the same level. There are eight such peaks, including both a lower-frequency and a quasi-biennial ENSO peak. The inference in this case is that the two distinct ENSO-band signals are both significant well above the 99\% level against red noise; furthermore, each of these two narrowband signals is likely to contain a harmonic, phase-coherent oscillation with a $90 \%$ level of confidence. If a $99 \%$ threshold were required in the $F$ test for the reshaping procedure, neither of these two peaks would pass the harmonic test. N onetheless, they are still highly significant narrowband features of the spectrum. The true E NSO signal is almost certainly associated with amplitude, phase, and frequency modulation over time (see Jiang et al. [1995a] and Figures 6 and 8); such features are not appropriately modeled in the harmonic signal test alone.

\subsubsection{Signal Reconstruction}

[158] O nce significant peaks have been isolated in the spectrum, relative to the specified null hypothesis, the associated signals can be reconstructed in the time domain using the information from the multitaper decomposition. These reconstructions of oscillatory signals are analogous to the SSA reconstructed components (RCS) described in section 2.2, except that information from a frequency domain decomposition, rather than a lag domain decomposition, is used to reconstruct the partial signal of interest. As in the lag domain case of SSA, the reconstruction becomes more delicate near the end points of the time interval over which the data are provided.

[159] The reconstructed signal that corresponds to a peak centered at frequency $f_{0}$ is written in continuous time as

$$
\tilde{X}(t)=\Re\left\{A(t) e^{-2 \pi i f o t}\right\}
$$

or, for the discrete-time case at hand,

$$
\tilde{X}(n \Delta t)=\Re\left\{A_{n} e^{-2 \pi i f o n \Delta t}\right\} .
$$

We determine the envelope function $A(t)$ from a time domain inversion of the spectral domain information contained in the $K$ complex eigenspectra [Park, 1992; Park and Maasch, 1993; Mann and Park, 1994, 1996b], while $\Re$ is the real part of the Fourier term in braces. 


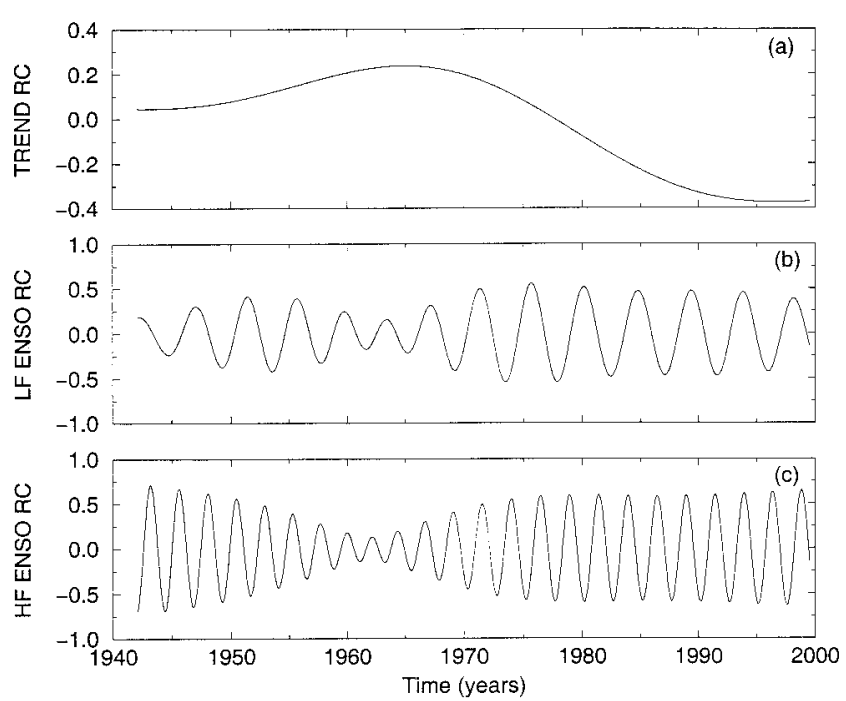

Figure 16. Multitaper reconstruction of the significant interannual components corresponding to (a) the trend, (b) the low-frequency band, and (c) the quasi-biennial band of E NSO . A minimum-misfit boundary condition was employed in the reconstructions, as described in the text. The parameters $p=$ 2 and $K=3$ were used in Figures 14 and 15.

The envelope $A(t)$ has $K$ complex degrees of freedom, and allows for phase and amplitude variations in the time reconstruction of the signal centered at frequency $f_{0}$. It thus represents a considerable refinement of the classical, single-taper complex demodulation method [Bloomfield, 1976; Hasan, 1983].

[160] The discrete time sequence describing the complex envelope $A_{n}$ is determined from a discrete inverse problem that uses the complex amplitudes of each of the $K$ eigenspectra and appropriate boundary conditions [Park, 1992; Park and Maasch, 1993]. The three lowestorder boundary constraints in this inversion involve minimizing the envelope $A_{n}$ itself or its slope near the end points, or maximizing its smoothness there. Supplementary information regarding the signal (see the discussion by Park [1992]) might favor any one of these three choices that involve the sequence $A_{n}$ and its first or second divided differences. The amplitude of the seasonal cycle in surface temperature, for example, is nearly constant in time, and a minimum-slope constraint is thus most appropriate [see Mann and Park, 1996b]. In the absence of such a priori information, one can obtain a nearly optimal reconstruction through seeking the weighted linear combination of these three constraints that minimizes the meansquare difference between the reconstructed signal and the raw data series [Mann and Park, 1996b].

[161] In Figure 16 we show the reconstruction, based on the latter recipe, of the $\mathrm{SO}$ I that corresponds to the three interannual signals that are significant at the $99 \%$ level in Figure 14: the trend, low-frequency, and quasibiennial components. The two oscillatory signals each exhibit roughly one (nondimensional, normalized) unit of peak-to-peak variation. The amplitude modulations of both oscillatory components, low-frequency (Figure

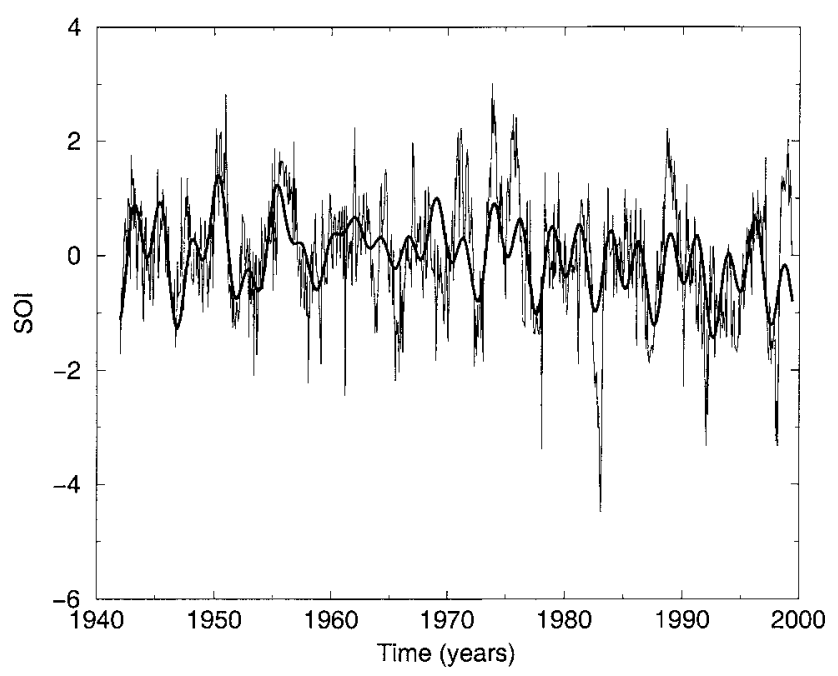

Figure 17. Sum of the three MTM reconstructed components (bold curve), along with the raw SOI series (light curve).

16b) and quasi-biennial (Figure 16c), are similar, with minimal amplitude in the early 1960s (compare with Figure 8 and its discussion in section 2.4).

[162] The long-term trend in the SOI (Figure 16a), while weak, still exhibits a peak-to-peak variation of about 0.5 units or one half of the amplitude of the two oscillatory signals. The oscillatory components of SOI are well known to be anticorrelated with sea surface temperatures in the eastern tropical Pacific. The trend in Figure 16a also seems to be anticorrelated with the temperature trend for the equatorial Pacific shown in Figure 4b of Moron et al. [1998], although the number of degrees of freedom in these two trends does not allow us to establish the statistical significance of this anticorrelation. The filtered R C -5 in Figure $6 \mathrm{~b}$ shares the overall features of the reconstructed trend in Figure 16a, although it also displays some interdecadal variability not present in the latter.

[163] In Figure 17 we plot the partial reconstruction of SOI (bold curve) obtained by summing the trend and two oscillatory components shown separately in Figure 16. This reconstruction captures $28 \%$ of the total variance in the raw monthly data (light curve) versus the $43 \%$ captured by R Cs 1- 4 in Figure 6a. The overall match with strong and moderate EI Niños and La Niñas is also fairly good, although somewhat less compelling than in section 2.2. The MTM trend in Figure 16a, on the other hand, seems to provide a cleaner picture of the SOI's nonoscillatory evolution over the latter half of the twentieth century than the SSA trend in Figure 6b. Both SSA and MTM reconstructions therefore have much to contribute to our description and understanding of climate variability.

\section{MULTIVARIATE METHODS}

[164] So far, we have discussed the spectral analysis of a single, scalar time series. In many geophysical applica- 
tions, and to a lesser extent in astrophysics and space physics, one is presented with a sequence of maps or other samples of a vector-valued process. The $L$ components at each discrete time may be values of a field at points of a regular or irregular grid, in one, two, or three space dimensions, or values of the field's projections onto certain (spatial) basis functions imposed by the geometry of the domain. It is useful to think of the values of such a component at successive times as a (scalar) time series, and it is common to refer to each of these time series as a channel. The methods discussed in this section are thus referred to sometimes as multichannel. It is also common in the statistical literature to refer to a scalar time series as univariate and to a vector time series as multivariate.

[165] M ost of the univariate methods of sections 2 and 3 (SSA, wavelets, M E M , and M TM ) have been extended to multivariate time series. The computation of cross correlations and cross spectra by classical methods is presented by Hannan [1970] and Priestley [1981b], among others. Mann and Park [1999] provide an up-todate review of multivariate MTM methods and their applications. There exists extensive literature on applying wavelets to the evolution of turbulent flow fields [Farge, 1992] and related multivariate problems [Torrence and Compo, 1998]. For the sake of brevity, we shall restrict ourselves therefore to a succinct presentation of multivariate MEM and SSA.

\subsection{Principal Oscillation Patterns (POPs)}

[166] In principle, one can extend MEM to multivariate time series via the inverse modeling of vector-valued A R processes [Ooms, 1988]. In practice, if both the number $L$ of channels and the order $M$ of the AR model are high, the issues of computational and statistical stability of the $L M$ coefficients involved become rapidly prohibitive. Thus it is only vector-valued AR(1) processes that have been widely applied to geophysical problems under the name of principal oscillation patterns (POPs) [Hasselmann, 1988; Von Storch et al., 1988] or empirical normal modes (ENMS) [Penland, 1989; Penland and Ghil, 1993].

[167] Penland [1996] provides an excellent review of vector-valued stochastic processes $\mathbf{X}(t)$ in continuous time and of their connection to discrete-time AR processes. $\mathrm{H}$ er review is well illustrated by the application of the basic concepts to the spectral analysis and AR prediction of sea surface temperatures in the tropical $\mathrm{Pa}$ cific. The details of the methodology are well beyond the scope of this brief review, but the basic idea is to use the data in order to estimate two matrices, $\mathbf{B}$ and $\mathbf{Q}$, in the model

$$
d \mathbf{X}=\mathbf{B} \mathbf{X} d t+d \xi(t) .
$$

Here $\mathbf{B}$ is the (constant) dynamics matrix and $\mathbf{Q}$ is the lag-zero covariance of the vector white-noise process $d \xi(t)$, considered here in continuous time.
[168] For the subtleties of time-continuous stochastic processes we refer to Penland [1996, and references therein]; $\xi(t)$ is a continuous, nowhere differentiable W iener process. For the present purposes, it suffices to recall that by the wide-sense stationarity assumption (see A ppendix A), B has to be stable, i.e., its eigenmodes have to decay in time. The latter property is the counterpart, in the present vector-matrix context, of the requirement that $a_{1}<0$ for the scalar AR(1) process of (1) and (16).

[169] The two matrices $\mathbf{B}$ and $\mathbf{Q}$ are related by a fluctuation-dissipation relation [Leith, 1975]:

$$
\mathbf{B C}(0)+\mathbf{C}(0) \mathbf{B}^{\mathrm{t}}+\mathbf{Q}=0,
$$

where $\mathbf{C}(\tau)=\mathscr{E}\left\{\mathbf{X}(t+\tau) \mathbf{X}^{\mathrm{t}}(t)\right\}$ is the lag-covariance matrix of the process $\mathbf{X}(t)$ being modeled and ()$^{\mathrm{t}}$ is the transpose of a vector or matrix. O ne proceeds to estimate the $\mathrm{G}$ reen's function $\mathbf{G}(\tau)=\exp (\mathbf{B} \tau)$ at a given lag $\tau_{0}$ from the sample of $\mathbf{C}(\tau)$ by

$$
\mathbf{G}\left(\tau_{0}\right)=\mathbf{C}\left(\tau_{0}\right) \mathbf{C}^{-1}(0) .
$$

Diagonalizing $\mathbf{G}\left(\tau_{0}\right)$ allows one to obtain $\mathbf{B}=\mathbf{B}_{\tau_{0}}$, via its eigenvalues and (left and right) eigenvectors.

[170] If (40) is the correct model for the process $\mathbf{X}(t)$, then the matrix $\mathbf{B}_{\tau}$ obtained for a certain $\tau=\tau_{0}$ should be, in fact, independent of $\tau$ [Penland and Ghil, 1993]. The ENM approach [Penland, 1989] differs from the original POP approach [Hasselmann, 1988] by (1) building on the theory of continuous-time stochastic processes and (2) considering the whole spectrum of $\mathbf{B}$, rather than preselected (pairs of) eigenvalues only.

[171] There are various reasons for which $\mathbf{G}(\tau)$, and hence $\mathbf{B}_{\tau}$, might be substantially different from one value of $\tau$ to another. These include problems and trade-offs in the choice of $\tau$, as well as nonlinearity, instability, or nonstationarity of the true process being sampled. The vector AR (1) method, whether in its POP or ENM form, works best when the spectrum is dominated by a single peak [see Von Storch et al., 1988; Penland, 1996, and references therein], which results in a well-separated pair of POPS or ENMS. The "tau test" of recomputing the eigendecomposition of $\mathbf{G}(\tau)$ and therewith of $\mathbf{B}_{\tau}$ for various $\tau$ values is particularly useful in determining the stability of the results, since error bars are, like for single-channel M EM and even more so, fairly difficult to come by.

[172] R ecently, Egger [1999] has extended the POP approach to higher-order vector AR processes. He calls such a vector $\mathrm{A} R(M)$ process a M OP model. M OPs can capture, in principle, a multiplicity of oscillating patterns that elude POPs. Still, they encounter difficulties when the underlying system that produces the signal is nonlinear, as is the case with POPs.

\subsection{Multichannel SSA (M-SSA)}

[173] M ultichannel SSA, often abbreviated as M-SSA, is a natural extension of SSA to a time series of vectors 
or maps, such as time-varying temperature or pressure distributions over the globe. The use of M -SSA for such multivariate time series was proposed theoretically, in the context of nonlinear dynamics, by Broomhead and King [1986b]. SSA can be applied, as explained in sections 1.2 and 2.1, in the context of systems of ODE s and the reconstruction of (the skeleton of) their attractor.

[174] M-SSA is a generalization of this approach to systems of partial differential equations and the study of the spatiotemporal structures that characterize the behavior of solutions on their attractor [Constantin et al., 1989; Temam, 1997]. It has been applied to intraseasonal variability of large-scale atmospheric fields by Kimoto et al. [1991], Keppenne and Ghil [1993], and Plaut and Vautard [1994], as well as to EN SO; for the latter, both observed data [Jiang et al., 1995a; Unal and Ghil, 1995] and coupled general circulation model (GCM) simulations [Robertson et al., 1995a, 1995b] were used.

[175] In the meteorological literature, extended EOF (EEOF) analysis is often assumed to be synonymous with M-SSA [Von Storch and Zwiers, 1999]. The two methods are both extensions of classical principal component analysis (PCA) [Preisendorfer, 1988] but they differ in emphasis: EE OF analysis [Barnett and Hasselmann, 1979; Weare and Nasstrom, 1982; Lau and Chan, 1985] typically utilizes a number $L$ of spatial channels much greater than the number $M$ of temporal lags, thus limiting the temporal and spectral information. In M SSA, on the other hand, on the basis of the singlechannel experience reviewed in sections 2 and 3.3, one usually chooses $L \leq M$ (see also A ppendix A). Often M-SSA is applied to a few leading PCA components of the spatial data, with $M$ chosen large enough to extract detailed temporal and spectral information from the multivariate time series.

[176] Let $\left\{X_{l}(t): l=1, \ldots, L ; t=1, \ldots, N\right\}$ be an $L$-channel time series with $N$ data points given at equally spaced intervals $n \Delta t$. We assume that each channel $l$ of the vector $\mathbf{X}(n \Delta t)$ is centered and stationary in the weak sense.

[177] The generalization of SSA to a multivariate time series requires the construction of a "grand" block matrix $\widetilde{\mathbf{T}}_{\mathbf{X}}$ for the covariances that has the form

$\tilde{\mathbf{T}}_{\mathbf{X}}=\left(\begin{array}{cccccc}\mathbf{T}_{1,1} & \mathbf{T}_{1,2} & \cdot & \cdot & \cdot & \mathbf{T}_{1, L} \\ \mathbf{T}_{2,1} & \mathbf{T}_{2,2} & \cdot & & & \cdot \\ \cdot & \cdot & \cdot & \cdot & & \cdot \\ \cdot & \cdot & \cdot & \cdot & \mathbf{T}_{l, l^{\prime}} & \cdot \\ \cdot & \cdot & \cdot & \cdot & & \cdot \\ \cdot & & & \cdot & \cdot & \mathbf{T}_{L-1, L} \\ \mathbf{T}_{L, 1} & \cdot & \cdot & \cdot & \mathbf{T}_{L, L-1} & \mathbf{T}_{L, L}\end{array}\right)$.

E ach block $\mathbf{T}_{l, l^{\prime}}$ is a matrix that contains estimates of the lag covariance between channels $l$ and $l^{\prime}$. Extending the approach of Vautard and Ghil [1989] (see section 2.2), Plaut and Vautard [1994] proposed to obtain a "least biased" estimator by using, for each lag $m(m=0, \ldots$, $M-1$ ), the longest-possible segment of each channel.
Thus the entries $\left(j, j^{\prime}\right)$ of each block $\mathbf{T}_{l, l^{\prime}}$ can be written as

$\left(T_{l, l^{\prime}}\right)_{j, j^{\prime}}=\frac{1}{\tilde{N}} \sum_{n=\max \left(1,1+j-j^{\prime}\right)}^{\min \left(N, N+j-j^{\prime}\right)} X_{l}(t) X_{l^{\prime}}\left(t+j-j^{\prime}\right)$,

where $\tilde{N}$ is a factor that depends on the range of summation, to wit,

$\tilde{N}=\min \left(N, N+j-j^{\prime}\right)-\max \left(1,1+j-j^{\prime}\right)+1$

(compare equation (6)).

[178] N ote that unlike in the single-channel case, here $\mathbf{T}_{l, l^{\prime}}$ is Toeplitz but not symmetric. Its main diagonal contains the Vautard and Ghil [1989] estimate of the lag-zero covariance of channels $l$ and $l^{\prime}$. The diagonals in the lower left triangle of $\mathbf{T}_{l, l^{\prime}}$ contain the lag- $m$ covariance of channels $l$ and $l^{\prime}$, with $l^{\prime}$ leading $l$, while the diagonals in the upper right triangle contain the covariances with $l$ leading $l^{\prime}$. E quation (44) ensures that $\mathbf{T}_{l^{\prime}, l}=$ $\left(\mathbf{T}_{l, l^{\prime}}\right)^{\mathrm{t}}$ so that $\tilde{\mathbf{T}}_{\mathbf{X}}$ is symmetric, but it is not Toeplitz. Note that the original formula of Plaut and Vautard [1994, equation (2.5)] does not, in fact, yield a symmetric matrix. M ore generally, we only expect to obtain a grand matrix $\widetilde{\mathbf{T}}_{\mathbf{X}}$ that is both symmetric and of Toeplitz form provided the spatial field being analyzed is statistically homogeneous; that is, provided its statistics are invariant with respect to arbitrary translations and rotations.

[179] A n alternative approach [Broomhead and King, 1986a, 1986b; Allen and Robertson, 1996] to computing the lagged cross covariances is to form the multichannel trajectory matrix $\tilde{\mathbf{X}}$ by first augmenting each channel $\left\{X_{l}(t): t=1, \ldots, N\right\}, 1 \leq l \leq L$, of $\mathbf{X}$ with $M$ lagged copies of itself,

$$
\tilde{\mathbf{X}}_{l}=\left(\begin{array}{cccc}
X_{l}(1) & X_{l}(2) & \ldots & X_{l}(M) \\
X_{l}(2) & X_{l}(3) & \ldots & X_{l}(M+1) \\
\cdot & \cdot & \ldots & \cdot \\
X_{l}\left(N^{\prime}-1\right) & \cdot & . & X_{l}(N-1) \\
X_{l}\left(N^{\prime}\right) & X_{l}\left(N^{\prime}+1\right) & \cdots & X_{l}(N)
\end{array}\right), \quad \text { (46) }
$$

and then forming the full augmented trajectory matrix:

$$
\tilde{\mathbf{D}}=\left(\tilde{\mathbf{X}}_{1}, \tilde{\mathbf{X}}_{2}, \ldots, \tilde{\mathbf{X}}_{L}\right) \text {. }
$$

[180] These two steps generalize (5) and (9). The grand lag-covariance matrix $\tilde{\mathbf{C}}_{\mathbf{X}}$ that is analogous to $\mathbf{C}_{X}$ in (9) is given by

$\tilde{\mathbf{C}}_{\mathbf{X}}=\frac{1}{N^{\prime}} \tilde{\mathbf{X}} \tilde{\mathbf{X}}^{\mathrm{X}}=\left(\begin{array}{cccccc}\mathbf{C}_{1,1} & \mathbf{C}_{1,2} & \cdot & \cdot & \cdot & \mathbf{C}_{1, L} \\ \cdot & \mathbf{C}_{2,2} & \cdot & \cdot & \cdot & \cdot \\ \cdot & & \cdot & \cdot & \cdot & \cdot \\ \cdot & & \cdot & \cdot & \mathbf{C}_{l, l^{\prime}} & \cdot \\ \cdot & & \cdot & \cdot & \cdot & \cdot \\ \mathbf{C}_{L, 1} & \mathbf{C}_{L, 2} & \cdot & \cdot & \cdot & \mathbf{C}_{L, L}\end{array}\right)$,

where $N^{\prime}$ is given in Table 1. The blocks of $\tilde{\mathbf{C}}_{\mathbf{X}}$ are given by 


$$
\mathbf{C}_{l, l^{\prime}}=\frac{1}{N^{\prime}} \tilde{\mathbf{X}}_{l}^{\mathrm{t}} \tilde{\mathbf{X}}_{l^{\prime}}
$$

with entries

$\left(C_{l, l^{\prime}}\right)_{j, j^{\prime}}=\tilde{C}_{I J}=\frac{1}{N^{\prime}} \sum_{t=1}^{N^{\prime}} X_{l}(t+j-1) X_{l^{\prime}}\left(t+j^{\prime}-1\right)$,

where $I=j+M(l-1)$ and $J=j^{\prime}+M\left(l^{\prime}-1\right)[$ Allen and Robertson, 1996]. Here $\mathbf{C}_{l, l^{\prime}}$ is neither Toeplitz nor symmetric, but again $\mathbf{C}_{l^{\prime}, l}=\left(\mathbf{C}_{l, l^{\prime}}\right)^{\mathrm{t}}$ and hence $\tilde{\mathbf{C}}_{\mathbf{X}}$ is symmetric.

[181] Each block $\mathbf{C}_{l^{\prime}, l}$ contains, like $\mathbf{T}_{l, l^{\prime}}$ in (43), an estimate of the lag covariances of the two channels $l$ and $l^{\prime}$. The difference between the Toeplitz method of $\mathrm{Vau}$ tard and Ghil [1989] and the trajectory matrix method of Broomhead and King [1986a, 1986b] in estimating the lag-covariance matrix, when applied to M-SSA, consists of calculating (43) and (44) versus (48)-(50). The Toeplitz method extracts, according to the fixed lag $m$ that is being considered at the moment, the two longest matching segments from the two channels $l$ and $l^{\prime}$; the matching is determined by the requirement that for each entry in the designated trailing channel, there exist an entry in the designated leading channel that is $m$ time steps "ahead." It then uses the same estimated lag covariance for all entries along the appropriate diagonal in the block and thus yields a Toeplitz-form submatrix.

[182] The trajectory matrix method gradually slides, regardless of $m$, the same $N^{\prime}$-long windows over the two channels. To compute the first entry in the diagonal that contains the lag- $m$ covariances, the two matching windows are situated so that one starts at the first time point of the trailing channel and at the $(m+1)$ st point of the leading channel. B oth windows are then slid forward by one point in time to produce the second entry. This results in slightly different values, from entry to entry, until the last point of the leading channel is covered and the $(M-m)$ th entry of the diagonal, which is the last one, is calculated. This latter method thus retains detailed information on the variation of the lag-covariance estimates from one pair of segments of the channels $l$ and $l^{\prime}$ to another, while the Toeplitz method produces a single, and smoother, global estimate for each lag $m$.

[183] Diagonalizing the $L M \times L M$ matrix $\tilde{\mathbf{C}}_{\mathbf{X}}$ or $\tilde{\mathbf{T}}_{\mathbf{X}}$ yields $L M$ eigenvectors $\left\{\mathbf{E}^{k}: 1 \leq k \leq L M\right\}$ that are not necessarily distinct. The extent to which the eigenpairs $\left(\lambda_{k}, \mathbf{E}^{k}\right)$ obtained by diagonalizing $\tilde{\mathbf{C}}_{\mathbf{X}}$ equal those obtained from $\widetilde{\mathbf{T}}_{\mathbf{X}}$ is a good indication of the robustness of the M-SSA results. E ach eigenvector $\mathbf{E}^{k}$ is composed of $L$ consecutive $M$-long segments, with its elements denoted by $E_{l}^{k}(j)$. The associated space-time PCs $\mathbf{A}^{k}$ are single-channel time series that are computed by projecting $\tilde{\mathbf{X}}$ onto the EOFs:

$$
A^{k}(t)=\sum_{j=1}^{M} \sum_{l=1}^{L} X_{l}(t+j-1) E_{l}^{k}(j),
$$

\section{Niño-3 SSTA and RCs}

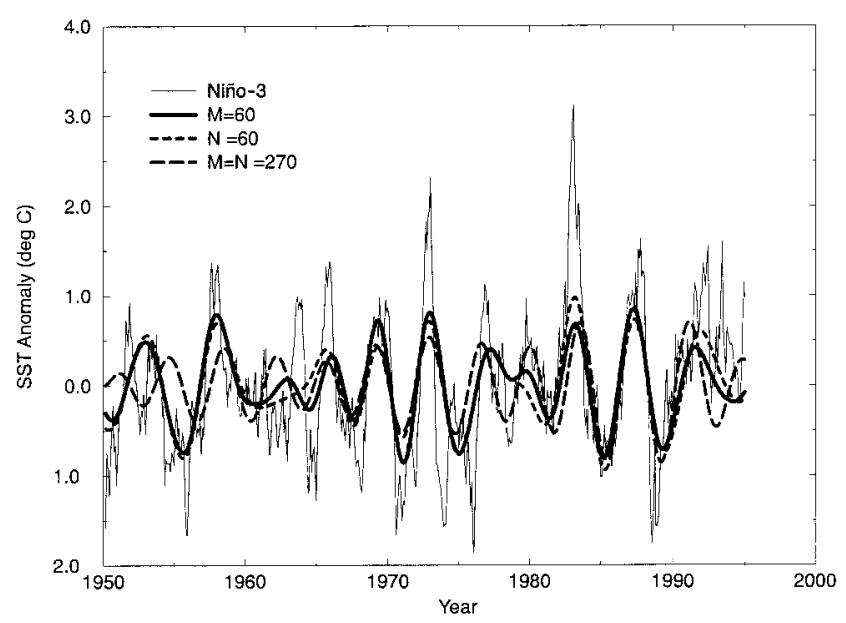

Figure 18. SST anomalies averaged over the Niño-3 region. Light solid line indicates Global Sea Ice and Sea Surface Temperature (GISST) data; bold curves are R Cs 2-3 for $M=$ 60 (solid) and $N^{\prime}=60$ (short dashed), as well as R C s 3- 4 for $M=N^{\prime}=270$ (long dashed).

where $t$ varies from 1 to $N^{\prime}$. This is the multichannel counterpart of (10).

[184] F or a given set of indices $\mathscr{K}, \mathrm{R}$ C s are obtained by convolving the corresponding PCS with the EOFs. Thus the $k$ th $\mathrm{RC}$ at time $t$ for channel $l$ is given by

$$
R_{l}^{k}(t)=\frac{1}{M_{t}} \sum_{j=L_{t}}^{U_{t}} A^{k}(t-j+1) E_{l}^{k}(j) .
$$

A $s$ in (11), the normalization factor $M_{t}$ equals $M$, except near the ends of the time series, and the sum of all the $\mathrm{R}$ Cs recovers the original time series [Plaut and Vautard, 1994], as it does in the single-channel case. The factor $M_{t}$, as well as the lower and upper bounds of summation, $L_{t}$ and $U_{t}$, are given by the same equation, equation (12), as in the single-channel case.

[185] As an example, we apply M -SSA to the nearglobal data set of monthly sea surface temperatures from the Global Sea Ice and Sea Surface Temperature (GISST) data set [Rayner et al., 1995] for 1950-1994, from $30^{\circ} \mathrm{S}$ to $60^{\circ} \mathrm{N}$, on a $4^{\circ}$-latitude by $5^{\circ}$-longitude grid. The mean seasonal cycle was subtracted at the outset, and the data were prefiltered with standard PCA [Preisendorfer, 1988] to retain the 10 leading spatial PCs that describe $55.2 \%$ of the variance. This favors the association of larger decorrelation times with larger spatial scales, as expected for climatic [Fraedrich and Boettger, 1978] and other geophysical fields, and the channels are uncorrelated at zero lag. We use therewith $L=10$ channels and $N=540$ months; no other filtering or detrending was applied. The SST anomalies of this data set were averaged over the $\mathrm{Niño-3}$ area in the eastern tropical Pacific $\left(5^{\circ} \mathrm{S}-5^{\circ} \mathrm{N}, 90^{\circ}-150^{\circ} \mathrm{W}\right)$, and the evolution of these averaged anomalies is shown in Figure 18 (light 
a) phase 1

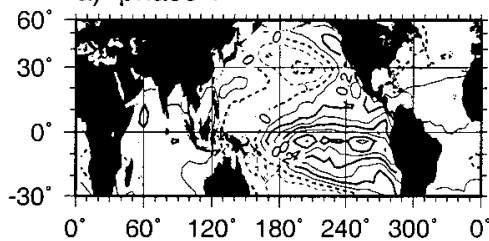

b) phase 2

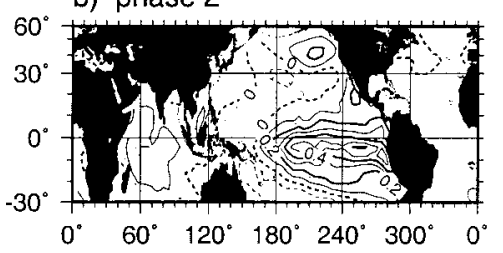

c) phase 3

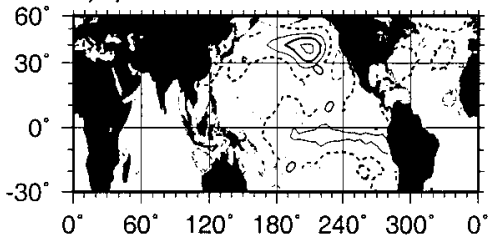

d) phase 4

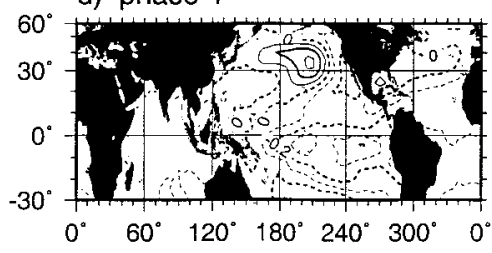

e) phase 5

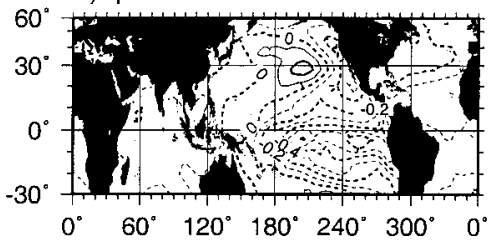

f) phase 6

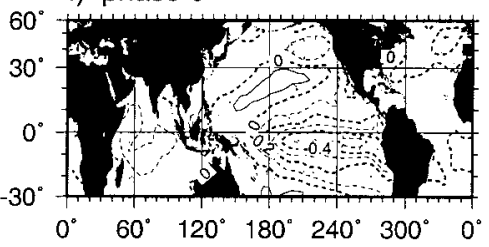

g) phase 7

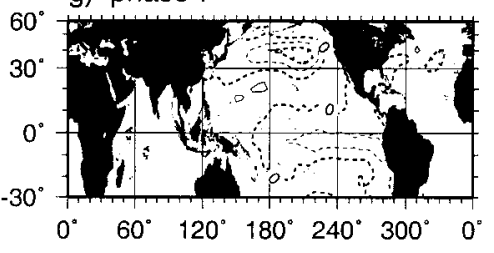

h) phase 8

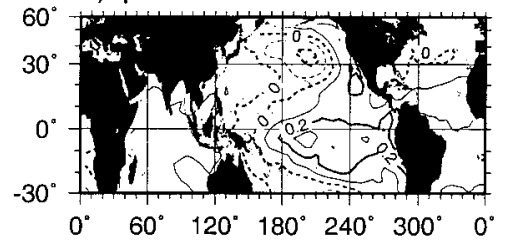

Figure 19. Phase composites for the low-frequency EI N iño-Southern O scillation (E NSO) cycle, as given by R Cs 2-3 with $N^{\prime}=60$. The cycle is divided into eight segments of $45^{\circ}$ each, using the first spatial PC as an index. Contour interval is $0.1^{\circ} \mathrm{C}$.

solid curve).

[186] F or comparison with previous EN SO studies we choose $M=60$ months, i.e., a 5-year $M$ window. To demonstrate the usage of $N^{\prime}$ windows versus $M$ windows (see A ppendix A, especially equation (A 1 ) and Figure A 1, for the definition of "complementary windows"), we also set $M=480$, which yields an $N^{\prime}$ window of 5 years, and $M=270$, which yields the $M$ and the $N^{\prime}$ windows both equal to $N / 2$.

[187] We use here the lag-covariance matrices $\tilde{\mathbf{C}}_{\mathbf{X}}$ that are based on the trajectory matrix approach of Broomhead and King [1986a, 1986b] for ease of comparison. In all three cases, $M L>N^{\prime}$, so that it is more efficient to diagonalize the reduced $\left(N^{\prime} \times N^{\prime}\right)$ covariance matrix with elements given by $(\mathrm{A} 1)$, rather than the $(M L \times$ $M L$ ) matrix whose elements are given by (48).

[188] The leading oscillatory pair over the entire domain has a quasi-quadrennial period for all three values of $M$, as illustrated by the spatial average of the respective RCs over the Niño-3 region (Figure 18). The two reconstructions for $M=60$ (bold solid curve) and $N^{\prime}=$
60 (short-dashed curve) isolate a very similar frequencymodulated mode, with a slightly longer period before 1965 (see Figure 8 and discussion there). The quasi-quadrennial pair (modes 2 and 3 in these two cases), however, only accounts for $20.0 \%$ variance with $M=60$ against $30.8 \%$ for $N^{\prime}=60$. This difference arises because the reduced covariance matrix has rank 60 in the latter case, which maximizes data compression, compared with rank $M L=$ 600 for $M=60$. The smaller one of $M$ and $N^{\prime}$ determines the approximate spectral resolution $1 / N^{\prime}$ or $1 / M$. Choosing $M=N^{\prime}=270$ yields the maximum spectral resolution but captures less variance for the oscillatory pair of interest: only $12.1 \%$ variance for the quasi-quadriennial pair, formed in this case by modes 3 and 4 .

[189] Figure 19 shows the spatiotemporal evolution of the quasi-quadrennial mode ( RCs 2-3) for $N^{\prime}=60$, using the phase-compositing procedure of Plaut and Vautard [1994]. The maps (not shown) are almost indistinguishable for $M=60$ (same pair) and $M=N^{\prime}=270$ (R Cs 3-4). The succession of panels in Figure 19 illustrates the progression of the oscillation, keyed to the 
phase index constructed by using the leading spatial PC of R Cs 2-3 [Moron et al., 1998].

[190] The maps are dominated by the classic EI Niño signature over the tropical Pacific, together with the well-known "horseshoe" pattern over the extratropical Pacific [see Chao et al., 2000, and references therein]. A mplitudes elsewhere are weak: In the northwestern Indian Ocean, though, there is some suggestion of a warming during EI Niño and cooling during La Niña events that lags the eastern equatorial Pacific by $1 / 8$ cycle ( $\sim 6$ months). $O$ ver the tropical $A$ tlantic, there is also a small warming concurrent with the peak phase of EI Niño and cooling simultaneously with La N iña.

[191] A s in the single-channel case, a test of statistical significance is needed to avoid spurious oscillations that are spatially smooth looking and might arise from $\mathrm{M}$ SSA of finitely sampled noise processes. The complementary $N^{\prime}$-window approach of (A 1) (see A ppendix A) allows the univariate SSA Monte Carlo test of Allen [1992; see also Allen and Smith, 1996] to be extended to $\mathrm{M}$-SSA in a straightforward manner, provided $M L>$ $N^{\prime}$, so that the reduced covariance matrix is completely determined and has full rank. A s shown in our example above, $N^{\prime}$ can always be chosen sufficiently small, so that the complementary window $N^{\prime}$ used in (A 1) determines the spectral resolution.

[192] Details of this essentially univariate test are given by Allen and Robertson [1996]. The usefulness of the test depends in an essential way on the channels being uncorrelated at zero lag or very nearly so. In the example at hand, the decorrelation condition holds exactly, since we use the PCS of spatial EOF analysis. When using time series from grid points that are sufficiently far from each other for decorrelation to be near perfect, the test can still be useful.

[193] In this test the data series together with a large ensemble of red-noise surrogates are projected onto the eigenmodes of the reduced covariance matrix of either the data or the noise. The statistical significance of the projections is estimated as in the single-channel test described in section 2.3. The noise surrogates are constructed to consist of univariate AR (1) segments, one per channel, that match the data in autocovariance at lag 0 and $\operatorname{lag} 1$, channel by channel. The reason an essentially univariate test can be applied is because the eigenmodes do not depend on cross-channel lag covariance, provided $N^{\prime}$ is interpreted as the spectral window used in (A 1). Since the test is biased if we project onto the data eigenmodes, we project onto the eigenmodes provided by the covariance matrix of the AR (1) noise.

[194] Figure 20 shows the result of the test applied for $M=N^{\prime}=270$, the maximum effective resolution. In Figure 20 the projections are plotted against the dominant frequencies associated with each noise eigenvector. Since the latter are near sinusoidal in this case, the resulting spectrum is closely related to a traditional Fourier power spectrum. Both the quasi-quadrennial and the quasi-biennial modes pass the test at the $95 \%$

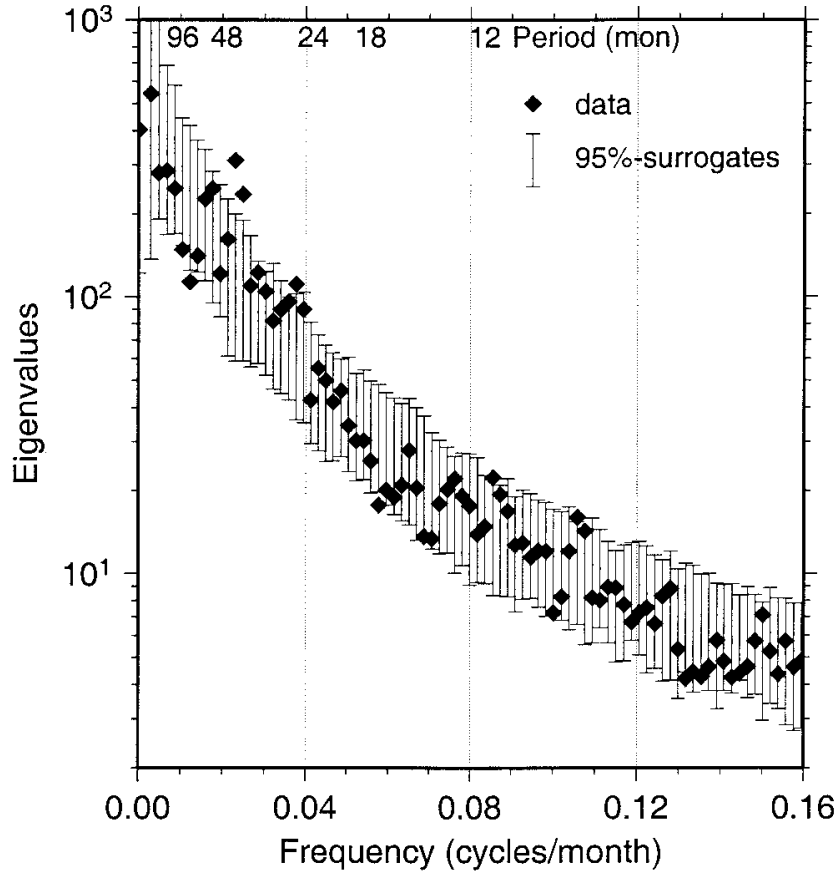

Figure 20. M onte Carlo significance test for interannual oscillations in the GISST data. The first 10 spatial PCs are projected onto AR (1) basis vectors with $M=270$. E rror bars show the $95 \%$ confidence interval constructed from an AR (1) surrogate data ensemble of 1000. In comparing with Figure 7, note that the distribution of the points along the abscissa here is uniform, since the attribution of the dominant frequency for A R (1) basis vectors is simple [Vautard and Ghil, 1989; Allen, 1992].

level. They are well separated in frequency by about $1 /(20$ months), which far exceeds the spectral resolution of $1 / M=1 / N^{\prime}=1 /(270$ months $) \approx 1 /(22$ years $)$. The two modes are thus significantly distinct from each other spectrally, in agreement with the univariate SOI results of Figures 12 and 14, using MEM and MTM, respectively.

\section{SUMMARY AND PERSPECTIVES}

\subsection{Summary}

[195] We have reviewed a number of recent developments in the spectral analysis of climatic and other geophysical time series. Basic ideas and their connections to dynamical systems theory were emphasized. The methods arising from these ideas and their numerical implementation were outlined and illustrated.

[196] The key features of the methods discussed in this review are summarized in Table 3 (see also Table 1 of Ghil and Taricco [1997]). We have tried to provide up-to-date information on the most refined and robust statistical significance tests available for each one of the three methods that were discussed in depth: SSA, MEM , and M TM . Still, this group of authors feels that none of these methods by itself can provide entirely reliable 
TABLE 3. Comparison Table of Spectral Methods

\begin{tabular}{|c|c|c|c|c|c|c|}
\hline Method & Parametric & Lines & $\begin{array}{l}\text { Continuous } \\
\text { Spectrum }\end{array}$ & Modulation & Error Bars & Remarks \\
\hline BT (section 3.2) & no & low resolution & $\begin{array}{l}\text { variance } \\
\text { reduction }\end{array}$ & partially ${ }^{a}$ & $\begin{array}{l}\text { confidence } \\
\text { intervals }^{b}\end{array}$ & simple \\
\hline MEM /AR (section 3.3) & yes & $\begin{array}{l}\text { high } A R \\
\text { order }\end{array}$ & $\begin{array}{l}\text { low AR } \\
\text { order }\end{array}$ & yes $^{d}$ & $\begin{array}{l}\text { confidence } \\
\text { intervals }\end{array}$ & SSA prefiltering helps \\
\hline $\begin{array}{l}\text { POP and ENM (section } \\
4.1 \text { ) }\end{array}$ & yes & $\begin{array}{l}\text { in space and } \\
\text { time }\end{array}$ & no & no & $\begin{array}{l}\text { robustness via } \\
\tau \text { test }\end{array}$ & $\begin{array}{l}\text { inverse-model dynamics: } \\
\text { linear and stable }\end{array}$ \\
\hline MTM (section 3.4) & no & $\begin{array}{l}\text { harmonic } \\
\text { analysis }\end{array}$ & $\begin{array}{l}\text { variance } \\
\text { reduction }\end{array}$ & $\begin{array}{l}\text { amplitude and } \\
\text { phase; } \\
\text { frequency via } \\
\text { ESA }\end{array}$ & $\begin{array}{l}F \text { test }^{g} ; \\
\text { confidence } \\
\text { intervals }\end{array}$ & $\begin{array}{l}\text { cost of DPSS } \\
\text { computation }\end{array}$ \\
\hline SSA (sections 2 and 4.2) & no & low resolution & yes $^{i}$ & $\begin{array}{l}\text { amplitude and } \\
\text { phase; some } \\
\text { frequency shifts }\end{array}$ & $\begin{array}{l}\text { heuristic error } \\
\text { bars; M onte } \\
\text { Carlo } \\
\text { confidence } \\
\text { intervals }^{\mathrm{h}}\end{array}$ & $\begin{array}{l}\text { detects nonlinear } \\
\text { oscillations }\end{array}$ \\
\hline W avelets (section 2.4 ) & no & $\begin{array}{l}\text { variable } \\
\quad \text { resolution }\end{array}$ & $\cdots$ & $\begin{array}{l}\text { amplitude and } \\
\text { phase and } \\
\text { frequency }\end{array}$ & $\cdots$ & $\begin{array}{l}\text { nonstationary signals, } \\
\text { local in time and } \\
\text { frequency }\end{array}$ \\
\hline
\end{tabular}

\footnotetext{
${ }^{a} O$ nly one degree of freedom in envelope, via complex demodulation.

${ }^{b}$ Confidence intervals are obtained with respect to $A R(0)$ and $A R(1)$ null hypotheses.

${ }^{\mathrm{C}} \mathrm{H}$ igh $\mathrm{AR}$ orders can produce very good resolution but spurious peaks too.

dU nderlying AR model can describe modulated oscillation.

ePOP is used to detect spatiotemporal patterns on an AR (1) background.

${ }^{f}$ E volutive spectral analysis.

${ }^{\mathrm{g} F}$ test for harmonic analysis has a high resolution but produces spurious peaks when the spectrum is continuous.

${ }^{\mathrm{h}}$ Confidence intervals are obtained from the null hypothesis of $A R(0)$ or $A R(1)$ noise.

iA pproximated by the near-constant slopes in the spectral "scree diagram."

iThe choice of the wavelet basis is delicate and involves a trade-off between time resolution and scale resolution.
}

results, since every statistical test is based on certain probabilistic assumptions about the nature of the physical process that generates the time series of interest. Such mathematical assumptions are rarely, if ever, met in practice.

[197] To establish higher and higher confidence in a spectral result, such as the existence of an oscillatory mode, a number of steps can be taken. First, the mode's manifestation is verified for a given data set by the best battery of tests available for a particular spectral method. Second, additional methods are brought to bear, along with their significance tests, on the given time series. This process was illustrated by the SOI time series in the present review. Vautard et al. [1992] and Yiou et al. [1996] provided similar illustrations for a number of synthetic time series, as well as for the Intergovernmental Panel on Climate Change (IPCC) time series of global surface-air temperatures [IPCC, 1990] and for the proxy record of deuterium isotopic ratio [Jouzel et al., 1993] that reflects local air temperatures at V ostok station in the A ntarctica.

[198] The application of the different univariate methods described here and of their respective batteries of significance tests to a given time series is facilitated by the SSA-M TM Toolkit, which was originally developed by Dettinger et al. [1995a]. The Toolkit has evolved as freeware over the last 7 years to become more effective, reliable, and versatile. Its latest version is available at http://www.atmos.ucla.edu/tcd/ssa.
[199] The third step on the road to genuine confidence in a given oscillatory mode is to obtain additional time series that might exhibit the mode of interest, if indeed it is present. For ENSO, this includes, besides SOI, the $\mathrm{N}$ iño-3 sea surface temperatures shown in Figure 18, as well as the additional data sets discussed in connection with Figure 6 in section 2.2 here (see also Rasmusson et al. [1990], Keppenne and Ghil [1992], Jiang et al. [1995a], and Unal and Ghil [1995] for instrumental data and Mann and Park [1999, and references therein] for proxy records). The existence of two oscillatory climate modes with interannual periodicities, one quasi-biennial, the other with a period of 4- 6 years, while not present in every record and for every method, has been established with great confidence. These two modes are thus definitely part of the skeleton of the attractor for climate dynamics on interannual timescales.

[200] The fourth and most difficult step on the road of confidence building is that of providing a convincing physical explanation of an oscillation, once we have full statistical confirmation for its existence. This step consists of building and validating a hierarchy of models for the oscillation of interest [see Ghil, 1994; Ghil and Robertson, 2000]. The modeling step is distinct from and thus fairly independent of the statistical analysis steps discussed up to this point. It can be carried out before, after, or in parallel with the other three steps.

[201] M odel validation requires specifically the theoretical prediction of an oscillatory feature not yet ob- 
tained by time series analysis. The prediction might involve a spatial feature not yet diagnosed from existing observations or as-yet-unobserved spectral features: harmonics, subharmonics, or combination tones. The subsequent detection in new data sets of such a theoretically predicted spatial or spectral feature, while never definitive, tends to greatly bolster the case for the proposed model. It thus reinforces more than anything else the confidence in the existence and importance of the mode under discussion.

[202] We turn therewith to the implications that the discovery by spectral methods of oscillatory behavior can have for the deeper understanding of climatic variability and for its real-time prediction.

\subsection{Implications for Understanding}

[203] We have introduced in section 1 the concept of a ghost limit cycle ( $F$ igure 1 ) as the underpinning for the dynamic usefulness of a spectral peak. In a number of instances, from intraseasonal oscillations [Ghil et al., 1991] through paleoclimatic variability [Ghil, 1994], the robust identification of an oscillatory mode in observations or proxy records has gone hand in hand with the description of a $\mathrm{H}$ opf bifurcation in a single model or a full hierarchy of models [Ghil and Robertson, 2000]. Such a bifurcation gives rise, via an oscillatory instability and its nonlinear saturation, to a stable limit cycle. This limit cycle is destabilized in turn, as the forcing on the system increases or the constraints on it (such as viscous or eddy dissipation) decrease; it thus becomes a "ghost limit cycle." The process of successive bifurcations engenders additional ghost limit cycles and thus leads to multiply periodic, and eventually irregular, behavior [Guckenheimer and Holmes, 1983; Ghil and Childress, 1987; Schuster, 1988].

[204] In the present paper we used seasonal-to-interannual climate variability as the A riadne's thread to help guide the interested reader through the labyrinth of methodological exposition. As mentioned in sections $2.2,3.3$, and 4.2 , the data-adaptive $\mathrm{S} / \mathrm{N}$ enhancement obtained by applying SSA to univariate and multivariate climatic time series covering this variability led to the robust identification of separate 4/1- and 4/2-year peaks. A $n$ additional, 4/3-year peak [Robertson et al., 1995b; Ghil and Robertson, 2000] completes a set of three subharmonics of the seasonal cycle that characterize the Devil's staircase mechanism of interaction between the ENSO's intrinsic, self-sustained oscillation in the coupled ocean-atmosphere system and the seasonal forcing. This mechanism involves partial phase locking between the two oscillations and has been studied in detail in simple and intermediate ENSO models [Chang et al., 1994; Jin et al., 1994; Tziperman et al., 1994; Saunders and Ghil, 2001], in which the period of the intrinsic E NSO cycle varies between 2 and 3 years, depending on models and parameters.

[205] The separation of two or three peaks (quasiquadrennial, quasi-biennial, and sometimes but not al- ways, 16-17 months) and the spatiotemporal variability associated with them have been ascertained in various data sets [Rasmusson et al., 1990; Jiang et al., 1995a], as well as in coupled general circulation models [Robertson et al., 1995a, 1995b; Ghil and Robertson, 2000]. The D evil's staircase thus provides the most plausible explanation so far for the warm events peaking typically in boreal winter, as well as for the irregularity of the EI $N$ iños' spacing from one warm event to the next (see also discussion at the end of section 2.4 and further below).

[206] A similar dialog between spectral studies of interdecadal variability and dynamical studies of oscillatory model behavior is taking shape. Interdecadal modes of variability at 10-11, 14-15, and 25-27 years were initially identified, by combining the application of various spectral methods described in sections 2 and 3, to wit, SSA, MEM, and MTM, in both global [Ghil and Vautard, 1991; Vautard et al., 1992] and regional [Plaut et al., 1995] time series. Allen and Smith [1994, 1996] and Mann and Lees [1996] examined further the level of significance of those spectral features against a red-noise background. Allen and Smith [1994] described the spatial patterns associated with the variability at 10-11 and 25-27 years by using a method related to M-SSA .

[207] The spectral peak at 14-15 years was confirmed by SSA of coral reef proxy records from the tropical South Pacific by Quinn et al. [1993]. Mann and Park [1994] described this mode's spatial pattern on a global scale by using MTM-related multichannel methods applied to instrumental temperature data [see also Mann and Park, 1999]. V ariability in the South A tlantic basin with a near periodicity of 14-16 years has been described by Venegas et al. [1997] and Robertson and Mechoso [2000].

[208] On the other hand, a plethora of oscillatory mechanisms is emerging in two-dimensional [Quon and Ghil, 1995], as well as in fully three-dimensional [Weaver et al., 1993; Chen and Ghil, 1995; Rahmstorf, 1995], models of the ocean's thermohaline circulation (see summary table, with further references, given by Ghil [1994]). Coupled ocean-atmosphere models are also producing decadal and interdecadal oscillations that seem to resemble the purely oceanic ones [Delworth et al., 1993; Chen and Ghil, 1996] or differ from them in substantial ways [Latif and Barnett, 1994; Robertson, 1996].

[209] As in the case of ENSO variability, a full hierarchy of models will probably be needed to clarify the connections between the (dimly) observed and the (imperfectly) modeled oscillations on the decadal and interdecadal timescales. A particularly interesting set of questions deals with the 14-15 year oscillation and its pattern and physical causes. The details of its spatiotemporal evolution in the North A tlantic have been described by Moron et al. [1998], and their implications for prediction have been outlined by Sutton and Allen [1997]. These details seem to point to the intrinsic variability of the N orth A tlantic's wind-driven circulation as 
a likely source [Jiang et al., 1995c; Speich et al., 1995; Dijkstra and Molemaker, 1999]. Chao et al. [2000], on the other hand, have described a 15-20 year oscillation encompassing the entire Pacific basin's sea surface temperatures and roughly symmetric about the equator. Its approximate subharmonic, with a period of 31-35 years, appears in coral records of both $\delta^{18} \mathrm{O}$ and $\delta^{13} \mathrm{C}$ [Quinn et al., 1993; Boiseau et al., 1999], as well as in 2300 years of tree ring records from Tasmania [Cook et al., 1995].

[210] A re the three oscillations, in the N orth A tlantic, South A tlantic, and Pacific, phase-locked, as suggested by Mann and Park [1994]? If so, do they have a common origin that is purely oceanic, or is it due to coupled ocean-atmosphere processes? If not, what are their separate origins?

[211] A $n$ important aspect of nonlinear oscillations in a complex system with multiple timescales, like the climate system, is their modulation in amplitude, phase, and frequency (see Table 3). We showed that SSA can follow well, via reconstructed components (R CS), variations in signal amplitude and phase that are associated with a fairly broad spectral peak [Ghil and Mo, 1991a; Plaut and Vautard, 1994; Dettinger and Ghil, 1998]. M TM -based harmonic analysis can be adapted, by using a sliding window, to follow the slow shift in time of a sharp line's frequency [Yiou et al., 1991; Birchfield and Ghil, 1993]. Wavelet analysis provides an even more natural way of following quasi-adiabatic, gradual changes in the natural frequency of a climatic oscillator [Meyers et al., 1993; Yiou et al., 1995]. Suitable significance criteria for wavelet analysis results [Flandrin, 1999] need to be adapted further to the problems of following slow changes in frequency, as well as in amplitude and phase, of climate oscillations.

[212] We showed that multiscale SSA, which can be viewed as a data-adaptive form of wavelet analysis, is a novel and useful tool for the reliable identification of sharp, relatively sudden changes in frequency [Yiou et al., 2000]. Its results, when applied to SOI, support further the D evil's staircase theory of ENSO, by highlighting the sudden jump in the low-frequency oscillation's periodicity in the early 1960s (see also the discussion of secular warming in the tropical Pacific and its effects on the evolution of ENSO variability over the twentieth century by Park and Mann [2000]).

\subsection{Implications for Prediction}

[213] In the previous section we discussed the need for validating physical models of a spectrally detected oscillatory mode by formulating and verifying theoretical predictions of the oscillation's features, spatial or temporal, that have not yet been observed. There is, in climate dynamics and other geosciences, another type of prediction that can greatly help increase confidence in the description and explanation of the various time series generated by a given process, namely, the forecasting of the process's future evolution in time. In fact, this is also one of the main purposes of time series analysis [Wiener, 1949; Box and Jenkins, 1970; Priestley, 1981b; Ghil, 1997].

[214] The combination of SSA with M EM provides an immediate application of the spectral methods discussed herein to prediction. The key idea, illustrated in section 3.3, is that each significant RC obtained from an SSA analysis is a narrowband time series. It can therefore be predicted fairly robustly by computing a low-order $\mathrm{A} \mathrm{R}(M)$ process that fits this $\mathrm{R} \mathrm{C}$ over the time interval available, up to the present epoch. The separate $R C$ predictions can then be combined to form a partial reconstruction of the entire time series' future evolution [Keppenne and Ghil, 1992; Vautard et al., 1992].

[215] The decision about which RCs to use for this SSA-MEM prediction is based on a trade-off between the amount of variance one hopes to predict and the reliability of the prediction. The former consideration suggests using all significant components (see discussion in sections 2.2 and 2.3), the latter suggests restricting oneself to the oscillatory ones [Plaut et al., 1995; Ghil and Jiang, 1998]. The accuracy of the prediction depends, in either case, on the extent to which the available data from the past do determine the regular, i.e., periodic and multiply periodic, behavior of the time series. Instrumental temperature data over the last few centuries do not seem, for instance, to determine sufficiently well the behavior of global [Ghil and Vautard, 1991] or local [Plaut et al., 1995] temperatures to permit a reliable climate forecast on the decadal timescale by this SSA MEM method [Ghil, 1997].

[216] On the seasonal-to-interannual timescale, it appears that the doubly periodic variability associated with the low-frequency and quasi-biennial ENSO modes is sufficiently large and regular to help prediction substantially. We saw in section 2.2 that these two modes capture $43 \%$ of the variance in the SO I time series and that their constructive interference accounts essentially for all strong and moderate EI Niños and La Niñas (see Figure 6). Table 4 shows that the SSA-MEM method performs as well as, or better than, two other statistical methods and three dynamical models, including a fully coupled GCM. This does not mean that dynamical ENSO models ought to be abandoned. It does mean, though, that they have to meet higher standards of forecast skill than beating the classical benchmark of "damped persistence," i.e., persistence gradually regressing to climatology.

[217] M ore important, the relatively high accuracy of the SSA-MEM forecast supports the essential role played by the two oscillatory modes, low-frequency and quasi-biennial, in E N SO variability. It emphasizes therewith the necessity for dynamical models to simulate the correct period, amplitude, and phase of these two modes. O nce a physical model does that, it can proceed to capture other aspects of ENSO dynamics, which are probably crucial in predicting the magnitude of extreme events, like the 1997-1998 EI Niño, or the extratropical aspects of seasonal-to-interannual climate variability. 
TABLE 4. Comparison of 6-Month-Lead Skill of ENSO Forecast Models [After Ghil and Jiang, 1998] ${ }^{a}$

\begin{tabular}{|c|c|c|c|c|c|c|}
\hline & \multicolumn{3}{|c|}{ Dynamical } & \multicolumn{3}{|c|}{ Statistical } \\
\hline & $\begin{array}{c}\text { Zebiak and Cane } \\
\text { [1987] }\end{array}$ & $\begin{array}{c}\text { Barnett et al. } \\
\text { [1993] }\end{array}$ & $\begin{array}{l}\text { Ji et al. } \\
\text { [1994] }\end{array}$ & $\begin{array}{c}\text { Barnston and } \\
\text { Ropelewski } \\
\text { [1992] }\end{array}$ & $\begin{array}{c}\text { Van den Dool } \\
\quad[1994]\end{array}$ & $\begin{array}{l}\text { Jiang et al. } \\
\text { [1995b] }\end{array}$ \\
\hline M odel & $\begin{array}{l}\text { physical: simple } \\
\text { coupled }\end{array}$ & $\begin{array}{l}\text { hybrid: physical } \\
\text { ocean model } \\
\text { coupled to a } \\
\text { statistical } \\
\text { atmosphere }\end{array}$ & $\begin{array}{l}\text { physical: } \\
\text { coupled } \\
\text { ocean- } \\
\text { atmosphere } \\
\text { GCM }\end{array}$ & $\begin{array}{l}\text { statistical: } \\
\text { canonical } \\
\text { correlation } \\
\text { analysis }\end{array}$ & $\begin{array}{l}\text { empirical: constructed } \\
\text { analogs }\end{array}$ & $\begin{array}{l}\text { statistical: SSA } \\
\text { and MEM }\end{array}$ \\
\hline $\begin{array}{l}\text { Predicted SST } \\
\text { region, for }\end{array}$ & Niño-3 & central Pacific & Niño-3.4 & Niño-3.4 & Niño-3.4 & Niño-3 \\
\hline $5^{\circ} \mathrm{N}-5^{\circ} \mathrm{S}$ & $90^{\circ}-150^{\circ} \mathrm{W}$ & $140^{\circ}-180^{\circ} \mathrm{W}$ & $120^{\circ}-170^{\circ} \mathrm{W}$ & $120^{\circ}-170^{\circ} \mathrm{W}$ & $120^{\circ}-170^{\circ} \mathrm{W}$ & $90^{\circ}-150^{\circ} \mathrm{W}$ \\
\hline $\begin{array}{l}\text { Period of record } \\
\text { Skill (1982-1993) }\end{array}$ & 1970-1993 & 1966-1993 & 1984-1993 & 1956-1993 & 1956-1993 & 1984-1993 \\
\hline Corr ${ }^{b}$ & 0.62 & 0.65 & 0.69 & 0.66 & 0.66 & 0.74 \\
\hline $\mathrm{RM} \mathrm{SE}^{\mathrm{c}}$ & 0.95 & 0.97 & 0.83 & 0.89 & 0.89 & 0.50 \\
\hline$S^{d}$ & 1.08 & 1.10 & 1.00 & 1.11 & 1.11 & 1.00 \\
\hline
\end{tabular}

${ }^{\text {aT }}$ he first three models are dynamical, while the last three are statistical. Columns 2-5 are based on information from Barnston et al. [1994]; see further description of models and validation methodology in their paper and by Ghil and Jiang [1998]. SSA-M EM forecasts (rightmost column) have continued to be equally skillful in real-time forecasting since their early prediction of the 1997-1998 EI N iño [Saunders et al., 1997].

${ }^{b}$ Correlation.

CR oot-mean-square error.

dStandard deviation.

[218] To conclude, spectral methods of time series analysis and prediction are intimately connected with the nonlinear dynamics of climate. Advances in the methodology have benefited from the deeper insights into the study of time series that dynamical systems theory provides. These advances are contributing in turn to a better understanding and prediction of climate phenomena and processes.

\section{APPENDIX A: SSA, SPATIAL EOF'S, AND THE KARHUNEN-LOEVE THEOREMS}

\section{A1. Spatial and Temporal EOFs}

[219] Table A 1 outlines the formal analogy between SSA and the principal component analysis (PCA) of variability in spatial fields. The key idea in both cases is to identify the main patterns of this variability, in decreasing order of the associated variance. This idea is carried out by diagonalizing the appropriate covariance or correlation matrix, as shown in Table A 1.
[220] The eigenvectors of this matrix are called empirical orthogonal functions (EOFs) in the meteorological literature. We refer to Preisendorfer [1988] for the long history of the meteorological and oceanographic applications and for references to the early work of I. H olmström, J. E. K utzbach, E. N. L orenz, and W. D. Sellers. In the fluid mechanics literature the whole analysis of turbulent fields by the principal component approach has become known as proper orthogonal decomposition (POD). A n excellent discussion and many more references are given in chapter 3 of Holmes et al. [1996].

[221] The rigorous mathematical foundation for the spectral representation of random fields is analogous to that of orthonormal bases for the solutions of the classical equations of mathematical physics [Courant and Hilbert, 1953, 1962]. In the case of random fields that possess finite first- and second-order moments, the counterpart of the linear partial differential operator being diagonalized is the two-point covariance operator. The appropriate theorems have been proven in the 1940s by Kosambi [1943], Loève [1945], and Karhunen

TABLE A1. Analogy Between Karhunen-Loève Decomposition in Space (Traditional or Spatial EOFs) and Time (Temporal EOFs)

\begin{tabular}{|c|c|}
\hline Spatial EOFs & $S S A$ \\
\hline $\begin{array}{c}\phi(x, t)=\sum A_{k}(t) \rho_{k}(x) \\
x \text {-space } \\
C_{\phi}(x, y)=\mathscr{E}\{\phi(x, \omega) \phi(y, \omega)\} \cong 1 / T \int_{0}^{T} \phi(x, t) \phi(y, t) d t^{\mathrm{a}} \\
\mathbf{C}_{\phi} \rho_{k}(x)=\lambda_{k} \rho_{k}(x)^{\mathrm{b}}\end{array}$ & $\begin{array}{c}X(t+s)=\sum_{s-\operatorname{lag}} A_{k}(t) \rho_{k}(s) \\
C_{X}(s)=\mathscr{E}\{X((\quad), \omega) X(s, \omega)\} \cong 1 / T \int_{0}^{T} X(t) X(t+s) \\
d t^{\mathrm{a}} \\
\mathbf{C}_{X} \rho_{k}(s) \stackrel{\lambda_{k} \rho_{k}(s)^{\mathrm{b}}}{ }\end{array}$ \\
\hline
\end{tabular}

${ }^{a}$ The expectation operator $\mathscr{E}$ represents averaging over a theoretical ensemble of realizations denoted by $\omega$. This is approximated by an average over epochs $t$. See text for the rest of the notation.

bThe covariance operators $C_{\phi}$ and $C_{X}$ are approximated in practice by the corresponding matrices $\mathbf{C}_{\phi}$ and $\mathbf{C}_{X}$. 
[1946]. Mathematically rigorous results in the time domain, rather than the spatial domain, assume that the univariate stochastic process is weakly stationary or stationary in the wide sense [see Hannan, 1960; Ghil and Taricco, 1997; Mallat, 1998]. W ide-sense stationarity requires the process to have the same finite mean and finite variance at each time $t$ and a lag-autocorrelation function that is finite for all lags and depends only on lag.

[222] The covariance matrices obtained when discretizing the spatially continuous random fields or timecontinuous processes of $K$ arhunen-L oève theory are often quite large. Because of their symmetry, the singular value decomposition (SVD) of these matrices is especially simple [Golub and Van Loan, 1996, section 2.5], and hence it is the method of choice for their diagonalization. SVD is, in fact, one of the most widely used algorithms in the whole of numerical linear algebra.

[223] It seems that Colebrook [1978] was the first one to observe in the oceanographic literature that the eigenvectors of the lag-correlation matrix are data-adaptive band-pass filters in the time domain. He used this observation to improve the spectral analysis of zooplankton records in the North A tlantic, grouped either by species or by area into "spatial" eigenvectors. Vautard and Ghil [1989] noticed the special role played by pairs of nearly equal eigenvalues that are associated with temporal EOFs in phase quadrature, as well as their connection to an efficient representation of anharmonic limit cycles.

[224] Barnett and Hasselmann [1979] proposed the use of spatial EOFs that incorporate a given number of lags for the prediction of atmospheric and oceanic fields in the tropical Pacific. Weare and Nasstrom [1982] coined the term extended E OF (EEOF) analysis for their use of PCA to study variability in a sliding time window of meteorological and oceanographic fields. Lau and Chan [1985] applied this analysis to outgoing longwave radiation data over the Indian and tropical Pacific oceans.

[225] All these EEOF applications used a small and fixed number of lags: two in the case of Lau and Chan [1985], three for Weare and Nasstrom [1982], and 10 or 12 for Barnett and Hasselmann's [1979] work. This restriction was essential because of these authors' retaining the full extent of the spatial information provided.

[226] Kimoto et al. [1991] and Plaut and Vautard [1994], on the other hand, were fully aware of the need to use variable windows in univariate SSA to ensure the statistical significance of the results, while capturing the main periods of interest. To permit the use of large and variable lag windows in multichannel SSA (M-SSA), they recommended prior compression of the spatial information. This compression can be carried out either by spatial averaging [Dettinger et al., 1995b; Jiang et al., 1995a] or by prefiltering that uses spatial EOF analysis [Plaut and Vautard, 1994; Moron et al., 1998].

[227] EEOF analysis and M-SSA are thus both extensions of the classical Karhunen-Loève approach to the

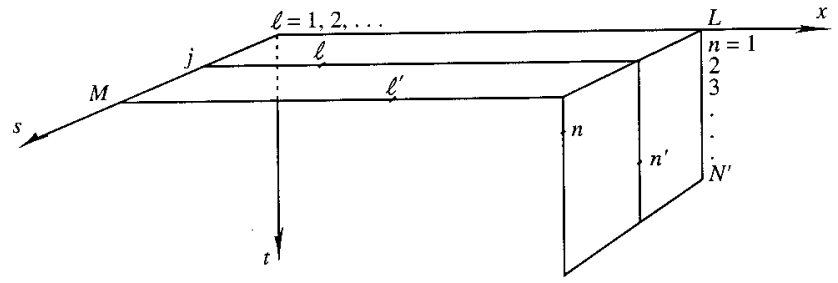

Figure A1. Comparison between PCA, SSA, and EEOFs or $M$-SSA. The axes are spatial coordinate (or spatial PC label) $x$, time $t$, and lag $s$; discrete values of these variables are labeled by $l, n$, and $j$, respectively (courtesy of $\mathrm{K} .-\mathrm{C} . \mathrm{M} \mathrm{O}$ ).

analysis of random fields and stationary processes. They both analyze, in one fell swoop, spatiotemporal variability of climatic fields, and both use, more often than not, the classical SVD algorithm of numerical linear algebra. EEOF analysis has its roots in and stays closer to spatial EOF analysis. M-SSA has its roots in univariate SSA and has been developing a battery of statistical significance tests for spatiotemporal variability inspired by those incorporated in the SSA-M TM Toolkit [Plaut and Vautard, 1994; Allen and Robertson, 1996].

\section{A2. Complementary Windows for M-SSA}

[228] Both univariate SSA and conventional PCA analysis are special cases of E E OF or M -SSA analysis: In SSA the number $L$ of channels equals 1 , while in PCA the number $M$ of lags is 1 . Following Allen and Robertson [1996], both algorithms can be understood in practice in terms of a "window." These windows are illustrated schematically in Figure A 1.

[229] Standard PCA slides a flat and narrow window, of length 1 and width $L$, over the data set's $N$ fields, each of which contains $L$ data points. PCA thus identifies the spatial patterns, i.e., the EOF s, which account for a high proportion of the variance in the $N$ views of the data set thus obtained. Equivalently, PCA can be described as sliding a long and narrow, $N \times 1$ window across the $L$ input channels and identifying high-variance temporal patterns, i.e., the PCs, in the corresponding $L$ views. In Figure $A 1$ the former view of things corresponds to sliding the $1 \times L$ window parallel to the $x$ axis along the $t$ axis. In the latter view one slides an $N \times 1$ window that starts out by lying along the $t$ axis parallel to the $x$ axis. In both cases, $M=1$.

[230] These two different window interpretations carry over into M-SSA. In the first case one proceeds from spatial PCA to EEOFs. To do so, we extend our $1 \times L$ window by $M$ lags to form an $M \times L$ window that lies in the horizontal $(x, s)$ plane of Figure A 1. By moving this window along the $t$ axis, we search for spatiotemporal patterns, i.e., the EEOFs, that maximize the variance in the $N^{\prime}=N-M+1$ overlapping views of the time series thus obtained. The EEOFs are the eigenvectors of the $L M \times L M$ lag-covariance matrix given by (48)-(50). 
[231] The second conceptual route leads from singlechannel SSA to M-SSA. To follow this route, we reduce the length of the $N \times 1$ window to $N^{\prime} \times 1$. This window still lies initially along the $t$ axis and we search for temporal patterns, i.e., the $N^{\prime}$-long PCs, that maximize the variance in the $M \times L$ views of the time series [Allen and Robertson, 1996; Robertson, 1996]. This is equivalent to SSA of the univariate time series formed by stringing together all the channels of the original multichannel time series end to end, with the complementary window $N^{\prime}$ playing the role of $M$ in SSA. The (reduced) PC S are the eigenvectors of the $N^{\prime} \times N^{\prime}$ reduced covariance matrix with the elements

$$
C_{j j^{\prime}}^{(R)}=\frac{1}{L M} \sum_{l=1}^{L}\left[\sum_{t=1}^{M} X_{l}(t+j-1) X_{l}\left(t+j^{\prime}-1\right)\right] .
$$

[232] This matrix, unlike the $L M \times L M$ matrix of (48), is given by

$$
\mathbf{C}^{(R)}=\frac{1}{L M} \tilde{\mathbf{X}} \tilde{\mathbf{X}}^{\mathrm{t}}
$$

In typical climate applications, $N^{\prime}<L M$; hence $\mathbf{C}^{(R)}$ is smaller than $\tilde{\mathbf{C}}_{\mathbf{X}}$ and the latter has a null space of dimension $L M-N^{\prime}$. The eigenvectors of $\tilde{\mathbf{C}}_{\mathbf{X}}$ that lie outside this null space, i.e., that correspond to nonzero eigenvalues, are identical to those of $\mathbf{C}^{(R)}$.

[233] ACKNOWLEDGMENTS. It is gratifying to thank our past and present SSA, MEM, and M TM collaborators, P. Billant, N. Jiang, C. L. Keppenne, M. Kimoto, J. M. Lees, K.-C. M O, J. Park, J. D. N eelin, J. M. Pap, M. C. Penland, G. Plaut, E. Simonnet, L. A. Smith, D. Sornette, C. M . Strong, C. Taricco, R. V autard, R. K. U Irich, Y. S. U nal and W. W eibel, for the pleasure of working together and exchanging ideas; we hope this review gives credit to the joint work and does not betray their occasionally different ideas. It is a pleasure to thank Tommy Dickey, past editor of Reviews of Geophysics, for inviting the paper and thus motivating us to bring it all together, and J effrey Park, Y ohan Guyado, and M ichael Perfit for their constructive comments on the original typescript. The dedication of $F$. Fleuriau in helping finalize the text is greatly appreciated, as is the help of $\mathrm{J}$. Meyerson with the figures. M.G.'s spectral methods work was supported by an NSF Special Creativity A ward. M.R.A. was supported by a NOAA Postdoctoral Fellowship in Climate and Global and Change (1994-1995), K.I. was supported by N A SA grant N A G 5-9294, D.K., F.V ., and the further development and maintenance of the SSA-MTM Toolkit were supported by NSF grant ATM 0082131, A.W.R. was supported by DOE grant DE-FG0398E R 62615, and P.Y. was supported by the French Commissariat à I'E nergie A tomique. M.G. is also indebted to the French A cadémie des Sciences for the Elf-A quitaine/CNRS Chair (1996) that provided the necessary leisure for the writing of this review's first draft and to his hosts over the years at the E cole Normale Supérieure (ENS) in Paris, the Département Terre-A tmosphère-O céan of the E NS, and the Laboratoire de Météorologie Dynamique du CNRS.

Thomas Torgersen was the Editor responsible for this paper.
He thanks J effrey J. Park for the technical review and M ichael Perfit and $Y$ ohan $G$ uyudo for cross-disciplinary reviews.

\section{REFERENCES}

A barbanel, H. D. I., Analysis of Observed Chaotic Data, 272 pp., Springer-V erlag, N ew Y ork, 1996.

A kaike, H., Fitting autoregressive models for prediction, Ann. Inst. Stat. Math., 21, 243-247, 1969.

A kaike, H., A new look at the statistical model identification, IEEE Trans. Autom. Control, 19, 716-723, 1974.

A llen, M ., Interactions between the atmosphere and oceans on time scales of weeks to years, Ph.D. thesis, 202 pp., U niv. of Oxford, Oxford, E ngland, 1992.

Allen, M.R ., and A. W. R obertson, D istinguishing modulated oscillations from coloured noise in multivariate data sets, Clim. Dyn., 12, 775-784, 1996.

Allen, M., and L. A. Smith, Investigating the origins and significance of low-frequency modes of climate variability, Geophys. Res. Lett., 21, 883- 886, 1994.

Allen, M. R ., and L. A. Smith, M onte Carlo SSA: D etecting irregular oscillations in the presence of coloured noise, J. Clim., 9, 3373-3404, 1996.

Allen, M. R., and L. A. Smith, Optimal filtering in singular spectrum analysis, Phys. Lett. A, 234, 419- 428, 1997.

Allen, M. R., P. Read, and L. Smith, Temperature timeseries?, Nature, 355, 686, 1992a.

Allen, M . R., P. R ead, and L. Smith, Temperature oscillations, Nature, 359, 679, 1992b.

A rneodo, A., F. A rgoul, J. F. M uzy, and M. Tabard, B eyond classical multifractal analysis using wavelets: U ncovering a multiplicative process hidden in the geometrical complexity of diffusion limited aggregates, Fractals, 1, 629-649, 1993.

A rnold, L., Stochastic Differential Equations: Theory and Applications, 228 pp., J ohn W iley, N ew Y ork, 1974.

A rnold, V. I., Ordinary Differential Equations, 280 pp., M IT Press, Cambridge, M ass., 1973.

A rnold, V. I., Geometrical Methods in the Theory of Ordinary Differential Equations, Springer-V erlag, N ew Y ork, 1983.

Bak, P., The D evil's staircase, Phys. Today, 39, 38- 45, 1986.

Barnett, T. P., and K. Hasselmann, Techniques of linear prediction, with application to oceanic and atmospheric fields in the tropical Pacific, Rev. Geophys., 17, 949-968, 1979.

Barnett, T. P., M . Latif, N. Graham, M. Flügel, S. Pazan, and W. White, ENSO and ENSO-related predictability, part I, Prediction of equatorial Pacific sea surface temperature with a hybrid coupled ocean-atmosphere model, J. Clim., 6, 1545-1566, 1993.

Barnston, A. G., and C. F. R opelewski, Prediction of E N SO episodes using canonical correlation analysis, J. Clim., 5, $1316-1345,1992$.

Barnston, A. G., et al., L ong-lead seasonal forecasts-Where do we stand?, Bull. Am. Meteorol. Soc., 75, 2097-2114, 1994.

Benoist, J. P., A nalyse spectrale de signaux glaciologiques: Etude des glaces sédimentaires déposées à D ome C, M orphologie du lit d'un glacier, Ph.D. thesis, U niv. of G renoble, Grenoble, France, 1986.

Berger, A. L., J. L. M elice, and L. Hinnov, A strategy for frequency spectra of Quaternary climate records, Clim. Dyn., 5, 227-240, 1991.

Birchfield, G. E., and M. G hil, Climate evolution in the Pliocene-Pleistocene as seen in deep sea $\delta^{18} 0$ records and in simulations: Internal variability versus orbital forcing, $J$. Geophys. Res., 98, 10,385-10,399, 1993.

Blackman, R. B., and J. W. Tukey, The Measurement of Power Spectra From the Point of View of Communication Engineering, D over, M ineola, N. Y ., 1958. 
Bloomfield, P., Fourier Analysis of Time Series: An Introduction, John Wiley, New Y ork, 1976.

Boiseau, M., M. G hil, and A. Juillet-Leclerc, Trends and interdecadal variability from south-central Pacific coral records, Geophys. Res. Lett., 26, 2881-2884, 1999.

Box, G. E. P., and G. M. J enkins, Time Series Analysis, Forecasting and Control, Holden-D ay, Boca R aton, Fla., 1970.

Broomhead, D. S., and G. P. King, Extracting qualitative dynamics from experimental data, Phys. D, 20, 217-236, 1986a.

Broomhead, D. S., and G. P. King, On the qualitative analysis of experimental dynamical systems, in Nonlinear Phenomena and Chaos, edited by S. Sarkar, pp. 113-144, Adam Hilger, Bristol, England, 1986b.

Broomhead, D. S., R. Jones, and G. P. King, Topological dimension and local coordinates from time series data, $J$. Phys. A, 20, 563-569, 1987.

Burg, J. P., Maximum entropy spectral analysis, paper presented at the 37th Annual International Meeting of the Society of Exploration Geophysicists, Oklahoma City, O kla., 1967. (Published in Modern Spectrum Analysis, edited by D. G. Childers, pp. 42-48, IE E E Press, Piscataway, N. J ., 1978.)

Chang, P., B. W ang, T. Li, and L. J i, Interactions between the seasonal cycle and the Southern Oscillation: Frequency entrainment and chaos in an intermediate coupled oceanatmosphere model, Geophys. Res. Lett., 21, 2817-2820, 1994.

Chao, Y., M. G hil, X . Li, and J. C. M cW illiams, Pacific interdecadal variability in this century's sea surface temperatures, Geophys. Res. Lett., 27, 2261-2264, 2000.

Chappellaz, J., J. M . B arnola, D. R aynaud, Y. S. Korotkevitch, and $C$. Lorius, Ice-core record of atmospheric methane over the past 160,000 years, Nature, 345, 127-131, 1990.

Chatfield, C., The Analysis of Time Series: An Introduction, 3rd ed., 286 pp., Chapman and H all, N ew Y ork, 1984.

Chen, F., and M. G hil, Interdecadal variablity of the thermohaline circulation and high-latitude surface fluxes, J. Phys. Oceanogr., 25, 2547-2568, 1995.

Chen, F., and M. G hil, Interdecadal variability in a hybrid coupled ocean-atmosphere model, J. Phys. Oceanogr., 26, 1561-1578, 1996.

Childers, D. G. (Ed.), Modern Spectrum Analysis, 331 pp., IEEE Press, Piscataway, N. J., 1978.

Cochrane, D., and G. H. Orcutt, A pplication of least squares regression to relationships containing autocorrelated error terms, J. Am. Stat. Assoc., 44, 32-61, 1949.

Colebrook, J. M., Continuous plankton records: Z ooplankton and environment, Northeast Atlantic and North Sea, Oceanol. Acta, 1, 9-23, 1978.

Constantin, P., C. Foias, B. Nicolaenko, and R. Temam, Integral Manifolds and Inertial Manifolds for Dissipative Partial Differential Equations, 122 pp., Springer-V erlag, N ew Y ork, 1989.

Cook, E. R ., B. M. Buckley, and R. D. D 'A rrigo, Interdecadal temperature oscillations in the Southern H emisphere: $\mathrm{E} v$ idence from Tasmanian tree rings since $300 \mathrm{~B}$. C., in $\mathrm{Na}$ tional Natural Climate Variability on Decade-to-Century Time-Scales, edited by D. G. M artinson et al., pp. 523-532, N atl. A cad. Press, W ashington, D. C., 1995.

Courant, R ., and D. H ilbert, Methods of Mathematical Physics, vol. I, 560 pp., W iley-I nterscience, N ew Y ork, 1953.

Courant, R ., and D. H ilbert, Methods of Mathematical Physics, vol. II, 830 pp., W iley-Interscience, N ew Y ork, 1962.

Daubechies, I., Ten Lectures on Wavelets, Soc. for Ind. and A ppl. M ath., Philadelphia, Pa., 1992.

Delprat, N., B. E scudé, P. G uillemain, R. K ronland-M artinet, P. T chamitchian, and B. Torrésani, A symptotic wavelet and
Gabor analysis: Extraction of instantaneous frequencies, IEEE Trans. Inf. Theory, 38, 644-664, 1992.

Delworth, T., S. M anabe, and R. Stouffer, Interdecadal variations of the thermohaline circulation in a coupled oceanatmosphere model, J. Clim., 6, 1993-2011, 1993.

Dettinger, M. D., and M. Ghil, Seasonal and interannual variations of atmospheric $\mathrm{CO}_{2}$ and climate, Tellus, Ser. B, 50, 1-24, 1998.

Dettinger, M. D., M. G hil, C. M. Strong, W. W eibel, and P. Y iou, Software expedites singular-spectrum analysis of noisy time series, Eos Trans. $A G U, 76(2), 12,14,21,1995 a$. (V ersion 4.0 available as freeware on the World W ide W eb at http://www.atmos.ucla.edu/tcd.)

Dettinger, M. D., M . G hil, and C. Keppenne, Interannual and interdecadal variability in $U$ nited States surface-air temperatures, 1910-1987, Clim. Change, 31, 35-66, 1995b.

Dijkstra, H., and J. Molemaker, Imperfections of the North A tlantic wind-driven ocean circulation: Continental geometry and wind stress, J. Mar. Res., 57, 1-28, 1999.

D razin, P. G ., and G. P. King, Introduction, Phys. D, 58, 7-11, 1992.

Eckmann, J.-P., R oads to turbulence in dissipative dynamical systems, Rev. Mod. Phys., 53, 643-654, 1981.

Eckmann, J.-P., and D. R uelle, E rgodic theory of chaos and strange attractors, Rev. Mod. Phys., 57, 617-656, 1985. (Correction, Rev. Mod. Phys., 57, 1115, 1985.)

E gger, J., PO Ps and M OPs, Clim. Dyn., 15, 561-568, 1999.

Elsner, J. B., and A. A. T sonis, Do bidecadal oscillations exist in the global temperature record?, Nature, 353, 551-553, 1991.

Farge, M., W avelet transforms and their applications to turbulence, Annu. Rev. Fluid Mech., 24, 395- 457, 1992.

Farmer, J. D., E. Ott, and J. A. Y orke, The dimension of chaotic attractors, Phys. D, 7, 153-180, 1983.

Feigenbaum, M. J., Q uantitative universality for a class of non-linear transformations, J. Stat. Phys., 19, 25-52, 1978.

Feigenbaum, M. J., L. P. K adanoff, and S. J. Shenker, Q uasiperiodicity in dissipative systems: A renormalization group analysis, Phys. D, 5, 370-386, 1982.

Feller, W., An Introduction to Probability Theory and Its Applications, 3rd ed., vol. I, 509 pp., J ohn W iley, N ew Y ork, 1968.

Feller, W., An Introduction to Probability Theory and Its Applications, 2nd ed., vol. II, 699 pp., J ohn Wiley, N ew Y ork, 1971.

Flandrin, P., Time-Frequency/Time-Scale Analysis, 386 pp., A cademic, San Diego, Calif., 1999.

Foias, C., A. E. Frazho, and P. J. Sherman, A geometric approach to the maximum likelihood spectral estimator of sinusoids in noise, IEEE Trans. Inf. Theory, 34, 1066-1070, 1988.

Fraedrich, K., Estimating the dimension of weather and climate attractors, J. Atmos. Sci., 43, 419-432, 1986.

Fraedrich, K., and $\mathrm{H}$. Boettger, A wavenumber-frequency analysis of the $500 \mathrm{mb}$ geopotential at 50N, J. Atmos. Sci., 35, 745-750, 1978.

Gabor, D., Theory of communication, J. Inst. Electr. Eng., 93, 429-457, 1946.

G hil, M., Cryothermodynamics: The chaotic dynamics of paleoclimate, Phys. D, 77, 130-159, 1994.

G hil, M., The SSA-M TM toolkit: A pplications to analysis and prediction of time series, Proc. SPIE Int. Soc. Opt. Eng., 3165, 216-230, 1997.

G hil, M ., and S. Childress, Topics in Geophysical Fluid Dynamics: Atmospheric Dynamics, Dynamo Theory and Climate Dynamics, 485 pp., Springer-V erlag, N ew Y ork, 1987.

Ghil, M., and N. Jiang, R ecent forecast skill for the EI NiñoSouthern O scillation, Geophys. Res. Lett., 25, 171-174, 1998.

G hil, M ., and K. C. M o, Intraseasonal oscillations in the global 
atmosphere, part I, Northern H emisphere and tropics, $J$. Atmos. Sci., 48, 752-779, 1991a.

G hil, M ., and K. C. M o, Intraseasonal oscillations in the global atmosphere, part II, Southern Hemisphere, J. Atmos. Sci., 48, 780-790, 1991b.

G hil, M ., and A. W . R obertson, Solving problems with GCM s: General circulation models and their role in the climate modeling hierarchy, in General Circulation Model Development: Past, Present and Future, edited by D. R andall, pp. 285-325, A cademic, San Diego, Calif., 2000.

G hil, M ., and C. Taricco, A dvanced spectral analysis methods, in Past and Present Variability of the Solar-Terrestrial System: Measurement, Data Analysis and Theoretical Models, edited by G. C. Castagnoli and A. Provenzale, pp. 137-159, Soc. Ital. di Fis., Bologna, Italy, 1997.

G hil, M., and R. V autard, Interdecadal oscillations and the warming trend in global temperature time series, Nature, 350, 324-327, 1991.

Ghil, M ., and P. Y iou, Spectral methods: What they can and cannot do for climatic time series, in Decadal Climate Variability: Dynamics and Predictability, edited by D. A nderson and J. Willebrand, pp. 445-481, Springer-V erlag, N ew York, 1996.

Ghil, M., M. Kimoto, and J. D. Neelin, Nonlinear dynamics and predictability in the atmospheric sciences, U.S. Natl. Rep. Int. Union Geod. Geophys. 1987-1991, Rev. Geophys., 29, 46-55, 1991.

Gilman, D. L., F. J . Fuglister, and J . J . M itchell, On the power spectrum of "red noise," J. Atmos. Sci., 20, 182-184, 1963.

Gleick, J., Chaos: Making a New Science, 354 pp., Viking, N ew Y ork, 1987.

Golub, G. H., and C. F. V an Loan, Matrix Computations, 3rd ed., 642 pp., Johns Hopkins U niv. Press, Baltimore, M d., 1996.

G rassberger, $\mathrm{P}$., and I. Procaccia, $M$ easuring the strangeness of strange attractors, Phys. D, 9, 189-208, 1983.

Guckenheimer, J., and P. Holmes, Nonlinear Oscillations, Dynamical Systems and Bifurcations of Vector Fields, 459 pp., Springer-V erlag, N ew Y ork, 1983.

Hannan, E. J., Time Series Analysis, 152 pp., Methuen, New Y ork, 1960.

Hannan, E.J., Multiple Time Series, 536 pp., J ohn W iley, N ew Y ork, 1970.

Hasan, T., Complex demodulation: Some theory and applications, in Handbook of Statistics 3: Time Series in the Frequency Domain, edited by D. R. Brillinger and P. R. K rishnaiah, pp. 125-156, N orth H olland, N ew Y ork, 1983.

H asselmann, K., Stochastic climate models, Tellus, 6, 473- 485, 1976.

Hasselmann, K., PIPs and POPs: The reduction of complex dynamical systems using principal interaction and principal oscillation patterns, J. Geophys. Res., 93, 11,015-11,021, 1988.

H aykin, S., and S. Kessler, Prediction-error filtering and maximum-entropy spectral estimation, in Nonlinear Methods of Spectral Analysis, edited by S. H aykin, pp. 9-72, SpringerVerlag, N ew Y ork, 1983.

Holmes, P., J. L. L umley, and G. Berkooz, Turbulence, Coherent Structures, Dynamical Systems and Symmetry, Cambridge U niv. Press, N ew Y ork, 1996.

Intergovernmental Panel on Climate Change, Climate Change: The IPCC Scientific Assessment, edited by J. T. H oughton, G. J . J enkins, and J . J. E phraums, 365 pp., Cambridge U niv. Press, New Y ork, 1990.

Jenkins, G. M., and D. G. Watts, Spectral Analysis and Its Applications, Holden-D ay, Boca R aton, Fla., 1968.

Ji, M., A. K umar, and A. L eetmaa, A n experimental coupled forecast system at the National Meteorological Center: Some early results, Tellus, Ser. A, 46, 398-418, 1994.
J iang, N., D. Neelin, and M. G hil, Quasi-quadrennial and quasi-biennial variability in the equatorial Pacific, Clim. Dyn., 12, 101-112, 1995a.

J iang, N., M. Ghil, and D. Neelin, Forecasts of equatorial Pacific SST anomalies by using an autoregressive process and singular spectrum analysis, in Experimental Long-Lead Forecasts Bulletin, vol. 4, no. 1, pp. 24-27, Natl. Cent. for Environ. Predict., Camp Springs, M d., 1995b.

J iang, S., F .-F. J in, and M. G hil, M ultiple equilibria, periodic, and aperiodic solutions in a wind-driven, double-gyre, shallow-water model, J. Phys. Oceanogr., 25, 764-786, 1995c.

J in, F.-F., J. D. Neelin, and M. G hil, EI Niño on the Devil's staircase: A nnual subharmonic steps to chaos, Science, 264, 70-72, 1994.

J in, F.-F., J. D. Neelin, and M. G hil, EI Niño/Southern O scillation and the annual cycle: Subharmonic frequency-locking and aperiodicity, Phys. D, 98, 442-465, 1996.

J ouzel, J., et al., Extending the Vostok ice-core record of paleoclimate to the penultimate glacial period, Nature, 364, 407- 412, 1993.

Kaplan, J. L., and J. A. Y orke, Chaotic behavior of multidimensional difference equations, in Lect. Not. in Math., vol. 730, Functional Differential Equations and Approximation of Fixed Points, edited by H. O . Peitgen and H. O. W alther, pp. 228-237, Springer-V erlag, N ew Y ork, 1979.

Karhunen, K., Zur Spektraltheorie stochastischer Prozesse, Ann. Acad. Sci. Fenn. Ser. A1, Math. Phys., 34, 1946.

Kay, S. M ., Modern Spectral Analysis: Theory and Applications, 543 pp., Prentice-H all, Old Tappan, N.J ., 1988.

Kendall, M., and A. Stuart, The Advanced Theory of Statistics, vol. 2, 4th ed., M acmillan, Old Tappan, N. J., 1977.

Keppenne, C. L., and M. G hil, A daptive filtering and prediction of the Southern O scillation Index, J. Geophys. Res., 97, 20,449-20,454, 1992.

Keppenne, C. L., and M. G hil, A daptive filtering and prediction of noisy multivariate signals: A $n$ application to subannual variability in atmospheric angular momentum, Int. J. Bifurc. Chaos, 3, 625- 634, 1993.

Kimoto, M., and M. G hil, M ultiple flow regimes in the N orthern Hemisphere winter, part II, Sectorial regimes and preferred transitions, J. Atmos. Sci., 50, 2645-2673, 1993.

Kimoto, M., M. Ghil, and K. C. Mo, Spatial structure of the extratropical 40-day oscillation, in Proceedings of the 8th Conference on Atmospheric and Oceanic Waves and Stability, pp. 115-116, A m. M eteorol. Soc., Boston, M ass., 1991.

Koch, D. M ., and M. E. M ann, Spatial and temporal variability of ${ }^{7}$ Be surface concentration, Tellus, Ser. B, 48, 387-398, 1996.

Kosambi, D. D., Statistics in function space, J. Indian Math. Soc., 7, 76-88, 1943.

Kumar, P., and E. Foufoula-G eorgiou, W avelet analysis for geophysical applications, Rev. Geophys., 35, 385- 412, 1997.

Kumaresan, R., and D. W. Tufts, Data-adaptive principal component signal processing, in Proceedings of the Conference on Decision and Control, pp. 949-954, IEEE Press, Piscataway, N. J ., 1980.

Kuo, C., C. Lindberg, and D. J. Thomson, Coherence established between atmospheric carbon dioxide and global temperature, Nature, 343, 709-713, 1990.

Lall, U ., and M. M ann, The Great Salt Lake: A barometer of low-frequency climatic variability, Water Resour. Res., 31, 2503-2515, 1995.

Lasota, A., and M. C. M ackey, Chaos, Fractals, and Noise: Stochastic Aspects of Dynamics, 2nd ed., Springer-V erlag, New Y ork, 1994

L atif, M ., and T. Barnett, Causes of decadal climate variability over the North Pacific and North A merica, Science, 266, 634- 637, 1994.

Lau, K. M., and P. H. Chan, A spects of the 40-50 day oscil- 
lation during the northern winter as inferred from outgoing longwave radiation, Mon. Weather Rev., 113, 1889-1909, 1985.

Lees, J. M ., R eshaping spectrum estimates by removing periodic noise: A pplication to seismic spectral ratios, Geophys. Res. Lett., 22, 513-516, 1995.

Legras, B., and M. G hil, Persistent anomalies, blocking and variations in atmospheric predictability, J. Atmos. Sci., 42, 433- 471, 1985.

Leith, C. E., Climate response and fluctuation dissipation, $J$. Atmos. Sci., 32, 2022-2026, 1975.

Lichtenberg, A.J., and M. A . Lieberman, Regular and Chaotic Dynamics, 692 pp., 2nd ed., Springer-V erlag, N ew Y ork, 1992.

L oève, M ., Fonctions aléatoires de second ordre, C. R. Acad. Sci. Paris, 220, 380, 1945.

Lorenz, E. N ., D eterministic nonperiodic flow, J. Atmos. Sci., 20, 130-141, 1963.

Lorenz, E. N ., A tmospheric predictability as revealed by naturally occurring analogues, J. Atmos. Sci., 26, 636-646, 1969.

M acD onald, G. J ., Spectral analysis of time series generated by nonlinear processes, Rev. Geophys., 27, 449-469, 1989.

M allat, S., A Wavelet Tour of Signal Processing, 577 pp., A cademic, San Diego, Calif., 1998.

Mallat, S., and Z. Zhang, Matching pursuit with time-frequency dictionaries, IEEE Trans. Signal Process., 41, 33973415, 1993.

M andelbrot, B. B., The Fractal Geometry of Nature, 2nd ed., 460 pp., W. H. Freeman, N ew Y ork, 1982.

$M$ añé, R., O n the dimension of the compact invariant sets of certain non-linear maps, in Lect Not. in Math., vol. 898, Dynamical Systems and Turbulence, edited by D. A. R and and L.-S. Y oung, pp. $230-242$, Springer-V erlag, N ew Y ork, 1981.

M ann, M . E ., and J . M . L ees, R obust estimation of background noise and signal detection in climatic time series, Clim. Change, 33, 409-445, 1996.

M ann, M. E ., and J. Park, Spatial correlations of interdecadal variation in global surface temperatures, Geophys. Res. Lett., 20, 1055-1058, 1993.

Mann, M. E., and J. Park, G lobal scale modes of surface temperature variability on interannual to century timescales, J. Geophys. Res., 99, 25,819-25,833, 1994.

M ann, M . E ., and J. Park, G reenhouse warming and changes in the seasonal cycle of temperature: M odel versus observations, Geophys. Res. Lett., 23, 1111-1114, 1996a.

Mann, M. E., and J. Park, Joint spatiotemporal modes of surface temperature and sea level pressure variablity in the Northern Hemisphere during the last century, J. Clim., 9, 2137-2162, 1996b.

Mann, M. E., and J. Park, O scillatory spatiotemporal signal detection in climate studies: A multiple-taper spectral domain approach, Adv. Geophys., 41, 1-131, 1999.

M ann, M . E., U . L all, and B. Saltzman, D ecadal- to centennialscale climate variability: Insights into the rise and fall of the G reat Salt Lake, Geophys. Res. Lett., 22, 937-940, 1995a.

M ann, M. E., J. Park, and R. S. Bradley, G lobal interdecadal and century-scale climate oscillations during the past five centuries, Nature, 378, 266-270, 1995b.

M eyer, Y., Wavelets and Operators, 223 pp., Cambridge U niv. Press, N ew Y ork, 1992.

M eyer, Y., Wavelets: Algorithms and Applications, 133 pp., Soc. for Ind. and A ppl. M ath., Philadelphia, Pa., 1993.

M eyers, S. D., B. G. Kelly, and J . J . O'Brien, A n introduction to wavelet analysis in oceanography and meteorology, with application to the dispersion of $Y$ anai waves, Mon. Weather Rev., 121, 2858-2866, 1993.
Mitchell, J. M., An overview of climatic variability and its causal mechanisms, Quat. Res., 6, 481- 493, 1976.

M ommersteeg, H., M. F. L outre, R. Y oung, T. A . Wijmstra, and $\mathrm{H}$. Hooghiemstra, Orbital forced frequencies in the 975,000 year pollen record from Tenagi Philippon (G reece), Clim. Dyn., 11, 4-24, 1995.

M oron, V ., R. V autard, and M. G hil, Trends, interdecadal and interannual oscillations in global sea-surface temperatures, Clim. Dyn., 14, 545-569, 1998.

Neelin, J. D., A hybrid coupled general circulation model for EI Niño studies, J. Atmos. Sci., 47, 674-693, 1990.

Neelin, J. D., M. Latif, and F.-F. J in, Dynamics of coupled ocean-atmosphere models: The tropical problem, Annu. Rev. Fluid Mech., 26, 617-659, 1994.

Neelin, J. D., D. S. Battisti, A . C. H irst, F.-F. J in, Y. W akata, T. Y amagata, and S. E. Z ebiak, E N SO theory, J. Geophys. Res., 103, 14,261-14,290, 1998.

O oms, M ., Empirical Vector Autoregressive Modeling, SpringerVerlag, N ew Y ork, 1988.

Ott, E., T. Sauer, and J. A. Y orke (E ds.), Coping with Chaos: Analysis of Chaotic Data and the Exploitation of Chaotic Systems, 418 pp., John W iley, New Y ork, 1994.

Packard, N. H ., J. P. Crutchfield, J. D . Farmer, and R. S. Shaw, Geometry from a time series, Phys. Rev. Lett., 45, 712-716, 1980.

Paluš, M., and I. D vořák, Singular value decomposition in attractor reconstruction: Pitfalls and precautions, Phys. D, 55, 221-234, 1992.

Park, J., Envelope estimation for quasi-periodic geophysical signals in noise: A multitaper approach, in Statistics in the Environmental and Earth Sciences, edited by A. T. W alden and P. Guttorp, pp. 189-219, Edward A rnold, London, 1992.

Park, J., and K. A. M aasch, Plio-Pleistocene time evolution of the 100-kyr cycle in marine paleoclimate records, J. Geophys. Res., 98, 447-461, 1993.

Park, J., and M. E. M ann, Interannual temperature events and shifts in global temperature: A multiwavelet correlation approach, Earth Interact., 4, 2000. (A vailable at http:// E arthl nteractions.org)

Park, J., C. R. Lindberg, and F. L. I. Vernon, M ultitaper spectral analysis of high-frequency seismograms, J. Geophys. Res., 92, 12,675-12,684, 1987.

Penland, C., R andom forcing and forecasting using principal oscillation pattern analysis, Mon. Weather Rev., 117, 21652185, 1989.

Penland, C., A stochastic model of IndoPacific sea surface temperature anomalies, Phys. D, 98, 534-558, 1996.

Penland, C., and M. G hil, Forecasting Northern Hemisphere 700-mb geopotential height anomalies using empirical normal modes, Mon. Weather Rev., 121, 2355-2372, 1993.

Penland, C., M. G hil, and K. W eickmann, A daptive filtering and maximum entropy spectra, with application to changes in atmospheric angular momentum, J. Geophys. Res., 96, 22,659-22,671, 1991.

Percival, D. B., and A. T. Walden, Spectral Analysis for Physical Applications, 583 pp., Cambridge U niv. Press, New Y ork, 1993.

Pike, E. R., J. G. M cWhirter, M. Bertero, and C. de Mol, Generalized information theory for inverse problems in signal processing, IEE Proc., 131, 660-667, 1984.

Plaut, G., and R. V autard, Spells of low-frequency oscillations and weather regimes in the Northern Hemisphere, $J$. Atmos. Sci., 51, 210-236, 1994.

Plaut, G., M. G hil, and R. V autard, Interannual and interdecadal variability in 335 years of central England temperatures, Science, 268, 710-713, 1995.

Preisendorfer, R. W., Principal Component Analysis in Meteo- 
rology and Oceanography, 425 pp., E Isevier Sci., N ew Y ork, 1988.

Press, W. H., B. P. Flannery, S. A. Teukolski, and W. T. V ettering, Numerical Recipes: The Art of Scientific Computing, 818 pp., Cambridge U niv. Press, N ew Y ork, 1988.

Priestley, M. B., Spectral Analysis and Time Series, vol. 1, Univariate Series, 653 pp., Academic, San Diego, Calif., 1981a.

Priestley, M. B., Spectral Analysis and Time Series, vol. 2, Multivariate Series, Prediction and Control, pp. 654-890, A cademic, San D iego, Calif., 1981b.

Quinn, T. M., F. W. Taylor, and T. J. Crowley, A 173 year stable isotope record from a tropical South Pacific coral, Quat. Sci. Rev., 12, 407-418, 1993.

Quon, C., and M. G hil, M ultiple equilibria and stable oscillations in thermosolutal convection at small aspect ratio, $J$. Fluid Mech., 291, 33-56, 1995.

R ahmstorf, S., Bifurcation of the A tlantic thermohaline circulation in response to changes in the hydrological cycle, Nature, 378, 145-149, 1995.

Rasmusson, E. M., X. Wang, and C. F. Ropelewski, The biennial component of ENSO variability, J. Mar. Syst., 1, 71-96, 1990.

R ayner, N. A ., C. K . Folland, D. E . Parker, and E. B. H orton, A new global sea-ice and sea surface temperature (GISST) data set for 1903-1994 for forcing climate models, Hadley Cent. Intern. Not. 69, 13 pp., 1995.

Robertson, A. W., Interdecadal variability over the North Pacific in a multi-century climate simulation, Clim. Dyn., 12, 227-241, 1996.

Robertson, A. W., and C. R. M echoso, Interannual and decadal cycles in river flows of southeastern South A merica, J. Clim., 11, 2570-2581, 1998.

Robertson, A. W., and C. R. M echoso, Interannual and interdecadal variability of the South Atlantic Convergence Zone, Mon. Weather Rev., 128, 2947-2957, 2000.

Robertson, A. W., C.-C. M a, C. R. M echoso, and M. Ghil, Simulation of the tropical Pacific climate with a coupled ocean-atmosphere general circulation model, part I, The seasonal cycle, J. Clim., 8, 1178-1198, 1995a.

Robertson, A. W., C.-C. M a, M. G hil, and C. R. M echoso, Simulation of the tropical Pacific climate with a coupled ocean-atmosphere general circulation model, part II, I nterannual variability, J. Clim., 8, 1199-1216, 1995b.

Ropelewski, C. F., and P. D. Jones, An extension of the Tahiti-Darwin Southern Oscillation index, Mon. Weather Rev., 115, 2161-2165, 1987.

R oux, J. C., A. R ossi, S. B achelart, and C. V idal, R epresentation of a strange attractor from an experimental study of chemical turbulence, Phys. Lett. A, 77, 391-393, 1980.

R uelle, D., Small random perturbations of dynamical systems and the definition of attractors, Commun. Math. Phys., 82, 137-151, 1981.

R uelle, D., Deterministic chaos: The science and the fiction, Proc. R. Soc. London, Ser. A, 427, 241-248, 1990.

Ruelle, D., and F. Takens, On the nature of turbulence, Commun. Math. Phys., 20, 167-192, 1971. (Correction, Commun. Math. Phys., 23, 343-344, 1971.)

Sauer, T., J. A. Y orke, and M. Casdagli, E mbedology, J. Stat. Phys., 65, 579- 616, 1991.

Saunders, A ., and M. G hil, A Boolean delay equation model of ENSO variability, Phys. D, 160, 54-78, 2001.

Saunders, A., M. G hil, and J. D. Neelin, F orecasts of Niño-3 SST anomalies and SOI based on singular spectrum analysis combined with the maximum entropy method (1997-2000), in Experimental Long-Lead Forecasts Bulletin, vol. 6, no. 3, pp. 43- 44, N atl. Cent. for Environ. Predict., Camp Springs, M d., 1997.
Schuss, Z., Theory and Applications of Stochastic Differential Equations, 321 pp., John W iley, N ew Y ork, 1980.

Schuster, A ., On the investigation of hidden periodicities with application to a supposed 26-day period of meteorological phenomena, J. Geophys. Res., 3, 13-41, 1898.

Schuster, H. G., Deterministic Chaos: An Introduction, 2nd ed., 270 pp., Physik-V erlag, W einheim, Germany, 1988.

Shannon, C. E., Communication in the presence of noise, Proc. IRE, 37, 10-21, 1949.

Slepian, S., Prolate spheroidal wave functions, F ourier analysis and uncertainty, V , The discrete case, Bell. Syst. Tech. J., 57, 1371-1430, 1978.

Smale, S., Differentiable dynamical systems, Bull. Am. Math. Soc., 73, 199-206, 1967.

Speich, S., H. Dijkstra, and M. G hil, Successive bifurcations in a shallow-water model, applied to the wind-driven ocean circulation, Nonlinear Process. Geophys., 2, 241-268, 1995.

Stoker, J . J., Nonlinear Vibrations in Mechanical and Electrical Systems, 273 pp., W iley-I nterscience, N ew Y ork, 1950.

Strang, G., Wavelets and dilation equations: A brief introduction, SIAM Rev., 31(4), 614-627, 1989.

Sutton, R . T ., and M . R. A llen, D ecadal predictability of N orth A tlantic sea surface temperature and climate, Nature, 388, 563-567, 1997.

Takens, F., D etecting strange attractors in turbulence, in Lect. Not. in Math., vol. 898, Dynamical Systems and Turbulence, edited by D. A. R and and L.-S. Y oung, pp. 366-381, Springer-V erlag, N ew Y ork, 1981.

Temam, R., Infinite-Dimensional Dynamical Systems in Mechanics and Physics, 2nd ed., 648 pp., Springer-V erlag, N ew Y ork, 1997.

Thomson, D. J., Spectrum estimation and harmonic analysis, Proc. IEEE, 70, 1055-1096, 1982.

Thomson, D. J., Q uadratic-inverse spectrum estimates: A pplications to palaeoclimatology, Philos. Trans. R. Soc. London, Ser. A, 332, 539-597, 1990a.

Thomson, D. J ., Time series analysis of $\mathrm{H}$ olocene climate data, Philos. Trans. R. Soc. London, Ser. A, 330, 601-616, 1990b.

Thomson, D. J ., The seasons, global temperature, and precession, Science, 268, 59-68, 1995.

Thomson, D. J., and A. D. Chave, Jackknifed error estimates for spectra, coherences, and transfer functions, in Advances in Spectrum Analysis, edited by S. Haykin, chap. 2, pp. 58-113, Prentice-H all, Old Tappan, N. J., 1990.

Torrence, C., and G. P. Compo, A practical guide to wavelet analysis, Bull. Am. Meteorol. Soc., 79, 61-78, 1998.

Tziperman, E., L. Stone, M. A . Cane, and H. Jarosh, EI N iño chaos: Overlapping of resonances between the seasonal cycle and the Pacific ocean-atmosphere oscillator, Science, 264, 72-74, 1994

U nal, Y. S., and M. G hil, Interannual and interdecadal oscillation patterns in sea level, Clim. Dyn., 11, 255-278, 1995.

$\mathrm{V}$ an den $\mathrm{D}$ ool, H. M ., Searching for analogues: H ow long must we wait?, Tellus, Ser. A, 46, 314-324, 1994.

$\mathrm{V}$ an der Pol, B., Biological rhythms considered as relaxation oscillations, Acta Med. Scand. Suppl., 108, 76-87, 1940.

V autard, R ., and M. G hil, Singular spectrum analysis in nonlinear dynamics, with applications to paleoclimatic time series, Phys. D, 35, 395-424, 1989.

V autard, R., P. Y iou, and M. G hil, Singular spectrum analysis: A toolkit for short noisy chaotic signals, Phys. D, 58, 95-126, 1992.

Venegas, S. A., L. A. M ysak, and D. N. Straub, A tmosphereocean coupled variability in the South A tlantic, J. Clim., 10, 2904-2920, 1997

V on Storch, H., and F. W. Z wiers, Statistical Analysis in Climate Research, 484 pp., Cambridge U niv. Press, N ew Y ork, 1999.

V on Storch, H., T. Bruns, I. Fischer-Bruns, and K. H assel- 
mann, Principal oscillation pattern analysis of the 30 - to 60 -day oscillation in general circulation model equatorial troposphere, J. Geophys. Res., 93, 11,022-11,036, 1988.

Walker, G., On periodicity in series of related terms, Proc. $R$. Soc. London, Ser. A, 131, 518-532, 1931.

W ang, B., and $Y$. W ang, Temporal structure of the Southern O scillation as revealed by waveform and wavelet analysis, J. Clim., 9, 1586-1598, 1996.

W eare, B. C., and J. N. N asstrom, Examples of extended empirical orthogonal function analyses, Mon. Weather Rev., 110, 784-812, 1982.

W eaver, A . J ., J . M arotzke, P. F. Cummins, and E . S. Sarachik, Stability and variability of the thermohaline circulation, $J$. Phys. Oceanogr., 23, 39-60, 1993.

Weng, H.-Y., and K.-M . Lau, W avelets, period-doubling and time-frequency localization with application to satellite data analysis, J. Atmos. Sci., 51, 2523-2541, 1994.

Whitney, B., Differentiable manifolds, Ann. Math., 37, 645680, 1936.

Whittle, P., The simultaneous estimation of a time series harmonic component and covariance structure, Trab. Estadist, 3, 43-57, 1952.

W iener, N., Extrapolation, Interpolation and Smoothing of Stationary Time Series, With Engineering Applications, 163 pp., M IT Press, Cambridge, M ass., 1949.

Wiggins, S., Global Bifurcations and Chaos (Analytical Methods), 494 pp., Springer-V erlag, N ew Y ork, 1988.

Y iou, P., Dynamique du paléoclimat: Des données et des modèles, Ph.D . thesis, 183 pp., U niv. Pierre et M arie Curie, Paris, 1994.

Y iou, P., C. Genthon, J. J ouzel, M. G hil, H. Le Treut, J. M. Barnola, C. L orius, and Y. N. Korotkevitch, H igh-frequency paleovariability in climate and in $\mathrm{CO}_{2}$ levels from $\mathrm{V}$ ostok ice-core records, J. Geophys. Res., 96, 20,365-20,378, 1991.

Y iou, P., M. Ghil, J. Jouzel, D. Paillard, and R. V autard, Nonlinear variability of the climatic system, from singular and power spectra of L ate Q uaternary records, Clim. Dyn., 9, 371-389, 1994.

Y iou, P., J. J ouzel, S. J ohnsen, and Ö. E. Rögnvaldsson, R apid oscillations in Vostok and GRIP ice cores, Geophys. Res. Lett., 22, 2179-2182, 1995.

Y iou, P., M. F. Loutre, and E. Baert, Spectral analysis of climate data, Surv. Geophys., 17, 619-663, 1996.

Y iou, P., K. Fuhrer, L. D. M eeker, J . J ouzel, S. J ohnsen, and P. A. M ayewski, Paleoclimatic variability inferred from the spectral analysis of G reenland and A ntarctic ice core data, J. Geophys. Res., 102, 26,441-26,454, 1997.

Y iou, P., D. Sornette, and M. G hil, D ata-adaptive wavelets and multi-scale SSA, Phys. D, 142, 254-290, 2000.

Y ule, G. U., On a method of investigating periodicities in disturbed series, Philos. Trans. R. Soc. London, Ser. A, 226, 267-298, 1927.

Zebiak, S. E., and M. A. Cane, A model EI Niño-Southern O scillation, Mon. Weather Rev., 115, 2262-2278, 1987.

M. R. Allen, Space Science and Technology Department, Rutherford Appleton Laboratory, Chilton, Didcot OX11 0QX, UK.

M. D. Dettinger, U.S. Geological Survey, San Diego, CA 92123, USA.

M. Ghil, K. Ide, D. Kondrashov, A. Saunders, and F. Varadi, Department of Atmospheric Sciences, University of California, Los Angeles, Los Angeles, CA 90095-1565, USA. (ghil@atmos.ucla.edu)

M. E. Mann, Department of Environmental Sciences, University of Virginia, Charlottesville, VA 22903, USA.

A. W. Robertson, International Research Institute for Climate Prediction (IRI), P.O. Box 1000, Palisades, NY 109648000, USA.

Y. Tian, NASA Goddard Space Flight Center, Code 974, Greenbelt, MD 20771, USA.

P. Yiou, Laboratoire des Sciences du Climat et de I'Environnement, UMR CEA-CNRS, 91191 Gif-sur-Yvette, France. 